\title{
Syntheses and Structures of Functionalized
}

\section{[9]Cycloparaphenylenes as Carbon Nanohoops}

\author{
Bearing Carbomethoxy and \\ $N$-Phenylphthalimido Groups
}

Shuangjiang Li, Changfeng Huang, Haresh Thakellapalli, Behzad Farajidizaji,

Brian V. Popp, Jeffrey L. Petersen, and Kung K. Wang*

C. Eugene Bennett Department of Chemistry, West Virginia University

Morgantown, West Virginia 26506-6045

Supporting Information

Table of Contents

Page

S4 General Experimental Methods

S4 Experimental Procedure for Dibromide 3.

S5 Experimental Procedure for Cyclic Dimers syn-4 and anti-4 and Cyclic Trimers syn-5 and anti-5. 
Experimental Procedure for the Functionalized [9]CPP 6.

S8 Experimental Procedure for the Functionalized [9]CPP 8a.

S9 Experimental Procedure for the Functionalized [9]CPP 8b.

S10 Experimental Procedure for Diene 10.

S11 Experimental Procedure for Dibromide 11.

S12 Experimental Procedure for Cyclic Dimers syn-12 and anti-12 and Cyclic

Trimers syn-13 and anti-13.

S13 Experimental Procedure for the Functionalized [9]CPP 14.

S15 Figure S1. ORTEP Drawing of the Crystal Structure of 3.

S16-S23 Description of the X-ray Structural Analysis of $\mathbf{3}\left(\mathrm{C}_{22} \mathrm{H}_{18} \mathrm{Br}_{2} \mathrm{O}_{4}\right)$.

S24 Figure S2. ORTEP Drawing of the Crystal Structure of 14.

S25-S45 Description of the X-ray Structural Analysis of $\mathbf{1 4}$ $\left(\mathrm{C}_{78} \mathrm{H}_{54} \mathrm{O}_{12} \cdot 2 \mathrm{C}_{6} \mathrm{H}_{12} \cdot \mathrm{CH}_{2} \mathrm{Cl}_{2}\right)$.

S45 References Cited

S46-S47 $\quad{ }^{1} \mathrm{H}$ and ${ }^{13} \mathrm{C}$ NMR Spectra of Dibromide 3.

S48-S49 $\quad{ }^{1} \mathrm{H}$ and ${ }^{13} \mathrm{C}$ NMR Spectra of the Cyclic Dimers syn-4 and anti-4.

S50-S51 $\quad{ }^{1} \mathrm{H}$ and ${ }^{13} \mathrm{C}$ NMR Spectra of the Cyclic Dimers syn-4 or anti-4.

S52-S54 $\quad{ }^{1} \mathrm{H}$ and ${ }^{13} \mathrm{C}$ NMR Spectra of the Cyclic Trimer anti-5.

S55 $\quad{ }^{1}$ H NMR Spectrum of the Cyclic Trimer syn-5.

S56-S57 $\quad{ }^{1} \mathrm{H}$ and ${ }^{13} \mathrm{C}$ NMR Spectra of the Functionalized [9]CPP 6.

S58-S59 $\quad{ }^{1} \mathrm{H}$ and ${ }^{13} \mathrm{C}$ NMR Spectra of the Functionalized [9]CPP 8a.

S60-S61 $\quad{ }^{1} \mathrm{H}$ and ${ }^{13} \mathrm{C}$ NMR Spectra of the Functionalized [9]CPP $\mathbf{8 b}$.

S62-S63 $\quad{ }^{1} \mathrm{H}$ and ${ }^{13} \mathrm{C}$ NMR Spectra of Diene $\mathbf{1 0 .}$ 
S64-S65 $\quad{ }^{1} \mathrm{H}$ and ${ }^{13} \mathrm{C}$ NMR Spectra of Dibromide 11.

S66-S67 $\quad{ }^{1} \mathrm{H}$ and ${ }^{13} \mathrm{C}$ NMR Spectra of Cyclic Dimers syn-12 and anti-12.

S68-S69 $\quad{ }^{1} \mathrm{H}$ and ${ }^{13} \mathrm{C}$ NMR Spectra of the Cyclic Timer anti-13.

S70-S71 $\quad{ }^{1} \mathrm{H}$ and ${ }^{13} \mathrm{C}$ NMR Spectra of the Cyclic Timer syn-13.

S72-S73 $\quad{ }^{1} \mathrm{H}$ and ${ }^{13} \mathrm{C}$ NMR Spectra of the Functionalized [9]CPP 14.

S74-S75 UV-vis and Fluorescence Spectra of the Functionalized [9]CPPs $\mathbf{6}, \mathbf{8 a}, \mathbf{8 b}$, and 14. 


\section{General Experimental Methods}

All reactions were conducted in oven-dried $\left(120^{\circ} \mathrm{C}\right)$ glassware under an argon atmosphere. (E,E)-1,4-bis(4-bromophenyl)-1,3-butadiene ${ }^{9}$ and 1,8-bis(4-bromophenyl)1,7-octadiyne ${ }^{10}$ were prepared according to the reported procedures. Dimethyl acetylenedicarboxylate, $\mathrm{ZrCp}_{2} \mathrm{Cl}_{2}, \mathrm{Ni}(\operatorname{cod})_{2}, 2,2^{\prime}$-bipyridyl, and 2,3-dichloro-5,6-dicyano1,4-benzoquinone (DDQ), aniline, 1,4-diaminobenzene were purchased from chemical suppliers and were used as received. The UV-vis absorption spectrum was recorded on a Shimadzu UV-1800 spectrophotometer with a 1-nm resolution, and the baseline was corrected with a solvent filled square quartz cell. The fluorescence spectrum was recorded on a Shimadzu RF-5301PC spectrofluorophotometer with a 2-nm resolution.

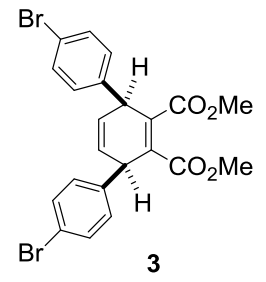

Experimental Procedure for Dibromide 3. To a mixture of (E,E)-1,4-bis(4bromophenyl)-1,3-butadiene (1.00 g, $2.74 \mathrm{mmol})$ and dimethyl acetylenedicarboxylate $(0.586 \mathrm{~g}, 4.12 \mathrm{mmol})$ was added dry toluene $(2 \mathrm{~mL})$ under argon in a sealed tube. The reaction mixture was stirred at $140{ }^{\circ} \mathrm{C}$ for $24 \mathrm{~h}$ before it was allowed to cool to rt. Toluene was removed in vacuo, and the residue was purified by flash column chromatography $($ silica gel/ethyl acetate:hexanes $=15: 85)$ to produce $3\left(1.18 \mathrm{~g}, 2.33 \mathrm{mmol}, 85 \%\right.$ yield, $R_{f}=$ $0.4)$ as a white solid: $\mathrm{mp}=120-121{ }^{\circ} \mathrm{C}$; IR $1724,1256 \mathrm{~cm}^{-1} ;{ }^{1} \mathrm{H}$ NMR $\left(400 \mathrm{MHz}, \mathrm{CDCl}_{3}\right)$ $\delta 7.47(4 \mathrm{H}, \mathrm{d}, J=8.6 \mathrm{~Hz}), 7.11(4 \mathrm{H}, \mathrm{d}, J=8.2 \mathrm{~Hz}), 5.76(2 \mathrm{H}, \mathrm{d}, J=2.0 \mathrm{~Hz}), 4.43(2 \mathrm{H}$, d, $J=2.0 \mathrm{~Hz}), 3.59(6 \mathrm{H}, \mathrm{s}) ;{ }^{13} \mathrm{C} \mathrm{NMR}\left(\mathrm{CDCl}_{3}, 100 \mathrm{MHz}\right) \delta 167.48,139.96,135.47,131.88$, 
129.85, 125.96, 121.20, 52.18, 43.33; HRMS (ESI) calcd for $\mathrm{C}_{22} \mathrm{H}_{18} \mathrm{Br}_{2} \mathrm{O}_{4}\left(\mathrm{M}^{+}\right)$504.9645, 506.9624, 508.9604 found 504.9650, 506.9629, 508.9609.

Recrystallization of $\mathbf{3}$ from a mixture of dichloromethane and hexanes produced a single crystal suitable for X-ray structure analysis.
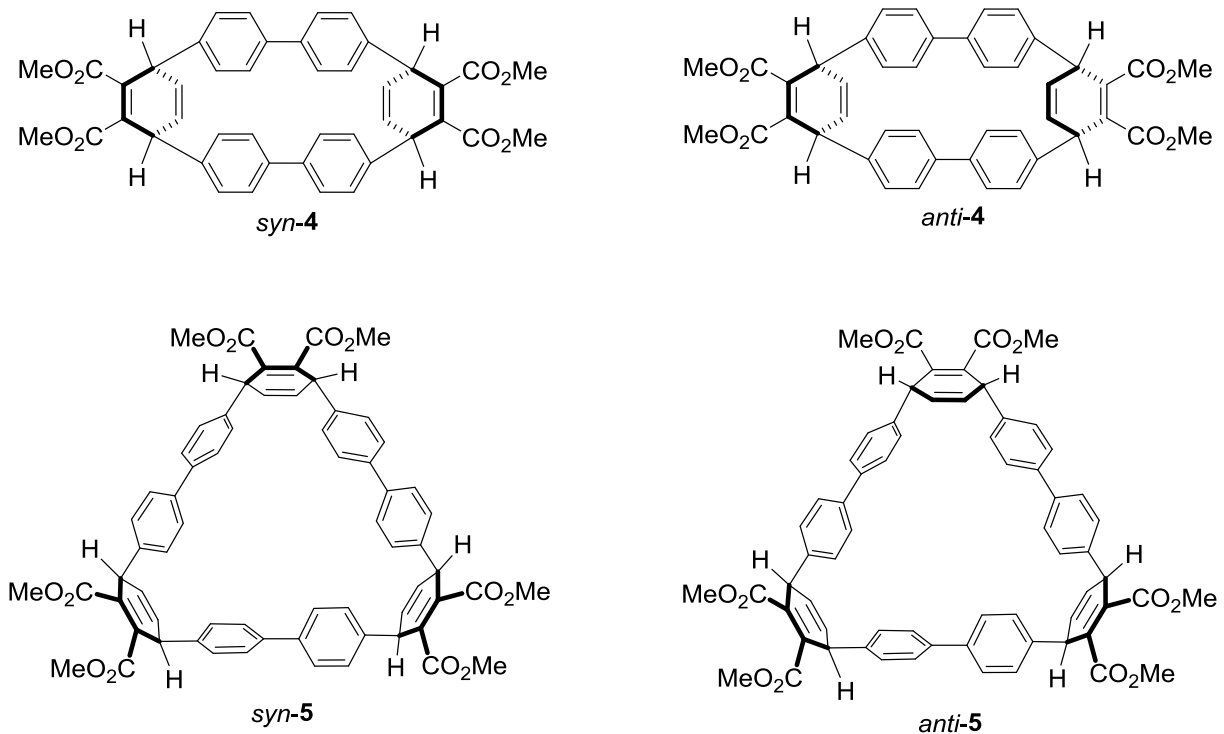

\section{Experimental Procedure for Cyclic Dimers syn-4 and anti-4 and Cyclic Trimers syn-}

5 and anti-5. To a $200 \mathrm{~mL}$-flask were added $0.500 \mathrm{~g}$ of $3(0.988 \mathrm{mmol})$ and $0.370 \mathrm{~g}$ of 2,2'- bipyridyl $(2.37 \mathrm{mmol})$. The flask was flushed with nitrogen and placed in a glovebox under a nitrogen atmosphere before $0.650 \mathrm{~g}$ of $\mathrm{Ni}(\mathrm{cod})_{2}(2.36 \mathrm{mmol})$ was added. The flask was fitted with a condenser and a rubber septum and then removed from the glovebox before $100 \mathrm{~mL}$ of THF was introduced via cannula. The reaction mixture was heated at reflux for $18 \mathrm{~h}$ before it was allowed to cool to $\mathrm{rt}$. Then the reaction mixture was passed through a short pad of silica gel column $(4 \mathrm{~cm})$ and eluted with a mixture of ethyl acetate and dichloromethane. The combined eluates were concentrated, and the residue was purified by flash column chromatography (silica gel/dichloromethane:diethyl ether = 97:3 
to $90: 10)$ to produce fractions containing a mixture of $s y n-\mathbf{4}$ and anti-4, fractions containing mainly pure anti-5, and fractions containing syn-5 with other unidentified materials. The ratio between $\mathbf{4}$ and $\mathbf{5}$ is estimated by NMR spectroscopy to be approximately 1:10. Further purification by preparative TLC (ethyl acetate:hexanes $=3: 7$ ) allowed the isolation of relatively pure samples of syn-4, anti-4, and anti-5 and a sample of syn-5 with unidentified impurities for structural elucidation. syn-4 and anti-4 (sample 1, one major and one minor, $R_{f}=0.56$, dichloromethane: diethyl ether $\left.=95: 5\right):$ IR 1716, $1272 \mathrm{~cm}^{-1} ;{ }^{1} \mathrm{H} \mathrm{NMR}\left(\mathrm{CDCl}_{3}\right.$, $600 \mathrm{MHz}) \delta 6.85(8 \mathrm{H}, \mathrm{d}, J=7.6 \mathrm{~Hz}), 6.78(4 \mathrm{H}, \mathrm{d}, J=8.2 \mathrm{~Hz}), 6.67(4 \mathrm{H}, \mathrm{dd}, J=4.1,2.9$ $\mathrm{Hz}), 4.69(4 \mathrm{H}, \mathrm{t}, J=3.5 \mathrm{~Hz}), 3.90(12 \mathrm{H}, \mathrm{s}) ;{ }^{13} \mathrm{C} \mathrm{NMR}\left(\mathrm{CDCl}_{3}, 150 \mathrm{MHz}\right) \delta 168.42,141.63$, 138.97, 138.27, 131.99, 127.35, 126.34, 52.68, 41.78; HRMS (ESI) calcd for $\mathrm{C}_{44} \mathrm{H}_{36} \mathrm{O}_{8}$ $\left(\mathrm{M}^{+}\right)$692.2405, found 692.2419. syn-4 or anti-4 (sample 2, $R_{f}=0.52$, dichloromethane:diethyl ether $=95: 5): \mathrm{mp}>250{ }^{\circ} \mathrm{C}$; IR 1718, $1260 \mathrm{~cm}^{-1} ;{ }^{1} \mathrm{H} \mathrm{NMR}\left(\mathrm{CDCl}_{3}\right.$, $600 \mathrm{MHz}) \delta 6.85(8 \mathrm{H}, \mathrm{d}, J=8.2 \mathrm{~Hz}), 6.78(8 \mathrm{H}, \mathrm{d}, J=8.2 \mathrm{~Hz}), 6.65(4 \mathrm{H}, \mathrm{dd}, J=4.4,2.6$ $\mathrm{Hz}), 4.69(4 \mathrm{H}, \mathrm{t}, J=3.5 \mathrm{~Hz}), 3.91(12 \mathrm{H}, \mathrm{s}) ;{ }^{13} \mathrm{C} \mathrm{NMR}\left(\mathrm{CDCl}_{3}, 150 \mathrm{MHz}\right) \delta 168.42,141.62$, 138.96, 138.31, 131.99, 127.36, 126.35, 52.70, 41.80; HRMS (ESI) calcd for $\mathrm{C}_{44} \mathrm{H}_{36} \mathrm{O}_{8}$ $\left(\mathrm{M}^{+}\right)$692.2405, found 692.2413. anti-5 $\left(R_{f}=0.39\right.$, dichloromethane:diethyl ether = 95:5): IR 1718, $1250 \mathrm{~cm}^{-1} ;{ }^{1} \mathrm{H}$ NMR $\left(\mathrm{C}_{6} \mathrm{D}_{6}, 400 \mathrm{MHz}\right) \delta 7.51-7.57(12 \mathrm{H}, \mathrm{m}), 7.38(12 \mathrm{H}, \mathrm{d}, J=$ 8.2 Hz), 5.57-5.68 (6 H, m), 4.50-4.57 (6 H, m), $3.31(6 \mathrm{H}, \mathrm{s}), 3.28(6 \mathrm{H}, \mathrm{s}), 3.27$ (6 H, s); ${ }^{1} \mathrm{H} \mathrm{NMR}\left(\mathrm{CDCl}_{3}, 400 \mathrm{MHz}\right) \delta 7.63-7.68(12 \mathrm{H}, \mathrm{m}), 7.34-7.38(12 \mathrm{H}, \mathrm{m}), 5.86-5.96(6 \mathrm{H}$, m), 4.55-4.60 (6 H, m), $3.66(6 \mathrm{H}, \mathrm{s}), 3.647(6 \mathrm{H}, \mathrm{s}), 3.645(6 \mathrm{H}, \mathrm{s}) ;{ }^{13} \mathrm{C} \mathrm{NMR}\left(\mathrm{CDCl}_{3}, 100\right.$ MHz) $\delta 168.16,167.95,167.74,140.22,139.99,139.80,139.07,139.01,138.90,136.95$, $136.00,135.09,128.71,128.64,128.53,126.96,126.77,126.42,126.04,52.20,52.17$, 43.69, 43.52, 43.41; HRMS (ESI) calcd for $\mathrm{C}_{66} \mathrm{H}_{55} \mathrm{O}_{12}\left(\mathrm{MH}^{+}\right)$1039.3688, found 1039.3684. 
$s y n-5$ (sample not fully purified, $R_{f}=0.22$, dichloromethane:diethyl ether $=95: 5$ ): IR 1724, $1253 \mathrm{~cm}^{-1} ;{ }^{1} \mathrm{H} \mathrm{NMR}\left(\mathrm{CDCl}_{3}, 400 \mathrm{MHz}\right) \delta 7.66(12 \mathrm{H}, \mathrm{d}, J=8.2 \mathrm{~Hz}), 7.36(12 \mathrm{H}, \mathrm{d}, J=$ $8.6 \mathrm{~Hz}), 5.89(6 \mathrm{H}, \mathrm{d}, J=2.8 \mathrm{~Hz}), 4.57(6 \mathrm{H}, \mathrm{d}, J=2.8 \mathrm{~Hz}), 3.66(18 \mathrm{H}, \mathrm{s})$.

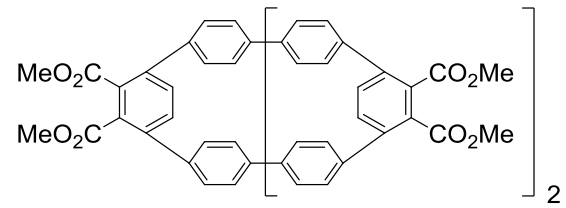

Experimental Procedure for the Functionalized [9]CPP 6. To a 10-mL flask were added $0.010 \mathrm{~g}(0.0097 \mathrm{mmol})$ of anti-5 and $0.030 \mathrm{~g}(0.13 \mathrm{mmol})$ of DDQ. The flask was flushed with argon, and then $2 \mathrm{~mL}$ of chlorobenzene was introduced by using a syringe. The reaction mixture was heated at $120{ }^{\circ} \mathrm{C}$ for $2 \mathrm{~h}$ before it was allowed to cool to $\mathrm{rt}$. Dichloromethane $(50 \mathrm{~mL})$ was added, and the solution was passed through a basic aluminum oxide column ( $4 \mathrm{~cm}$ high, $2.5 \mathrm{~cm}$ in diameter). The column was eluted with an additional $200 \mathrm{~mL}$ of a mixture of dichloromethane and ethyl acetate (1:1). The combined eluates were concentrated in vacuo to afford $6(0.0081 \mathrm{~g}, 0.0078 \mathrm{mmol}, 80 \%$ yield $)$ as a white solid.

Treatment of the sample containing syn-5 with DDQ also produced the fully aromatized 6.

In a separated experiment, the crude cyclic products containing a mixture of $\mathbf{4}$ and 5, produced from $0.500 \mathrm{~g}(0.988 \mathrm{mmol})$ of $\mathbf{3}$, were not further purified and were treated directly with DDQ $(0.300 \mathrm{~g}, 1.32 \mathrm{mmol})$ in $5 \mathrm{~mL}$ of chlorobenzene at $120{ }^{\circ} \mathrm{C}$ for $3 \mathrm{~h}$. Dichloromethane $(50 \mathrm{~mL})$ was added, and the reaction mixture was passed through a basic aluminum oxide column ( $4 \mathrm{~cm}$ high, $2.5 \mathrm{~cm}$ in diameter). The column was eluted with an 
additional $100 \mathrm{~mL}$ of a mixture of dichloromethane and ethyl acetate (1:1). The combined eluates were concentrated in vacuo, and the residue was purified by flash column chromatography (silica gel/ethyl acetate:methylene chloride $=3: 97, R_{f}=0.15$ ) to produce $0.049 \mathrm{~g}(0.047 \mathrm{mmol}, 14 \%$ yield over two steps $)$ of 6 : IR $1737,1236 \mathrm{~cm}^{-1} ;{ }^{1} \mathrm{H} \mathrm{NMR}$ $\left(\mathrm{CDCl}_{3}, 400 \mathrm{MHz}\right) \delta 7.54(12 \mathrm{H}, \mathrm{d}, J=8.6 \mathrm{~Hz}), 7.43(12 \mathrm{H}, \mathrm{d}, J=8.6 \mathrm{~Hz}), 6.88(6 \mathrm{H}, \mathrm{s})$, $3.85(18 \mathrm{H}, \mathrm{s}) ;{ }^{13} \mathrm{C} \mathrm{NMR}\left(\mathrm{CDCl}_{3}, 150 \mathrm{MHz}\right) \delta 168.15,139.67,139.08,138.80,135.38$, 129.80, 129.64, 127.15, 52.75; HRMS (ESI) calcd for $\mathrm{C}_{66} \mathrm{H}_{49} \mathrm{O}_{12}\left(\mathrm{MH}^{+}\right)$1033.3219, found 1033.3220.

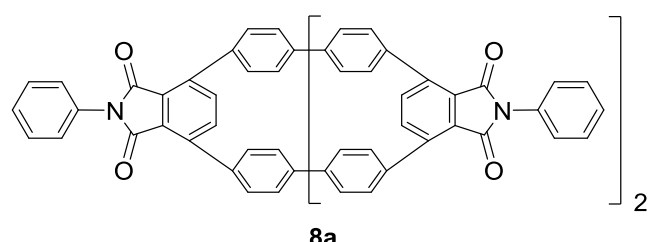

Experimental Procedure for the Functionalized [9]CPP 8a. A mixture of $0.0070 \mathrm{~g}(6.8$ $\left.\times 10^{-3} \mathrm{mmol}\right)$ of $6,0.1 \mathrm{~mL}$ of triethylamine, $0.03 \mathrm{~mL}(0.33 \mathrm{mmol})$ of aniline and $3 \mathrm{~mL}$ of pyridine in a sealed tube under an argon atmosphere was heated at $150{ }^{\circ} \mathrm{C}$ for $40 \mathrm{~h}$ before it was allowed to cool to rt. The reaction was concentrated in vacuo, and the residue was purified by preparative TLC to give $8 \mathbf{a}\left(0.0050 \mathrm{~g}, 4.5 \times 10^{-3} \mathrm{mmol}, 66 \%\right.$ yield $)$ as a bright yellow solid: IR 1716, $1374 \mathrm{~cm}^{-1} ;{ }^{1} \mathrm{H}$ NMR $\left(\mathrm{CD}_{2} \mathrm{Cl}_{2}, 600 \mathrm{MHz}\right) \delta 7.68(12 \mathrm{H}, \mathrm{d}, J=8.8$ $\mathrm{Hz}), 7.64(12 \mathrm{H}, \mathrm{d}, J=8.8 \mathrm{~Hz}), 7.57(6 \mathrm{H}, \mathrm{t}, J=7.6 \mathrm{~Hz}), 7.52(6 \mathrm{H}, \mathrm{dd}, J=8.8,1.2 \mathrm{~Hz})$, $7.46(3 \mathrm{H}, \mathrm{td}, J=7.3,1.2 \mathrm{~Hz}), 7.24(6 \mathrm{H}, \mathrm{s}) ;{ }^{13} \mathrm{C} \mathrm{NMR}\left(\mathrm{CD}_{2} \mathrm{Cl}_{2}, 150 \mathrm{MHz}\right) \delta 167.33,139.87$, 138.67, 138.27, 136.72, 132.54, 130.36, 129.61, 128.72, 128.44, 127.69, 127.56; HRMS (ESI) calcd for $\mathrm{C}_{78} \mathrm{H}_{46} \mathrm{~N}_{3} \mathrm{O}_{6}\left(\mathrm{MH}^{+}\right) 1120.3381$, found 1120.3418 . 


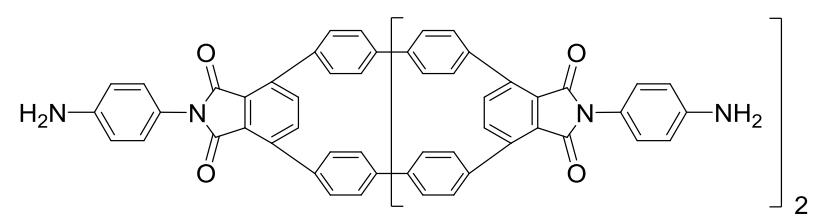

$8 b$

Experimental Procedure for the Functionalized [9]CPP 8b. A mixture of $0.0090 \mathrm{~g}$ of $6(0.0087 \mathrm{mmol}), 0.1 \mathrm{~mL}$ of triethylamine, $0.050 \mathrm{~g}$ of 1,4-diaminobenzene $(0.46 \mathrm{mmol})$ and $3 \mathrm{~mL}$ of pyridine in a sealed tube under an argon atmosphere was heated at $150{ }^{\circ} \mathrm{C}$ for $48 \mathrm{~h}$ before it was allowed to cool to rt. The reaction mixture was concentrated in vacuo, and the residue was washed with methanol and then passed through a short pad of silica gel using $100 \mathrm{~mL}$ of ethyl acetate as eluent. The combined eluates were concentrated in vacuo, and the residue was washed with methanol and pentane. After $2 \mathrm{~h}$ under high vacuum, $8 \mathbf{b}$ (0.0050 g, 53\% yield) was obtained as a yellow solid: IR 3375, 1711, 1517 $\mathrm{cm}^{-1} ;{ }^{1} \mathrm{H}$ NMR (DMSO- $\left.d_{6}, 600 \mathrm{MHz}\right) \delta 7.78(12 \mathrm{H}, \mathrm{d}, J=8.2 \mathrm{~Hz}), 7.72(12 \mathrm{H}, \mathrm{d}, J=8.2$ $\mathrm{Hz}), 7.38(6 \mathrm{H}, \mathrm{s}), 7.11(6 \mathrm{H}, \mathrm{d}, J=8.8 \mathrm{~Hz}), 6.66(6 \mathrm{H}, \mathrm{d}, J=8.8 \mathrm{~Hz}), 5.33(6 \mathrm{H}, \mathrm{s}) ;{ }^{13} \mathrm{C}$ NMR (DMSO- $\left.d_{6}, 150 \mathrm{MHz}\right) \delta 167.07,148.70,138.35,137.45,136.18,135.86,129.89$, $128.42,128.32,126.83,119.85,113.43$; HRMS (ESI) calcd for $\mathrm{C}_{78} \mathrm{H}_{49} \mathrm{~N}_{6} \mathrm{O}_{6}\left(\mathrm{MH}^{+}\right)$ 1165.3708, found 1165.3777. 


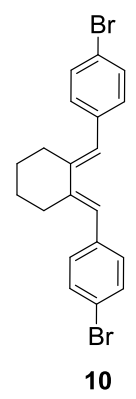

Experimental Procedure for Diene 10. ${ }^{10 \mathrm{a}, \mathrm{c}}$ To a solution of $\mathrm{Cp}_{2} \mathrm{ZrCl}_{2}(2.55 \mathrm{~g}, 8.74 \mathrm{mmol})$ in THF $(30 \mathrm{~mL})$ at $-78^{\circ} \mathrm{C}$ under an argon atmosphere was added $n$-butyllithium $(7.00 \mathrm{~mL}$ of a $2.5 \mathrm{M}$ solution in hexanes, $17.5 \mathrm{mmol})$. After $1 \mathrm{~h}$ at $-78^{\circ} \mathrm{C}, 1,8$-bis(4-bromophenyl)1,7-octadiyne $(3.03 \mathrm{~g}, 7.28 \mathrm{mmol})$ in $10 \mathrm{~mL}$ of THF under argon was added slowly. The reaction mixture was then allowed to warm to rt. After $12 \mathrm{~h}$, the reaction mixture was poured into $300 \mathrm{~mL}$ of a $3 \mathrm{M}$ aqueous $\mathrm{HCl}$ solution. After $30 \mathrm{~min}$ of stirring, the reaction mixture was extracted with dichloromethane $(2 \times 100 \mathrm{~mL})$. The combined organic layers were washed with a saturated $\mathrm{Na}_{2} \mathrm{CO}_{3}$ solution and dried over $\mathrm{MgSO}_{4}$. After passing through a short silica gel column, the solvent was evaporated in vacuo, and the residue was washed with cold hexanes to give $\mathbf{1 0}(2.50 \mathrm{~g}, 5.98 \mathrm{mmol}, 82 \%$ yield $)$ as a white solid: IR 2930, 1485, $1009 \mathrm{~cm}^{-1} ;{ }^{1} \mathrm{H} \mathrm{NMR}\left(\mathrm{CDCl}_{3}, 400 \mathrm{MHz}\right) \delta 7.46(4 \mathrm{H}, \mathrm{d}, J=8.2 \mathrm{~Hz}), 7.15$ (4 $\mathrm{H}, \mathrm{d}, J=8.6 \mathrm{~Hz}), 6.52(2 \mathrm{H}, \mathrm{s}), 2.56(4 \mathrm{H}, \mathrm{m}), 1.65(4 \mathrm{H}, \mathrm{m}) ;{ }^{13} \mathrm{C} \mathrm{NMR}\left(\mathrm{CDCl}_{3}, 100 \mathrm{MHz}\right)$ $\delta$ 145.02, 136.69, 131.19, 131.03, 123.22, 120.28, 29.91, 26.10; HRMS (ESI) calcd for $\mathrm{C}_{20} \mathrm{H}_{18} \mathrm{Br}_{2}\left(\mathrm{M}^{+}\right)$415.9770, 417.9749, 419.9729, found 415.9788, 417.9767, 419.9742. 


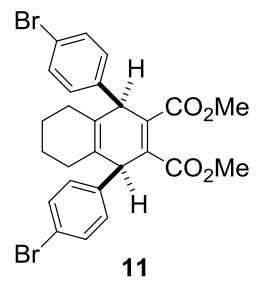

Experimental Procedure for Dibromide 11. To a mixture of diene 10 (1.70 g, $4.07 \mathrm{mmol})$ and dimethyl acetylenedicarboxylate $(0.867 \mathrm{~g}, 6.10 \mathrm{mmol})$ was added $2 \mathrm{~mL}$ of dry toluene under argon. The reaction mixture was stirred at $100{ }^{\circ} \mathrm{C}$ for $20 \mathrm{~h}$ before it was allowed to cool to rt. Toluene was evaporated in vacuo, and the residue was purified by flash column chromatography (silica gel/ethyl acetate:hexanes $=15: 85)$ to afford $11(2.00 \mathrm{~g}, 3.57 \mathrm{mmol}$, $88 \%$ yield) as a white solid: $\mathrm{mp} 142-143{ }^{\circ} \mathrm{C}$; IR $1721,1259 \mathrm{~cm}^{-1}$; ${ }^{1} \mathrm{H}$ NMR (400 MHz, $\left.\mathrm{CDCl}_{3}\right) \delta 7.44(4 \mathrm{H}, \mathrm{d}, J=8.2 \mathrm{~Hz}), 7.11(4 \mathrm{H}, \mathrm{d}, J=8.6 \mathrm{~Hz}), 4.18(2 \mathrm{H}, \mathrm{s}), 1.84(4 \mathrm{H}, \mathrm{t}, J$ $=6.1 \mathrm{~Hz}), 1.55-1.65(2 \mathrm{H}, \mathrm{m}), 1.40-1.50(2 \mathrm{H}, \mathrm{m}) ;{ }^{13} \mathrm{C} \mathrm{NMR}\left(\mathrm{CDCl}_{3}, 100 \mathrm{MHz}\right) \delta 167.63$, 139.52, 135.42, 131.62, 130.45, 128.29, 121.08, 52.11, 48.56, 27.96, 22.49; HRMS (ESI) calcd for $\mathrm{C}_{26} \mathrm{H}_{25} \mathrm{Br}_{2} \mathrm{O}_{4}\left(\mathrm{MH}^{+}\right)$559.0114, 561.0094, 563.0073, found 559.0120, 561.0100, 563.0078. 

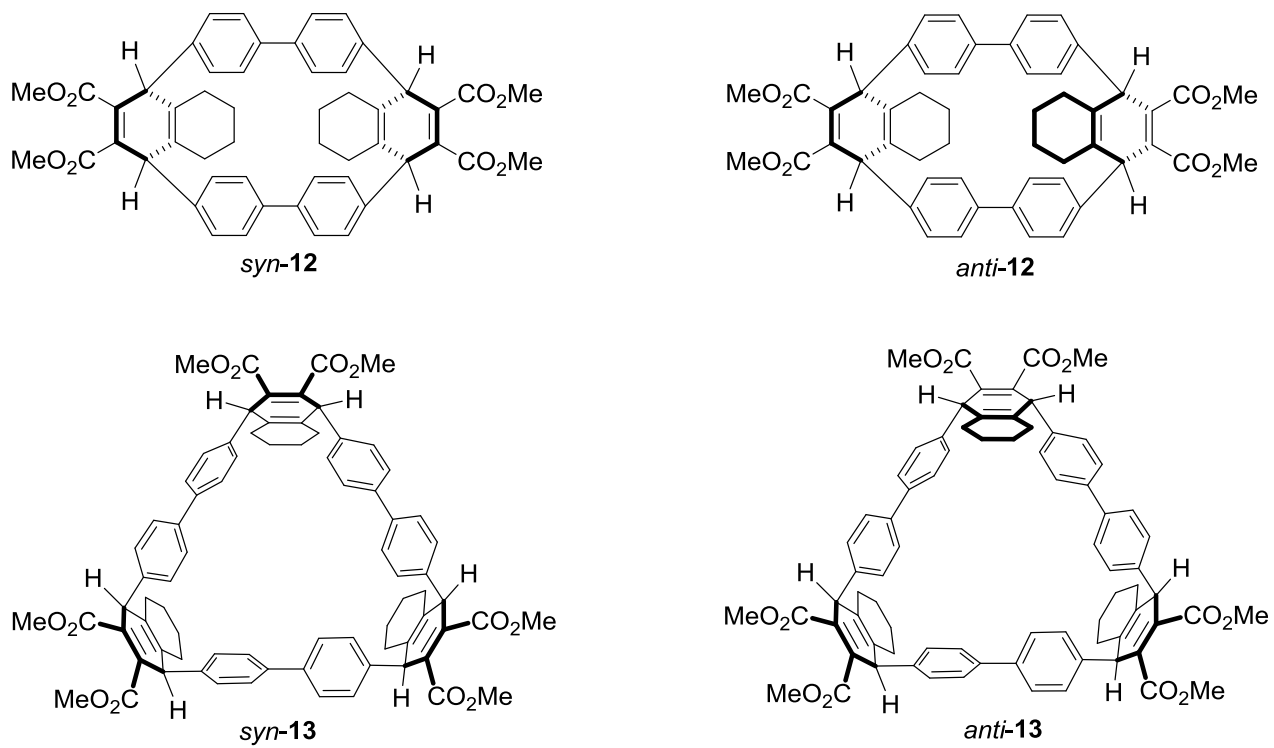

\section{Experimental Procedure for Cyclic Dimers syn-12 and anti-12 and Cyclic Trimers}

syn-13 and anti-13. To a $250 \mathrm{~mL}-$ flask were added $1.00 \mathrm{~g}$ of $\mathbf{1 1}(1.79 \mathrm{mmol})$ and $0.700 \mathrm{~g}$ of 2,2'-bipyridyl (4.48 mmol). The flask was flushed with nitrogen and placed in a glovebox under a nitrogen atmosphere before $1.20 \mathrm{~g}$ of Ni(cod $)_{2}(4.36 \mathrm{mmol})$ was added. The flask was fitted with a condenser and a rubber septum and then removed from the glovebox before $150 \mathrm{~mL}$ of THF was introduced via cannula. The reaction mixture was heated at reflux for $18 \mathrm{~h}$ before it was allowed to cool to rt. Then the reaction mixture was passed through a short silica gel column $(4 \mathrm{~cm})$ and eluted with a mixture of ethyl acetate and dichloromethane. The combined eluates were concentrated, and the residue was purified by flash column chromatography (silica gel/ethyl acetate:hexanes $=40: 60$ to 60:40) to produce a small amount (ca. 3\%) of a mixture of syn-12 and anti-12 as a white solid, $0.205 \mathrm{~g}$ of anti-13 $(0.171 \mathrm{mmol}, 29 \%$ yield $)$ as a white solid, and an impure sample of syn13. Further purification of $s y n-\mathbf{1 3}$ by preparative TLC afforded $0.051 \mathrm{~g}(0.042 \mathrm{mmol}, 7 \%$ yield) of a pure sample of $\operatorname{syn}-\mathbf{1 3}$ as a white solid. syn-12 and anti-12: $\mathrm{mp}>330{ }^{\circ} \mathrm{C}$; IR $1717,1259 \mathrm{~cm}^{-1} ;{ }^{1} \mathrm{H} \mathrm{NMR}\left(\mathrm{CDCl}_{3}, 600 \mathrm{MHz}\right) \delta 6.80(8 \mathrm{H}, \mathrm{d}, J=8.2 \mathrm{~Hz}), 6.751$ and 6.749 
( $8 \mathrm{H}$, two doublets from the two isomers, $J=8.2 \mathrm{~Hz}), 4.35(4 \mathrm{H}, \mathrm{s}), 3.90(12 \mathrm{H}, \mathrm{s})$, 2.58-2.61 (4 H, m), 2.31-2.35 (4 H, m), 1.85-1.94 (4 H, m), 1.77-1.85 (4 H, m); ${ }^{13} \mathrm{C} \mathrm{NMR}$ $\left(\mathrm{CDCl}_{3}, 150 \mathrm{MHz}\right) \delta 168.58,142.42,142.39,138.98,137.79,137.78,135.47,135.45$, 127.20, 126.36, 52.62, 47.86, 31.54, 22.84, 22.82; HRMS (ESI) calcd for $\mathrm{C}_{52} \mathrm{H}_{48} \mathrm{O}_{8}\left(\mathrm{M}^{+}\right)$ 800.3344, found 800.3364. anti-13: IR 1723, $1261 \mathrm{~cm}^{-1} ;{ }^{1} \mathrm{H} \mathrm{NMR}\left(\mathrm{CDCl}_{3}, 600 \mathrm{MHz}\right) \delta$ 7.67-7.71 (12 H, m), $7.37(12 \mathrm{H}, \mathrm{d}, J=8.2 \mathrm{~Hz}), 4.31(6 \mathrm{H}, \mathrm{s}), 3.63(6 \mathrm{H}, \mathrm{s}), 3.619(6 \mathrm{H}$, s), $3.617(6 \mathrm{H}, \mathrm{s}), 1.91-2.06(12 \mathrm{H}, \mathrm{m}), 1.57-1.67(6 \mathrm{H}, \mathrm{m}), 1.45-1.56(6 \mathrm{H}, \mathrm{m}) ;{ }^{13} \mathrm{C} \mathrm{NMR}$ $\left(\mathrm{CDCl}_{3}, 150 \mathrm{MHz}\right) \delta 168.11,168.09,139.71,138.57,138.51,135.92,135.88,135.83$, 129.29, 128.66, 128.62, 126.40, 126.36, 52.07, 52.04, 48.84, 28.00, 22.62; HRMS (ESI) calcd for $\mathrm{C}_{78} \mathrm{H}_{73} \mathrm{O}_{12}\left(\mathrm{MH}^{+}\right)$1201.5097, found 1201.5098. syn-13: IR 1725, $1262 \mathrm{~cm}^{-1} ;{ }^{1} \mathrm{H}$ $\operatorname{NMR}\left(\mathrm{CDCl}_{3}, 600 \mathrm{MHz}\right) \delta 7.69(12 \mathrm{H}, \mathrm{d}, J=8.8 \mathrm{~Hz}), 7.37(12 \mathrm{H}, \mathrm{d}, J=8.2 \mathrm{~Hz}), 4.31(6$ $\mathrm{H}, \mathrm{s}), 3.63(18 \mathrm{H}, \mathrm{s}), 1.91-2.04(12 \mathrm{H}, \mathrm{m}), 1.57-1.65(6 \mathrm{H}, \mathrm{m}), 1.44-1.52(6 \mathrm{H}, \mathrm{m}) ;{ }^{13} \mathrm{C}$ $\operatorname{NMR}\left(\mathrm{CDCl}_{3}, 100 \mathrm{MHz}\right) \delta 168.08,139.73,138.50,135.87,129.29,128.63,126.36,52.08$, 48.86, 28.01, 22.63; HRMS (ESI) calcd for $\mathrm{C}_{78} \mathrm{H}_{73} \mathrm{O}_{12}\left(\mathrm{MH}^{+}\right)$1201.5097, found 1201.5104.

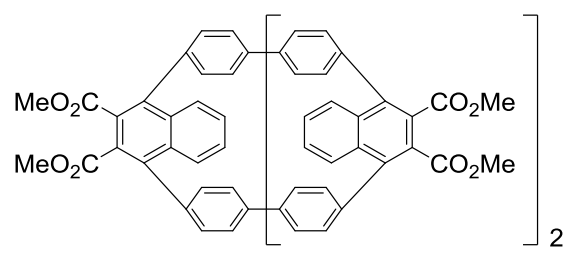

14

Experimental Procedure for the Functionalized [9]CPP 14. To a 20-mL flask were added $0.160 \mathrm{~g}(0.133 \mathrm{mmol})$ of anti-13 and $0.450 \mathrm{~g}(1.98 \mathrm{mmol})$ of DDQ. The flask was flushed with argon and then $6 \mathrm{~mL}$ of chlorobenzene was introduced by using a syringe. The reaction mixture was heated at $120^{\circ} \mathrm{C}$ for $15 \mathrm{~h}$ before it was allowed to cool to $\mathrm{rt}$. Dichloromethane $(10 \mathrm{~mL})$ was added and the solution was passed through a basic 
aluminum oxide column ( $4 \mathrm{~cm}$ high, $2.5 \mathrm{~cm}$ in diameter). The column was eluted with an additional $100 \mathrm{~mL}$ of dichloromethane and ethyl acetate (1:1). The combined eluates were concentrated in vacuo to afford $14(0.129 \mathrm{~g}, 0.109 \mathrm{mmol}, 82 \%$ yield $)$ as a white solid: IR 1736, $1221 \mathrm{~cm}^{-1} ;{ }^{1} \mathrm{H} \mathrm{NMR}\left(\mathrm{CDCl}_{3}, 400 \mathrm{MHz}\right) \delta 8.27(6 \mathrm{H}, \mathrm{dd}, J=6.5,3.3 \mathrm{~Hz}), 7.64(6 \mathrm{H}$, $\mathrm{dd}, J=6.4,3.3 \mathrm{~Hz}), 7.48(12 \mathrm{H}, \mathrm{d}, J=8.2 \mathrm{~Hz}), 7.33(12 \mathrm{H}, \mathrm{d}, J=8.6 \mathrm{~Hz}), 3.12(18 \mathrm{H}, \mathrm{s})$; ${ }^{13} \mathrm{C} \mathrm{NMR}\left(\mathrm{CDCl}_{3}, 100 \mathrm{MHz}\right) \delta 168.57,140.55,136.50,136.28,132.12,131.20,131.05$, 127.73, 127.60, 127.18, 52.16; HRMS (ESI) calcd for $\mathrm{C}_{78} \mathrm{H}_{55} \mathrm{O}_{12}\left(\mathrm{MH}^{+}\right)$1183.3688, found 1183.3692.

Treatment of syn-13 with DDQ also produced the fully aromatized $\mathbf{1 4 .}$

Recrystallization of $\mathbf{1 4}$ from a mixture of dichloromethane and cyclohexane produced a single crystal suitable for X-ray structure analysis. 


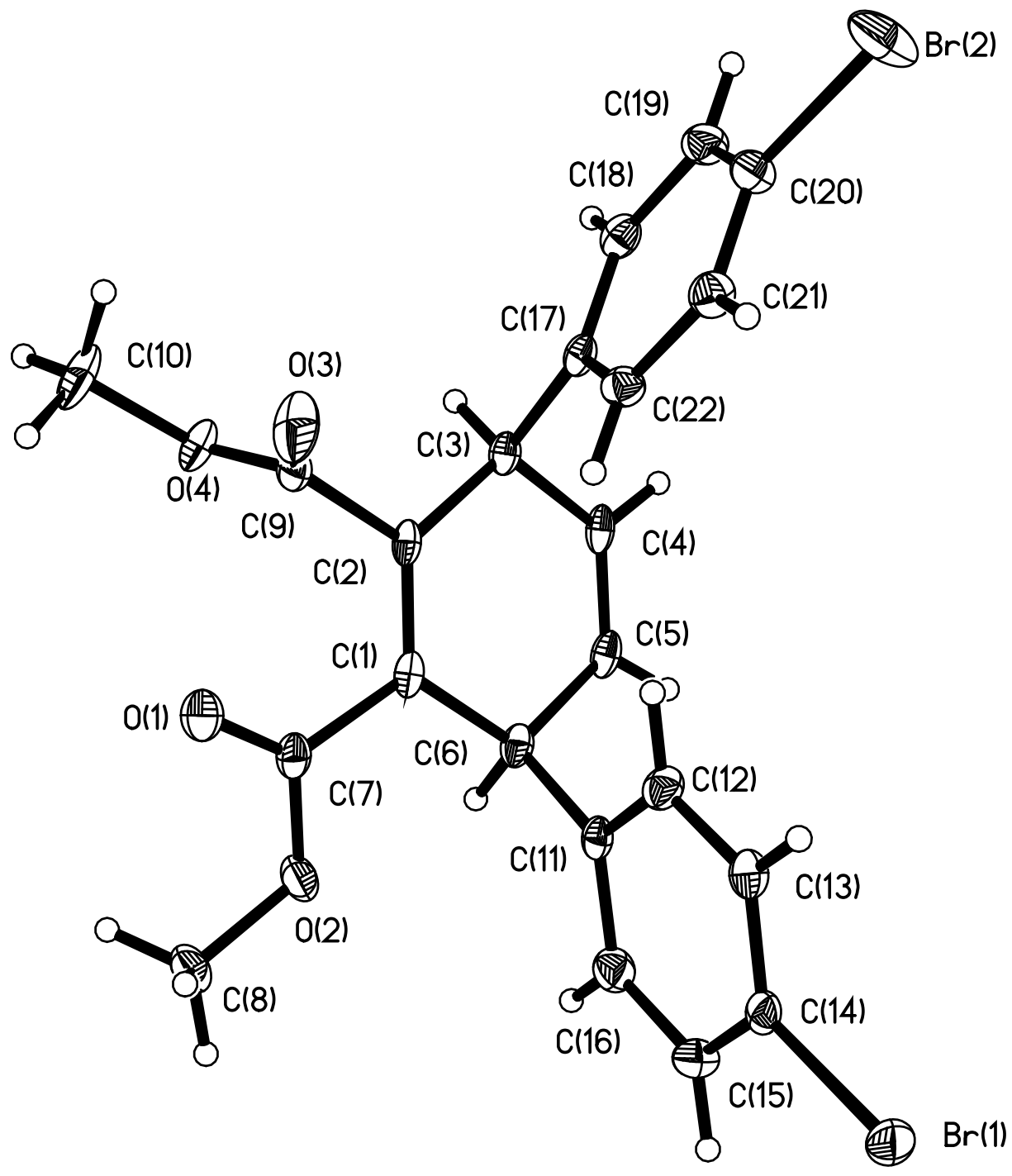

Figure S1. ORTEP Drawing of the Crystal Structure of 3.

Perspective view of the molecular structure of $\mathbf{3}\left(\mathrm{C}_{22} \mathrm{H}_{18} \mathrm{Br}_{2} \mathrm{O}_{4}\right)$ with the atom labeling scheme for the independent non-hydrogen atoms. The thermal ellipsoids are scaled to enclose $50 \%$ probability. 


\section{Description of the X-ray Structural Analysis of $3\left(\mathrm{C}_{22} \mathrm{H}_{18} \mathrm{Br}_{2} \mathrm{O}_{4}\right)$}

A colorless crystal of $\mathbf{3}\left(\mathrm{C}_{22} \mathrm{H}_{18} \mathrm{Br}_{2} \mathrm{O}_{4}\right)$ was covered in a polybutene oil (SigmaAldrich) and placed on the end of a MiTeGen loop. The sample was cooled to $105 \mathrm{~K}$ with an Oxford Cryostream 700 system and optically aligned on a Bruker AXS D8 Venture fixed-chi X-ray diffractometer equipped with a Triumph monochromator, a Mo Ka radiation source $(\lambda=0.71073 \AA)$, and a PHOTON 100 CMOS detector. Two sets of 12 frames each were collected using the omega scan method with a $10 \mathrm{~s}$ exposure time. Integration of these frames followed by reflection indexing and least-squares refinement produced a crystal orientation matrix for the monoclinic crystal lattice.

Data collection consisted of the measurement of a total of 372 frames in two runs using omega scans with the detector held at $5.00 \mathrm{~cm}$ from the crystal. Frame scan parameters are summarized in Table 1 below:

Table 1. Data collection details for $3\left(\mathrm{C}_{22} \mathrm{H}_{18} \mathrm{Br}_{2} \mathrm{O}_{4}\right)$.

$\begin{array}{cccccccc}\text { Run } & 2 \theta & \omega & \varphi & \chi & \text { Scan Width }\left(^{\circ}\right) & \text { Frames } & \begin{array}{c}\text { Exposure Time } \\ (\mathrm{sec})\end{array} \\ 1 & 22.76 & -160.24 & -69.76 & 54.79 & 1.00 & 186 & 10.00 \\ 2 & 22.76 & -160.24 & -179.21 & 54.79 & 1.00 & 186 & 10.00\end{array}$

The APEX2 software program (version 2014.1-7) ${ }^{\mathrm{S} 1}$ was used for diffractometer control, preliminary frame scans, indexing, orientation matrix calculations, least-squares refinement of cell parameters, and the data collection. The frames were integrated with the Bruker SAINT software package using a narrow-frame algorithm. The integration of the data using a monoclinic unit cell yielded a total of 21353 reflections to a maximum $\theta$ angle 
of $30.00^{\circ}(0.71 \AA ̊$ resolution), of which 5910 were independent (average redundancy 3.613, completeness $\left.=99.8 \%, \mathrm{R}_{\text {int }}=3.96 \%, \mathrm{R}_{\text {sig }}=4.02 \%\right)$ and $4605(77.92 \%)$ were greater than $2 \sigma\left(\mathrm{F}^{2}\right)$. The final cell constants of $\underline{\mathrm{a}}=8.4295(3) \AA \stackrel{\AA}{\mathrm{b}}=10.9126(4) \AA \stackrel{\AA}{\mathrm{c}}=22.0700(9) \AA, \beta$ $=90.7067(13)^{\circ}$, volume $=2030.02(13) \AA^{3}$, are based upon the refinement of the XYZcentroids of 9831 reflections above $20 \sigma(\mathrm{I})$ with $6.107^{\circ}<2 \theta<64.95^{\circ}$. Data were corrected for absorption effects using the multi-scan method (SADABS). The ratio of minimum to maximum apparent transmission was 0.609. The calculated minimum and maximum transmission coefficients (based on crystal size) are 0.262 and 0.519 .

The structure was solved by direct methods and difference Fourier analysis using the programs provided by SHELXL-2013. ${ }^{\text {S2 }}$ Idealized positions for the hydrogen atoms were included as fixed contributions using a riding model with isotropic temperature factors set at 1.2 (aromatic and methine hydrogens) or 1.5 (methyl hydrogens) times that of the adjacent carbon atom. The positions of the methyl hydrogen atoms were optimized by a rigid rotating group refinement with idealized angles. Full-matrix least-squares refinement, based upon the minimization of $\Sigma \mathrm{w}_{\mathrm{i}}\left|\mathrm{F}_{\mathrm{o}}{ }^{2}-\mathrm{F}_{\mathrm{c}}{ }^{2}\right|^{2}$, with weighting $\mathrm{w}_{\mathrm{i}}^{-1}=\left[\sigma^{2}\left(\mathrm{~F}_{\mathrm{o}}^{2}\right)\right.$ $\left.+(0.0359 \mathrm{P})^{2}+1.0854 \mathrm{P}\right]$, where $\mathrm{P}=\left(\operatorname{Max}\left(\mathrm{F}_{\mathrm{o}}{ }^{2}, 0\right)+2 \mathrm{~F}_{\mathrm{c}}{ }^{2}\right) / 3 .{ }^{\mathrm{S} 2}$ The final anisotropic fullmatrix least-squares refinement on $\mathrm{F}^{2}$ with 255 variables converged at $\mathrm{R} 1=3.48 \%$ for 4605 observed data with $\mathrm{I}>2 \sigma(\mathrm{I})$ and $\mathrm{wR} 2=8.11 \%$ for all data. The goodness-of-fit was $1.036 .{ }^{\mathrm{S} 3}$

A correction for secondary extinction was not applied. The largest peak in the final difference electron density synthesis was $0.994 \mathrm{e}^{-} / \AA^{3}$ and the largest hole was $-0.825 \mathrm{e}^{-} / \AA^{3}$ with an RMS deviation of $0.092 \mathrm{e}^{-} / \AA^{3}$. The linear absorption coefficient, atomic scattering factors, and anomalous dispersion corrections were calculated from values found in the International Tables of X-ray Crystallography. ${ }^{\mathrm{S} 4}$ 
Table 2. Crystal data for $3\left(\mathrm{C}_{22} \mathrm{H}_{18} \mathrm{Br}_{2} \mathrm{O}_{4}\right)$.

$\begin{array}{ll}\text { Identification code } & \mathrm{kw} 26 \mathrm{cms} \\ \text { Chemical formula } & \mathrm{C}_{22} \mathrm{H}_{18} \mathrm{Br}_{2} \mathrm{O}_{4} \\ \text { Formula weight } & 506.18 \mathrm{~g} / \mathrm{mol} \\ \text { Temperature } & 105(2) \mathrm{K} \\ \text { Wavelength } & 0.71073 \AA \\ \text { Crystal size } & 0.188 \times 0.296 \times 0.456 \mathrm{~mm} \\ \text { Crystal system } & \text { monoclinic } \\ \text { Space group } & \mathrm{P} 2{ }_{1} / \mathrm{n}\left(\text { non-standard setting of } \mathrm{P} 2{ }_{1} / \mathrm{c}, \text { No. 14) }\right. \\ \text { Unit cell dimensions } & \mathrm{a}=8.4295(3) \AA \\ & \mathrm{b}=10.9126(4) \AA \quad \quad \beta=90^{\circ} \\ & \mathrm{c}=22.0700(9) \AA \quad \gamma=90^{\circ} \\ \text { Volume } & 2030.02(13) \AA^{3} \\ \text { Z } & 4 \\ \text { Density (calculated) } & 1.656 \mathrm{~g} / \mathrm{cm}^{3} \\ \text { Absorption coefficient } & 4.019 \mathrm{~mm}^{-1} \\ \text { F(000) } & 1008\end{array}$

Table 3. Data collection and structure refinement for $3\left(\mathrm{C}_{22} \mathrm{H}_{18} \mathrm{Br}_{2} \mathrm{O}_{4}\right)$.

\begin{tabular}{|c|c|}
\hline $\begin{array}{l}\text { Theta range used for } \\
\text { refinement }\end{array}$ & 3.05 to $30.00^{\circ}$ \\
\hline Index ranges & $-11 \leq \mathrm{h} \leq 9,-14 \leq \mathrm{k} \leq 15,-31 \leq 1 \leq 31$ \\
\hline Reflections collected & 21353 \\
\hline Independent reflections & $5910[\mathrm{R}(\mathrm{int})=0.0396]$ \\
\hline $\begin{array}{l}\text { Coverage of independent } \\
\text { reflections }\end{array}$ & $99.8 \%$ \\
\hline Absorption correction & multi-scan \\
\hline Max. and min. trans. & 0.519 and 0.262 \\
\hline Refinement method & Full-matrix least-squares on $\mathrm{F}^{2}$ \\
\hline Refinement program & SHELXL-2013 (Sheldrick, 2013) \\
\hline $\begin{array}{l}\text { Data / restraints / } \\
\text { parameters }\end{array}$ & $5910 / 0 / 255$ \\
\hline Goodness-of-fit on $\mathrm{F}^{2}$ & 1.036 \\
\hline
\end{tabular}


Final R indices

Largest diff. peak and hole
4605 data; $\mathrm{I}>2 \sigma(\mathrm{I}) \quad \mathrm{R} 1=0.0348, \mathrm{wR} 2=0.0727$

all data

0.994 and $-0.825 \mathrm{e}^{-} / \AA^{3}$

Table 4. Atomic coordinates and equivalent isotropic atomic displacement parameters $\left(\AA^{2}\right)$ for $3\left(\mathrm{C}_{22} \mathrm{H}_{18} \mathrm{Br}_{2} \mathrm{O}_{4}\right)$. U(eq) is defined as one third of the trace of the orthogonalized $\mathrm{U}_{\mathrm{ij}}$ tensor.

$\mathrm{x} / \mathrm{a}$

Br1 0.77944(2)

$\mathrm{Br} 2 \quad 0.28802(2)$

O1 $0.26530(13)$

$\mathrm{O} 2 \quad 0.02212(12)$

O3 0.39941(13)

O4 0.30747(12)

C1 0.04149(16)

$\mathrm{C} 2 \quad 0.11983(16)$

C3 0.04390(16)

$\mathrm{C} 4 \quad 0.86908(16)$

C5 0.79198(17)

C6 $0.86865(16)$

C7 $0.12459(17)$

C8 0.09028(19)

C9 0.29222(17)

C10 0.46887(19)

C11 0.84942(16)

C12 0.92926(17)

C13 0.91082(17)

C14 0.80942(17)

C15 0.72729(18)

C16 0.74879(18)

C17 0.11809(16)

C18 0.11192(17)

C19 0.16259(18) $\mathrm{y} / \mathrm{b}$

$0.14447(2)$

$0.49892(2)$

$0.61208(11)$

$0.67070(11)$

$0.50818(12)$

$0.36437(9)$

$0.56568(13)$

$0.48910(12)$

$0.41803(13)$

$0.44403(13)$

$0.52346(12)$

$0.59930(13)$

$0.61795(13)$

$0.72456(14)$

$0.45912(14)$

$0.32918(17)$

$0.73517(13)$

$0.78407(14)$

$0.90633(14)$

$0.97833(12)$

$0.93217(14)$

$0.80998(14)$

$0.43616(13)$

$0.34130(13)$

$0.35806(14)$ z/c

$0.17926(2)$

$0.45078(2)$

$0.03899(5)$

$0.00905(5)$

$0.15573(6)$

$0.09136(5)$

$0.10108(6)$

$0.13777(6)$

$0.18849(6)$

$0.19202(6)$

$0.15767(7)$

$0.10923(7)$

$0.04738(7)$

$0.95550(7)$

$0.12895(7)$

$0.07780(9)$

$0.12457(6)$

$0.17438(7)$

0.19074(7)

$0.15650(7)$

$0.10708(7)$

$0.09098(7)$

$0.25119(7)$

$0.29308(7)$

$0.35268(7)$
$\mathrm{U}(\mathrm{eq})$

0.02384(4)

$0.03687(5)$

$0.0276(3)$

$0.0216(3)$

$0.0312(3)$

$0.0215(2)$

0.0144(3)

$0.0142(3)$

$0.0145(3)$

$0.0153(3)$

$0.0160(3)$

$0.0155(3)$

$0.0163(3)$

$0.0229(4)$

$0.0180(3)$

$0.0349(5)$

$0.0152(3)$

$0.0181(3)$

$0.0179(3)$

$0.0172(3)$

$0.0223(3)$

$0.0211(3)$

$0.0149(3)$

$0.0180(3)$

0.0214(3) 


$\begin{array}{ccccc} & \mathrm{x} / \mathrm{a} & \mathrm{y} / \mathrm{b} & \mathrm{z} / \mathrm{c} & \mathrm{U}(\mathrm{eq}) \\ \mathrm{C} 20 & 0.22006(18) & 0.47167(14) & 0.36971(7) & 0.0212(3) \\ \mathrm{C} 21 & 0.22983(18) & 0.56726(14) & 0.32880(7) & 0.0197(3) \\ \mathrm{C} 22 & 0.17929(17) & 0.54925(13) & 0.26953(7) & 0.0170(3)\end{array}$

Table 5. Interatomic distances $(\AA)$ for $\mathbf{3}\left(\mathrm{C}_{22} \mathrm{H}_{18} \mathrm{Br}_{2} \mathrm{O}_{4}\right)$.

$\begin{array}{llll}\text { Br1-C14 } & 1.8990(14) & \text { Br2-C20 } & 1.8953(16) \\ \text { O1-C7 } & 1.2045(18) & \text { O2-C7 } & 1.3322(18) \\ \text { O2-C8 } & 1.4453(18) & \text { O3-C9 } & 1.1996(19) \\ \text { O4-C9 } & 1.3330(18) & \text { O4-C10 } & 1.4484(19) \\ \text { C1-C2 } & 1.333(2) & \text { C1-C7 } & 1.497(2) \\ \text { C1-C6 } & 1.5153(19) & \text { C2-C9 } & 1.5045(19) \\ \text { C2-C3 } & 1.5105(19) & \text { C3-C4 } & 1.5037(19) \\ \text { C3-C17 } & 1.524(2) & \text { C4-C5 } & 1.318(2) \\ \text { C5-C6 } & 1.504(2) & \text { C6-C11 } & 1.530(2) \\ \text { C11-C16 } & 1.386(2) & \text { C11-C12 } & 1.388(2) \\ \text { C12-C13 } & 1.391(2) & \text { C13-C14 } & 1.380(2) \\ \text { C14-C15 } & 1.380(2) & \text { C15-C16 } & 1.392(2) \\ \text { C17-C18 } & 1.389(2) & \text { C17-C22 } & 1.396(2) \\ \text { C18-C19 } & 1.390(2) & \text { C19-C20 } & 1.381(2) \\ \text { C20-C21 } & 1.383(2) & \text { C21-C22 } & 1.385(2)\end{array}$


Table 6. Bond angles $\left({ }^{\circ}\right)$ for $\mathbf{3}\left(\mathrm{C}_{22} \mathrm{H}_{18} \mathrm{Br}_{2} \mathrm{O}_{4}\right)$.

$\begin{array}{llll}\text { C7-O2-C8 } & 115.70(12) & \text { C9-O4-C10 } & 115.61(12) \\ \text { C2-C1-C7 } & 119.12(12) & \text { C2-C1-C6 } & 123.33(13) \\ \text { C7-C1-C6 } & 117.50(12) & \text { C1-C2-C9 } & 121.99(13) \\ \text { C1-C2-C3 } & 124.11(12) & \text { C9-C2-C3 } & 113.77(12) \\ \text { C4-C3-C2 } & 111.47(12) & \text { C4-C3-C17 } & 108.66(11) \\ \text { C2-C3-C17 } & 115.67(11) & \text { C5-C4-C3 } & 124.83(13) \\ \text { C4-C5-C6 } & 123.89(13) & \text { C5-C6-C1 } & 111.93(12) \\ \text { C5-C6-C11 } & 109.17(11) & \text { C1-C6-C11 } & 111.44(11) \\ \text { O1-C7-O2 } & 123.88(14) & \text { O1-C7-C1 } & 124.94(14) \\ \text { O2-C7-C1 } & 111.18(12) & \text { O3-C9-O4 } & 125.10(13) \\ \text { O3-C9-C2 } & 124.20(14) & \text { O4-C9-C2 } & 110.48(12) \\ \text { C16-C11-C12 } & 119.00(13) & \text { C16-C11-C6 } & 121.23(13) \\ \text { C12-C11-C6 } & 119.73(13) & \text { C11-C12-C13 } & 121.29(13) \\ \text { C14-C13-C12 } & 118.32(14) & \text { C13-C14-C15 } & 121.77(13) \\ \text { C13-C14-Br1 } & 118.84(11) & \text { C15-C14-Br1 } & 119.37(11) \\ \text { C14-C15-C16 } & 119.06(14) & \text { C11-C16-C15 } & 120.56(14) \\ \text { C18-C17-C22 } & 118.85(13) & \text { C18-C17-C3 } & 119.28(12) \\ \text { C22-C17-C3 } & 121.61(13) & \text { C17-C18-C19 } & 121.21(13) \\ \text { C20-C19-C18 } & 118.56(14) & \text { C19-C20-C21 } & 121.52(15) \\ \text { C19-C20-Br2 } & 119.90(12) & \text { C21-C20-Br2 } & 118.57(12) \\ \text { C20-C21-C22 } & 119.32(14) & \text { C21-C22-C17 } & 120.52(14)\end{array}$


Table 7. Anisotropic atomic displacement parameters $\left(\AA^{2}\right)$ for $\mathbf{3}\left(\mathrm{C}_{22} \mathrm{H}_{18} \mathrm{Br}_{2} \mathrm{O}_{4}\right)$. The anisotropic atomic displacement factor exponent takes the form: $-2 \pi^{2}\left[\mathrm{~h}^{2} \mathrm{a}^{* 2}\right.$ $\left.\mathrm{U}_{11}+\ldots+2 \mathrm{~h} \mathrm{k} \mathrm{a}^{*} \mathrm{~b}^{*} \mathrm{U}_{12}\right]$.
$\mathrm{U}_{11}$
$\mathrm{U}_{22}$
$\mathrm{U}_{23}$
$\mathrm{U}_{13}$
$\mathrm{U}_{12}$

$\begin{array}{lllllll}\text { Br1 } & 0.03172(8) & 0.01365(6) & 0.02633(7) & 0.00238(6) & 0.00759(6) & 0.00086(6)\end{array}$

$\begin{array}{llllllll}\mathrm{Br} 2 & 0.05461(11) & 0.03754(9) & 0.01818(7) & 0.00456(7) & -0.01055(7) & -0.00565(8)\end{array}$

$\begin{array}{lllllll}\mathrm{O} 1 & 0.0165(5) & 0.0358(6) & 0.0309(6) & 0.0092(5) & 0.0103(5) & 0.0029(4)\end{array}$

$\begin{array}{llllllll}\mathrm{O} 2 & 0.0190(5) & 0.0326(6) & 0.0133(5) & 0.0023(4) & 0.0027(4) & 0.0018(4)\end{array}$

$\begin{array}{lllllll}\mathrm{O} 3 & 0.0108(5) & 0.0518(7) & 0.0309(6) & -0.0142(6) & 0.0009(4) & -0.0037(5)\end{array}$

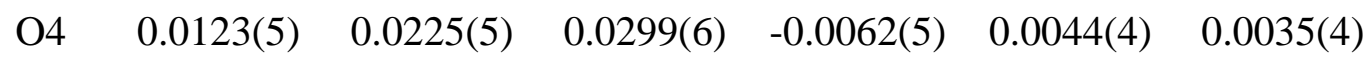

$\begin{array}{lllllll}\mathrm{C} 1 & 0.0109(6) & 0.0164(6) & 0.0159(6) & -0.0065(5) & 0.0033(5) & -0.0023(5)\end{array}$

$\begin{array}{lllllll}\mathrm{C} 2 & 0.0095(6) & 0.0177(6) & 0.0155(6) & -0.0054(5) & 0.0038(5) & -0.0023(5)\end{array}$

$\begin{array}{lllllll}\mathrm{C} 3 & 0.0110(6) & 0.0159(6) & 0.0166(6) & -0.0030(5) & 0.0033(5) & -0.0019(5)\end{array}$

$\begin{array}{lllllll}\mathrm{C} 4 & 0.0111(6) & 0.0168(6) & 0.0182(6) & -0.0076(5) & 0.0054(5) & -0.0047(5)\end{array}$

$\begin{array}{lllllll}\text { C5 } & 0.0107(6) & 0.0162(6) & 0.0211(7) & -0.0067(6) & 0.0048(5) & -0.0025(5)\end{array}$

$\begin{array}{lllllll}\text { C6 } & 0.0102(6) & 0.0195(6) & 0.0170(6) & -0.0042(6) & 0.0010(5) & 0.0000(5)\end{array}$

$\begin{array}{lllllll}\text { C7 } & 0.0177(7) & 0.0149(6) & 0.0165(6) & -0.0046(5) & 0.0034(5) & -0.0011(5)\end{array}$

$\begin{array}{lllllll}\text { C8 } & 0.0300(8) & 0.0248(7) & 0.0140(7) & 0.0002(6) & 0.0045(6) & -0.0009(6)\end{array}$

$\begin{array}{lllllll}\text { C9 } & 0.0119(6) & 0.0245(7) & 0.0175(6) & 0.0019(6) & 0.0039(5) & 0.0011(5)\end{array}$

$\begin{array}{llllllll}\mathrm{C} 10 & 0.0156(7) & 0.0427(9) & 0.0465(10) & -0.0101(9) & 0.0073(7) & 0.0114(7)\end{array}$

$\begin{array}{lllllll}\mathrm{C} 11 & 0.0120(6) & 0.0173(6) & 0.0165(6) & -0.0014(5) & 0.0054(5) & -0.0006(5)\end{array}$

$\begin{array}{llllllll}\mathrm{C} 12 & 0.0136(6) & 0.0206(6) & 0.0202(7) & -0.0013(6) & -0.0003(5) & 0.0027(5)\end{array}$

$\begin{array}{lllllll}\mathrm{C} 13 & 0.0156(6) & 0.0207(6) & 0.0175(6) & -0.0023(6) & 0.0029(5) & -0.0018(5)\end{array}$

$\begin{array}{lllllll}\mathrm{C} 14 & 0.0184(6) & 0.0141(6) & 0.0193(6) & 0.0014(6) & 0.0091(5) & -0.0005(5)\end{array}$

$\begin{array}{lllllll}\mathrm{C} 15 & 0.0237(7) & 0.0226(7) & 0.0206(7) & 0.0050(6) & 0.0001(6) & 0.0047(6)\end{array}$

$\begin{array}{lllllll}\mathrm{C} 16 & 0.0212(7) & 0.0255(7) & 0.0165(7) & -0.0011(6) & -0.0012(6) & 0.0020(6)\end{array}$

$\begin{array}{lllllll}\mathrm{C} 17 & 0.0098(6) & 0.0166(6) & 0.0183(6) & -0.0013(5) & 0.0039(5) & 0.0006(5)\end{array}$

$\begin{array}{llllllll}\mathrm{C} 18 & 0.0138(6) & 0.0164(6) & 0.0239(7) & 0.0001(6) & 0.0034(5) & -0.0009(5)\end{array}$

$\begin{array}{lllllll}\mathrm{C} 19 & 0.0202(7) & 0.0221(7) & 0.0219(7) & 0.0064(6) & 0.0023(6) & 0.0016(6)\end{array}$

$\begin{array}{lllllll}\mathrm{C} 20 & 0.0206(7) & 0.0265(7) & 0.0166(7) & 0.0026(6) & 0.0000(6) & 0.0026(6)\end{array}$

$\begin{array}{lllllll}\mathrm{C} 21 & 0.0207(7) & 0.0184(6) & 0.0200(7) & -0.0022(6) & -0.0013(6) & 0.0004(6)\end{array}$

$\begin{array}{llllllll}\mathrm{C} 22 & 0.0163(7) & 0.0161(6) & 0.0187(7) & 0.0021(6) & 0.0001(5) & -0.0005(5)\end{array}$ 
Table 8. Hydrogen atom coordinates and isotropic atomic displacement parameters $\left(\AA^{2}\right)$ for $3\left(\mathrm{C}_{22} \mathrm{H}_{18} \mathrm{Br}_{2} \mathrm{O}_{4}\right)$.

$\begin{array}{ccccc} & \mathrm{x} / \mathrm{a} & \mathrm{y} / \mathrm{b} & \mathrm{z} / \mathrm{c} & \mathrm{U}(\mathrm{eq}) \\ \text { H3 } & 0.0551 & 0.3292 & 0.1784 & 0.017 \\ \text { H4 } & -0.1902 & 0.3999 & 0.2212 & 0.018 \\ \text { H5 } & -0.3186 & 0.5334 & 0.1639 & 0.019 \\ \text { H6 } & -0.1887 & 0.5832 & 0.0701 & 0.019 \\ \text { H8A } & 0.1675 & 0.7875 & -0.0325 & 0.034 \\ \text { H8B } & 0.0060 & 0.7619 & -0.0693 & 0.034 \\ \text { H8C } & 0.1435 & 0.6608 & -0.0680 & 0.034 \\ \text { H10A } & 0.5196 & 0.3943 & 0.0544 & 0.052 \\ \text { H10B } & 0.4680 & 0.2532 & 0.0541 & 0.052 \\ \text { H10C } & 0.5283 & 0.3162 & 0.1157 & 0.052 \\ \text { H12 } & -0.0022 & 0.7330 & 0.1977 & 0.022 \\ \text { H13 } & -0.0334 & 0.9394 & 0.2246 & 0.022 \\ \text { H15 } & -0.3429 & 0.9830 & 0.0844 & 0.027 \\ \text { H16 } & -0.3060 & 0.7776 & 0.0567 & 0.025 \\ \text { H18 } & 0.0724 & 0.2636 & 0.2808 & 0.022 \\ \text { H19 } & 0.1578 & 0.2928 & 0.3811 & 0.026 \\ \text { H21 } & 0.2708 & 0.6445 & 0.3412 & 0.024 \\ \text { H22 } & 0.1863 & 0.6144 & 0.2411 & 0.02\end{array}$




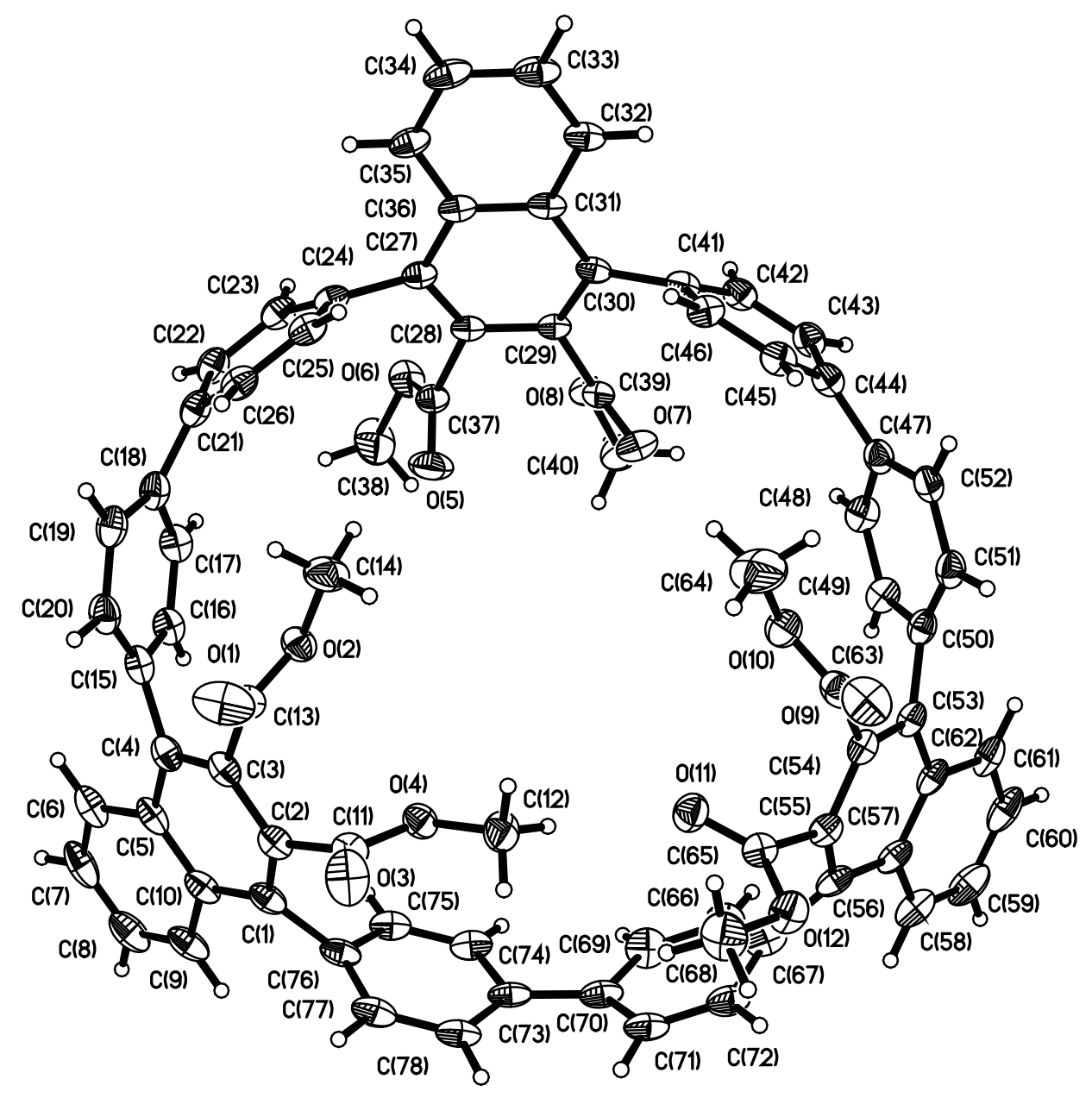

Figure S2. ORTEP Drawing of the Crystal Structure of 14.

Perspective view of the molecular structure of $\mathbf{1 4}\left(\mathrm{C}_{78} \mathrm{H}_{54} \mathrm{O}_{12}\right)$ with the atom labeling scheme for the independent non-hydrogen atoms. The thermal ellipsoids are scaled to enclose $30 \%$ probability. 


\section{Description of the X-ray Structural Analysis of $14\left(\mathrm{C}_{78} \mathrm{H}_{54} \mathrm{O}_{12} \cdot \mathrm{C}_{6} \mathrm{H}_{12} \cdot \mathrm{CH}_{2} \mathrm{Cl}_{2}\right)$}

A colorless crystal of $\mathbf{1 4}\left(\mathrm{C}_{78} \mathrm{H}_{54} \mathrm{O}_{12} \cdot 2 \mathrm{C}_{6} \mathrm{H}_{12} \cdot \mathrm{CH}_{2} \mathrm{Cl}_{2}\right)$ was covered in a polybutene oil (Sigma-Aldrich) and placed on the end of a MiTeGen loop. The sample was cooled to 173.15 K with an Oxford Cryostream 700 system and optically aligned on a Bruker AXS D8 Venture fixed-chi X-ray diffractometer equipped with a Triumph monochromator, a Mo K $\alpha$ radiation source $(\lambda=0.71073 \AA)$, and a PHOTON 100 CMOS detector. Two sets of 12 frames each were collected using the omega scan method with a 10 s exposure time. Integration of these frames followed by reflection indexing and least-squares refinement produced a crystal orientation matrix for the triclinic crystal lattice.

Data collection consisted of the measurement of a total of 324 frames in three runs using omega scans with the detector held at $5.00 \mathrm{~cm}$ from the crystal. Because the crystal did not diffract appreciably beyond $50^{\circ}$, it was necessary to use a longer exposure time. Also because the first crystals showed visual signs of internal fractures at $100 \mathrm{~K}$, the temperature of the cold stream was set at 173.15 during data collection. Frame scan parameters are summarized in Table 1 below:

Table 1. Data collection details for $\mathbf{1 4}\left(\mathrm{C}_{78} \mathrm{H}_{54} \mathrm{O}_{12} \cdot 2 \mathrm{C}_{6} \mathrm{H}_{12} \cdot \mathrm{CH}_{2} \mathrm{Cl}_{2}\right)$.

$\begin{array}{cccccccc}\text { Run } & 2 \theta & \omega & \varphi & \chi & \text { Scan Width }\left(^{\circ}\right) & \text { Frames } & \begin{array}{c}\text { Exposure Time } \\ (\mathrm{sec})\end{array} \\ 1 & 11.01 & -170.79 & 144.00 & 54.74 & 1.70 & 108 & 90.00 \\ & 11.01 & -170.79 & 0.00 & 54.74 & 1.70 & 108 & 90.00 \\ & 11.01 & -170.79 & -144.00 & 54.74 & 1.70 & 108 & 90.00\end{array}$


The APEX2 software program (version 2014.1-7) ${ }^{\mathrm{S} 1}$ was used for diffractometer control, preliminary frame scans, indexing, orientation matrix calculations, least-squares refinement of cell parameters, and the data collection. The frames were integrated with the Bruker SAINT software package using a narrow-frame algorithm. The integration of the data using a triclinic unit cell yielded a total of 51851 reflections to a maximum $\theta$ angle of $26.50^{\circ}(0.80 \AA \AA$ resolution), of which 15272 were independent (average redundancy 3.395, completeness $\left.=96.5 \%, \mathrm{R}_{\text {int }}=3.67 \%, \mathrm{R}_{\text {sig }}=4.54 \%\right)$ and $9134(59.81 \%)$ were greater than $2 \sigma\left(\mathrm{F}^{2}\right)$. The final cell constants of $\underline{a}=14.0233(8) \AA, \underline{b}=16.2794(10) \AA, \underline{c}=19.2082(11)$ $\AA, \alpha=66.9176(15)^{\circ}, \beta=72.0505(16)^{\circ}, \gamma=77.5633(16)^{\circ}$, volume $=3815.2(4) \AA^{3}$, are based upon the refinement of the XYZ-centroids of 9945 reflections above $20 \sigma(\mathrm{I})$ with $5.861^{\circ}<$ $2 \theta<54.91^{\circ}$. Data were corrected for absorption effects using the multi-scan method (SADABS). The ratio of minimum to maximum apparent transmission was 0.909. The calculated minimum and maximum transmission coefficients (based on crystal size) are 0.938 and 0.967 .

The structure was solved by direct methods and difference Fourier analysis using the programs provided by SHELXL-2013. ${ }^{\text {S2 }}$ The crystallographic asymmetric contains one independent molecule of $\mathrm{C}_{78} \mathrm{H}_{54} \mathrm{O}_{12}$, two 50:50 disordered molecules of $\mathrm{C}_{6} \mathrm{H}_{12}$ and a ca. 75:25 molecule of $\mathrm{CH}_{2} \mathrm{Cl}_{2}$. Idealized positions for the hydrogen atoms were included as fixed contributions using a riding model with isotropic temperature factors set at 1.2 times (aromatic and methylene hydrogens) or 1.5 (methyl hydrogens) times that of the adjacent carbon atom. The positions of the methyl hydrogen atoms were optimized by a rigid rotating group refinement with idealized angles. The positions of the carbon atoms of the two disordered sites for each molecule of $\mathrm{C}_{6} \mathrm{H}_{12}$ were refined with all of the C-C 
bonds restrained to $1.54 \pm 0.01 \AA$; the positions of the $\mathrm{C}$ and two $\mathrm{Cl}$ atoms of the two disordered sites of the $\mathrm{CH}_{2} \mathrm{Cl}_{2}$ were refined with all of the $\mathrm{C}-\mathrm{Cl}$ bonds restraints to $1.74 \pm$ $0.01 \AA$. Unfortunately, the poor resolution of the atom coordinates and large thermal displacements of non-hydrogen atoms within the disordered $\mathrm{C}_{6} \mathrm{H}_{12}$ and $\mathrm{CH}_{2} \mathrm{Cl}_{2}$ molecules limits the precision observed for the structural refinement of these solvents molecules in the lattice. However, the refinement does establish their relative locations and identity of these solvent molecules in the crystal lattice. The two $\mathrm{C}_{6} \mathrm{H}_{12}$ molecules lie between neighboring $\mathrm{C}_{78} \mathrm{H}_{54} \mathrm{O}_{12}$ molecules, whereas the $\mathrm{CH}_{2} \mathrm{Cl}_{2}$ lies within the internal cavity of $\mathrm{C}_{78} \mathrm{H}_{54} \mathrm{O}_{12}$.

Full-matrix least-squares refinement, based upon the minimization of $\Sigma \mathrm{w}_{\mathrm{i}} \mid \mathrm{F}_{\mathrm{o}}{ }^{2}-$ $\left.\mathrm{F}_{\mathrm{c}}^{2}\right|^{2}$, with weighting $\mathrm{w}_{\mathrm{i}}^{-1}=\left[\sigma^{2}\left(\mathrm{~F}_{\mathrm{o}}^{2}\right)+(0.0374 \mathrm{P})^{2}+6.6902 \mathrm{P}\right]$, where $\mathrm{P}=\left(\mathrm{Max}\left(\mathrm{F}_{\mathrm{o}}^{2}, 0\right)+\right.$ $\left.2 \mathrm{~F}_{\mathrm{c}}{ }^{2}\right) / 3 .^{\mathrm{S} 2}$ The final anisotropic full-matrix least-squares refinement on $\mathrm{F}^{2}$ with 1088 variables converged at $\mathrm{R} 1=7.92 \%$ for 18241 observed data with $\mathrm{I}>2 \sigma(\mathrm{I})$ and $\mathrm{wR} 2=18.80 \%$ for all data. The goodness-of-fit was $1.048 .^{\mathrm{S} 3}$

A correction for secondary extinction was not applied. The largest peak in the final difference electron density synthesis was $0.737 \mathrm{e}^{-} / \AA^{3}$ and the largest hole was $-0.353 \mathrm{e}^{-} / \AA^{3}$ with an RMS deviation of $0.054 \mathrm{e}^{-} / \AA^{3}$. The linear absorption coefficient, atomic scattering factors, and anomalous dispersion corrections were calculated from values found in the International Tables of X-ray Crystallography. ${ }^{\mathrm{S} 4}$ 
Table 2. Crystal data for $\mathbf{1 4}\left(\mathrm{C}_{78} \mathrm{H}_{54} \mathrm{O}_{12} \cdot 2 \mathrm{C}_{6} \mathrm{H}_{12} \cdot \mathrm{CH}_{2} \mathrm{Cl}_{2}\right)$.

$\begin{array}{lll}\text { Identification code } & \mathrm{kw} 29 \mathrm{cms} \\ \text { Chemical formula } & \mathrm{C}_{91} \mathrm{H}_{80} \mathrm{Cl}_{2} \mathrm{O}_{12} & \\ \text { Formula weight } & 1436.45 \mathrm{~g} / \mathrm{mol} & \\ \text { Temperature } & 173(2) \mathrm{K} \\ \text { Wavelength } & 0.71073 \AA \\ \text { Crystal size } & 0.228 \times 0.371 \times 0.437 \mathrm{~mm} \\ \text { Crystal system } & \text { triclinic } & \\ \text { Space group } & \mathrm{P}-1(\mathrm{No} .2) & \\ \text { Unit cell dimensions } & \mathrm{a}=14.0233(8) \AA & \alpha=66.9176(15)^{\circ} \\ & \mathrm{b}=16.2794(10) \AA & \beta=72.0505(16)^{\circ} \\ & \mathrm{c}=19.2082(11) \AA & \gamma=77.5633(16)^{\circ} \\ \text { Volume } & 3815.2(4) \AA^{3} & \\ \text { Z } & 2 \\ \text { Density (calculated) } & 1.250 \mathrm{~g} / \mathrm{cm}^{3} & \\ \text { Absorption coefficient } & 0.149 \mathrm{~mm}^{-1} & \\ \text { F(000) } & 1512 & \end{array}$

Table 3. Data collection and structure refinement for $\mathbf{1 4}\left(\mathrm{C}_{78} \mathrm{H}_{54} \mathrm{O}_{12} \cdot 2 \mathrm{C}_{6} \mathrm{H}_{12} \cdot \mathrm{CH}_{2} \mathrm{Cl}_{2}\right)$.

Theta range used for refinement

Index ranges

Reflections collected

Independent reflections

Coverage of independent reflections

Absorption correction

Max. and min. trans.

Refinement method

Refinement program

Data / restraints /

parameters

Goodness-of-fit on $\mathrm{F}^{2}$
2.98 to $26.50^{\circ}$

$-17 \leq \mathrm{h} \leq 17,-20 \leq \mathrm{k} \leq 19,-24 \leq 1 \leq 24$

51851

$15272[$ R(int $)=0.0367]$

$96.5 \%$

multi-scan

0.967 and 0.938

Full-matrix least-squares on $\mathrm{F}^{2}$

SHELXL-2013 (Sheldrick, 2013)

15272 / 29 / 1088

1.048 
Final R indices

Largest diff. peak and hole
9134 data; $\mathrm{I}>2 \sigma(\mathrm{I}) \quad \mathrm{R} 1=0.0792, \mathrm{wR} 2=0.1516$

all data

$\mathrm{R} 1=0.1362, w \mathrm{R} 2=0.1880$

Table 4. Atomic coordinates and equivalent isotropic atomic displacement parameters $\left(\AA^{2}\right)$ for $\mathbf{1 4}\left(\mathrm{C}_{78} \mathrm{H}_{54} \mathrm{O}_{12} \cdot 2 \mathrm{C}_{6} \mathrm{H}_{12} \cdot \mathrm{CH}_{2} \mathrm{Cl}_{2}\right)$. U(eq) is defined as one third of the trace of the orthogonalized $\mathrm{U}_{\mathrm{ij}}$ tensor.

0.737 and $-0.353 \mathrm{e}^{-} / \AA^{3}$

$\mathrm{x} / \mathrm{a}$

O1 $0.48466(10)$

O2 0.62193(9)

O3 0.57537(10)

O4 0.71856(10)

O5 $0.86664(12)$

O6 0.94313(9)

O7 0.92526(10)

O8 $0.01937(9)$

O9 $0.66634(10)$

O10 0.73804(11)

O11 0.71797(10)

O12 0.70266(10)

C1 0.75897(13)

C2 $0.68175(13)$

C3 0.64414(13)

C4 0.68167(13)

C5 0.75191(13)

C6 $0.78571(15)$

C7 $0.85422(16)$

C8 $0.89229(17)$

C9 $0.86177(14)$

C10 0.79052(14)

C11 0.65067(13)

C12 0.70360(16)

C13 0.57261(14)

\begin{abstract}
$\mathrm{y} / \mathrm{b}$
\end{abstract}
$0.70154(10)$

$0.62503(8)$

$0.88957(12)$

$0.82023(8)$

0.40501(9)

$0.26551(10)$

$0.46068(8)$

$0.33783(8)$

$0.90556(11)$

$0.77941(9)$

$0.92482(9)$

$0.06156(9)$

$0.81890(11)$

$0.79103(12)$

$0.70859(12)$

$0.65312(12)$

$0.68574(12)$

$0.63575(14)$

$0.66800(16)$

$0.74920(15)$

$0.79821(13)$

$0.76843(12)$

$0.84076(12)$

$0.86943(15)$

$0.67812(12)$ z/c

$0.22095(10)$

$0.17176(7)$

$0.10830(9)$

$0.05726(7)$

$0.07833(9)$

$0.11488(7)$

$0.87832(8)$

$0.93507(7)$

$0.65690(9)$

$0.73115(8)$

$0.80836(8)$

$0.71871(8)$

$0.20425(10)$

$0.19056(10)$

$0.23906(10)$

$0.30188(10)$

$0.32312(9)$

$0.39192(10)$

$0.41019(11)$

$0.36152(12)$

$0.29521(10)$

$0.27403(10)$

$0.11548(10)$

$0.97954(11)$

$0.21150(11)$
$\mathrm{U}(\mathrm{eq})$

0.0866(5)

0.0512(4)

$0.0773(6)$

$0.0515(4)$

$0.0770(5)$

$0.0600(4)$

$0.0595(4)$

$0.0483(4)$

$0.0756(5)$

$0.0631(4)$

$0.0569(4)$

0.0596(5)

$0.0458(5)$

$0.0432(5)$

$0.0421(5)$

$0.0444(5)$

$0.0474(5)$

$0.0601(6)$

$0.0704(7)$

$0.0715(6)$

$0.0592(5)$

$0.0481(5)$

$0.0463(5)$

$0.0637(6)$

$0.0479(5)$ 


\begin{tabular}{|c|c|c|c|c|}
\hline & $\mathrm{x} / \mathrm{a}$ & $y / b$ & $\mathrm{z} / \mathrm{c}$ & $\mathrm{U}(\mathrm{eq}$ \\
\hline C14 & $0.56032(17)$ & $0.59182(14)$ & $0.14226(12)$ & $0.0707(7)$ \\
\hline C15 & $0.66665(14)$ & $0.55590(13)$ & $0.33247(10)$ & $0.0481(6)$ \\
\hline $\mathrm{C} 16$ & $0.75125(14)$ & $0.49576(13)$ & $0.31943(10)$ & $0.0488(6)$ \\
\hline $\mathrm{C} 17$ & $0.74248(14)$ & $0.41494(13)$ & $0.31626(10)$ & $0.0508(6)$ \\
\hline C18 & $0.64888(15)$ & $0.38958(13)$ & $0.32644(11)$ & $0.0495(6)$ \\
\hline C19 & $0.56409(15)$ & $0.44350(14)$ & $0.35190(11)$ & $0.0561(7)$ \\
\hline $\mathrm{C} 20$ & $0.57298(15)$ & $0.52536(14)$ & $0.35461(11)$ & $0.0550(6)$ \\
\hline $\mathrm{C} 21$ & $0.64650(14)$ & $0.32481(13)$ & $0.29042(11)$ & $0.0486(6)$ \\
\hline $\mathrm{C} 22$ & $0.72159(14)$ & $0.25311(13)$ & $0.28717(11)$ & $0.0504(6)$ \\
\hline $\mathrm{C} 23$ & $0.74344(14)$ & $0.21912(12)$ & $0.22729(11)$ & $0.0489(6)$ \\
\hline $\mathrm{C} 24$ & $0.69247(13)$ & $0.25582(11)$ & $0.16849(11)$ & $0.0428(5)$ \\
\hline $\mathrm{C} 25$ & $0.60596(13)$ & $0.31582(12)$ & $0.17954(11)$ & $0.0464(5)$ \\
\hline $\mathrm{C} 26$ & $0.58361(14)$ & $0.34882(12)$ & $0.23959(11)$ & $0.0495(6)$ \\
\hline $\mathrm{C} 27$ & $0.73580(12)$ & $0.25382(10)$ & $0.08756(10)$ & $0.0395(5)$ \\
\hline $\mathrm{C} 28$ & $0.82186(12)$ & $0.29585(10)$ & $0.04412(10)$ & $0.0392(5)$ \\
\hline $\mathrm{C} 29$ & $0.84868(12)$ & $0.32494(10)$ & $0.96112(10)$ & $0.0379(5)$ \\
\hline C30 & $0.79110(12)$ & $0.30982(10)$ & $0.92173(10)$ & $0.0393(5)$ \\
\hline C31 & $0.70885(12)$ & $0.25711(10)$ & $0.96528(10)$ & $0.0405(5)$ \\
\hline C32 & $0.65286(13)$ & $0.23068(11)$ & $0.92859(11)$ & $0.0482(5)$ \\
\hline C33 & $0.57590(14)$ & $0.17854(12)$ & $0.97175(12)$ & $0.0594(6)$ \\
\hline C34 & $0.54989(14)$ & $0.15031(12)$ & $0.05312(12)$ & $0.0586(6)$ \\
\hline $\mathrm{C} 35$ & $0.60009(13)$ & $0.17581(11)$ & $0.09067(12)$ & $0.0485(6)$ \\
\hline C36 & $0.68131(12)$ & $0.22878(10)$ & $0.04857(10)$ & $0.0409(5)$ \\
\hline C37 & $0.87838(14)$ & $0.32887(12)$ & $0.08126(11)$ & $0.0489(5)$ \\
\hline C38 & $0.00188(17)$ & $0.29339(19)$ & $0.15083(13)$ & $0.0973(9)$ \\
\hline C39 & $0.93374(13)$ & $0.38339(11)$ & $0.91896(10)$ & $0.0409(5)$ \\
\hline $\mathrm{C} 40$ & $0.10704(15)$ & $0.38814(15)$ & $0.90167(14)$ & $0.0688(8)$ \\
\hline C41 & $0.80787(13)$ & $0.36175(11)$ & $0.83630(10)$ & $0.0433(5)$ \\
\hline $\mathrm{C} 42$ & $0.90093(14)$ & $0.35931(12)$ & $0.78344(10)$ & $0.0482(5)$ \\
\hline C43 & $0.92568(15)$ & $0.43093(12)$ & $0.71540(10)$ & $0.0498(6)$ \\
\hline C44 & $0.85718(14)$ & $0.50831(12)$ & $0.69719(10)$ & $0.0466(5)$ \\
\hline $\mathrm{C} 45$ & $0.75891(14)$ & $0.50283(12)$ & $0.74376(10)$ & $0.0486(5)$ \\
\hline C46 & $0.73392(13)$ & $0.43127(12)$ & $0.81202(10)$ & $0.0463(5)$ \\
\hline $\mathrm{C} 47$ & $0.89093(15)$ & $0.59776(12)$ & $0.64733(10)$ & $0.0462(5)$ \\
\hline C48 & $0.97364(14)$ & $0.61975(13)$ & $0.65959(11)$ & $0.0508(6)$ \\
\hline
\end{tabular}




\begin{tabular}{|c|c|c|c|c|}
\hline & $\mathrm{x} / \mathrm{a}$ & $\mathrm{y} / \mathrm{b}$ & $\mathrm{z} / \mathrm{c}$ & $\mathrm{U}(\mathrm{eq}$ \\
\hline C49 & $0.99064(14)$ & $0.70758(13)$ & $0.63767(11)$ & $0.0497(6)$ \\
\hline $\mathrm{C} 50$ & $0.92461(13)$ & $0.77801(12)$ & $0.60255(10)$ & $0.0431(5)$ \\
\hline C51 & $0.85053(15)$ & $0.75647(13)$ & $0.58057(10)$ & $0.0493(6)$ \\
\hline C52 & $0.83360(15)$ & $0.66806(13)$ & $0.60281(10)$ & $0.0488(5)$ \\
\hline C53 & $0.92306(13)$ & $0.86585(12)$ & $0.61018(10)$ & $0.0427(5)$ \\
\hline C54 & $0.83793(13)$ & $0.89766(12)$ & $0.65559(10)$ & $0.0418(5)$ \\
\hline C55 & $0.84233(13)$ & $0.96189(11)$ & $0.68686(10)$ & $0.0412(5)$ \\
\hline C56 & $0.93250(13)$ & $0.99152(11)$ & $0.67632(11)$ & $0.0455(5)$ \\
\hline C57 & $0.01795(14)$ & $0.97027(12)$ & $0.61967(11)$ & $0.0479(6)$ \\
\hline C58 & $0.10984(15)$ & $0.00840(14)$ & $0.59620(13)$ & $0.0611(7)$ \\
\hline C59 & $0.19095(16)$ & $0.98679(15)$ & $0.54285(14)$ & $0.0717(8)$ \\
\hline C60 & $0.18491(16)$ & $0.92628(15)$ & $0.50987(13)$ & $0.0674(8)$ \\
\hline C61 & $0.09875(14)$ & $0.88744(14)$ & $0.53058(11)$ & $0.0544(7)$ \\
\hline C62 & $0.01338(13)$ & $0.90770(12)$ & $0.58643(11)$ & $0.0452(6)$ \\
\hline C63 & $0.73865(15)$ & $0.86117(14)$ & $0.68075(11)$ & $0.0569(6)$ \\
\hline C64 & $0.6394(2)$ & $0.74696(19)$ & $0.75449(19)$ & $0.1118(11)$ \\
\hline C65 & $0.74890(13)$ & $0.97961(12)$ & $0.74568(10)$ & $0.0443(5)$ \\
\hline C66 & $0.61120(17)$ & $0.08224(16)$ & $0.77333(14)$ & $0.0750(8)$ \\
\hline C67 & $0.94597(14)$ & $0.01933(12)$ & $0.73786(12)$ & $0.0517(6)$ \\
\hline C68 & $0.01684(15)$ & $0.96564(14)$ & $0.77794(12)$ & $0.0618(7)$ \\
\hline C69 & $0.01103(15)$ & $0.96031(15)$ & $0.85221(13)$ & $0.0639(7)$ \\
\hline $\mathrm{C} 70$ & $0.93287(13)$ & $0.00777(11)$ & $0.89123(11)$ & $0.0505(5)$ \\
\hline C71 & $0.87419(15)$ & $0.07268(12)$ & $0.84533(12)$ & $0.0583(6)$ \\
\hline $\mathrm{C} 72$ & $0.87980(16)$ & $0.07882(12)$ & $0.77056(12)$ & $0.0587(6)$ \\
\hline C73 & $0.90483(12)$ & $0.97736(11)$ & $0.97757(11)$ & $0.0474(5)$ \\
\hline C74 & $0.95246(13)$ & $0.89775(12)$ & $0.02100(12)$ & $0.0513(6)$ \\
\hline C75 & $0.91084(13)$ & $0.85418(12)$ & $0.09849(11)$ & $0.0495(5)$ \\
\hline C76 & $0.81804(13)$ & $0.88706(11)$ & $0.13686(10)$ & $0.0472(5)$ \\
\hline $\mathrm{C} 77$ & $0.77697(15)$ & $0.97204(12)$ & $0.09696(11)$ & $0.0571(6)$ \\
\hline $\mathrm{C} 78$ & $0.82048(14)$ & $0.01639(12)$ & $0.01934(11)$ & $0.0549(5)$ \\
\hline C79 & $0.5906(5)$ & $0.9372(4)$ & $0.4134(2)$ & $0.220(3)$ \\
\hline $\mathrm{C} 80$ & $0.5609(3)$ & $0.9126(3)$ & $0.3549(2)$ & $0.105(2)$ \\
\hline C81 & $0.4706(4)$ & $0.8606(4)$ & $0.4103(3)$ & $0.141(3)$ \\
\hline $\mathrm{C} 82$ & $0.5038(6)$ & $0.7710(4)$ & $0.4702(3)$ & $0.250(5)$ \\
\hline $\mathrm{C} 83$ & $0.5354(3)$ & $0.8042(3)$ & $0.5235(2)$ & $0.136(2)$ \\
\hline
\end{tabular}




\begin{tabular}{lllll} 
& \multicolumn{1}{c}{$\mathrm{x} / \mathrm{a}$} & \multicolumn{1}{c}{$\mathrm{y} / \mathrm{b}$} & \multicolumn{1}{c}{$\mathrm{z} / \mathrm{c}$} & \multicolumn{1}{c}{$\mathrm{eq})$} \\
$\mathrm{C} 84$ & $0.6322(4)$ & $0.8475(4)$ & $0.4695(4)$ & $0.168(3)$ \\
C79' & $0.6130(4)$ & $0.9259(7)$ & $0.4121(3)$ & $0.344(7)$ \\
C80' & $0.5253(4)$ & $0.9248(3)$ & $0.3810(3)$ & $0.212(5)$ \\
C81' & $0.5192(6)$ & $0.8252(3)$ & $0.3976(3)$ & $0.156(3)$ \\
C82' & $0.4780(4)$ & $0.7923(6)$ & $0.4875(2)$ & $0.180(3)$ \\
C83' & $0.5665(4)$ & $0.7755(3)$ & $0.5249(4)$ & $0.241(7)$ \\
C84' & $0.5781(6)$ & $0.8759(3)$ & $0.5015(3)$ & $0.141(3)$ \\
C85 & $0.3158(3)$ & $0.6391(2)$ & $0.4534(2)$ & $0.0639(14)$ \\
C86 & $0.2518(3)$ & $0.7277(3)$ & $0.4186(4)$ & $0.128(3)$ \\
C87 & $0.1411(3)$ & $0.7141(3)$ & $0.4396(2)$ & $0.0885(18)$ \\
C88 & $0.1367(5)$ & $0.6488(3)$ & $0.4038(3)$ & $0.188(3)$ \\
C89 & $0.1923(3)$ & $0.5601(3)$ & $0.4451(3)$ & $0.1231(16)$ \\
C90 & $0.3036(3)$ & $0.5686(3)$ & $0.4235(3)$ & $0.156(3)$ \\
C85' & $0.1559(4)$ & $0.7178(3)$ & $0.3798(4)$ & $0.129(3)$ \\
C86' & $0.2641(3)$ & $0.6955(5)$ & $0.3356(2)$ & $0.116(3)$ \\
C87' & $0.3222(3)$ & $0.6921(3)$ & $0.3917(2)$ & $0.093(2)$ \\
C88' & $0.2938(4)$ & $0.6002(4)$ & $0.4548(4)$ & $0.389(7)$ \\
C89' & $0.1919(3)$ & $0.6360(4)$ & $0.5002(2)$ & $0.117(3)$ \\
C90' & $0.1282(3)$ & $0.6335(3)$ & $0.4499(2)$ & $0.0759(14)$ \\
C91 & $0.2683(3)$ & $0.4367(2)$ & $0.0511(3)$ & $0.120(2)$ \\
C11 & $0.27869(8)$ & $0.32482(7)$ & $0.06340(7)$ & $0.1026(4)$ \\
C12 & $0.38046(10)$ & $0.48035(7)$ & $0.03022(7)$ & $0.1144(4)$ \\
C91' & $0.2748(4)$ & $0.4415(3)$ & $0.0600(4)$ & $0.162(3)$ \\
C11' & $0.2816(4)$ & $0.3441(2)$ & $0.0437(3)$ & $0.232(2)$ \\
C12' & $0.40193(19)$ & $0.4481(3)$ & $0.0473(2)$ & $0.1827(16)$
\end{tabular}


Table 5. Interatomic distances $(\AA)$ for $\mathbf{1 4}\left(\mathrm{C}_{78} \mathrm{H}_{54} \mathrm{O}_{12}\right)$.

$\begin{array}{llll}\text { O1-C13 } & 1.189(2) & \text { O2-C13 } & 1.320(2) \\ \text { O2-C14 } & 1.450(3) & \text { O3-C11 } & 1.187(2) \\ \text { O4-C11 } & 1.330(2) & \text { O4-C12 } & 1.448(2) \\ \text { O5-C37 } & 1.196(2) & \text { O6-C37 } & 1.319(2) \\ \text { O6-C38 } & 1.456(3) & \text { O7-C39 } & 1.1955(19) \\ \text { O8-C39 } & 1.324(2) & \text { O8-C40 } & 1.459(2) \\ \text { O9-C63 } & 1.213(2) & \text { O10-C63 } & 1.303(2) \\ \text { O10-C64 } & 1.463(3) & \text { O11-C65 } & 1.198(2) \\ \text { O12-C65 } & 1.327(2) & \text { O12-C66 } & 1.456(3) \\ \text { C1-C2 } & 1.389(3) & \text { C1-C10 } & 1.419(2) \\ \text { C1-C76 } & 1.484(2) & \text { C2-C3 } & 1.403(2) \\ \text { C2-C11 } & 1.498(3) & \text { C3-C4 } & 1.372(2) \\ \text { C3-C13 } & 1.510(3) & \text { C4-C5 } & 1.436(3) \\ \text { C4-C15 } & 1.494(3) & \text { C5-C10 } & 1.414(2) \\ \text { C5-C6 } & 1.418(3) & \text { C6-C7 } & 1.375(3) \\ \text { C7-C8 } & 1.391(3) & \text { C8-C9 } & 1.354(3) \\ \text { C9-C10 } & 1.419(3) & \text { C15-C20 } & 1.387(3) \\ \text { C15-C16 } & 1.392(3) & \text { C16-C17 } & 1.374(3) \\ \text { C17-C18 } & 1.394(3) & \text { C18-C19 } & 1.397(3) \\ \text { C18-C21 } & 1.481(3) & \text { C19-C20 } & 1.387(3) \\ \text { C21-C22 } & 1.401(3) & \text { C21-C26 } & 1.402(3) \\ \text { C22-C23 } & 1.390(3) & \text { C23-C24 } & 1.388(3) \\ \text { C24-C25 } & 1.402(2) & \text { C24-C27 } & 1.495(3) \\ \text { C25-C26 } & 1.381(3) & \text { C27-C28 } & 1.381(2) \\ \text { C27-C36 } & 1.427(3) & \text { C28-C29 } & 1.422(2) \\ \text { C28-C37 } & 1.503(3) & \text { C29-C30 } & 1.376(3) \\ \text { C29-C39 } & 1.508(2) & \text { C30-C31 } & 1.427(2) \\ \text { C30-C41 } & 1.491(2) & \text { C31-C32 } & 1.422(3) \\ \text { C31-C36 } & 1.428(2) & \text { C32-C33 } & 1.365(2) \\ \text { C33-C34 } & 1.395(3) & \text { C34-C35 } & 1.364(3) \\ \text { C35-C36 } & 1.415(2) & \text { C41-C42 } & 1.389(2) \\ \text { C41-C46 } & 1.397(2) & \text { C42-C43 } & 1.374(2) \\ \text { C43-C44 } & 1.404(2) & \text { C44-C45 } & 1.392(2)\end{array}$




$\begin{array}{llll}\text { C44-C47 } & 1.479(2) & \text { C45-C46 } & 1.376(2) \\ \text { C47-C48 } & 1.394(3) & \text { C47-C52 } & 1.396(3) \\ \text { C48-C49 } & 1.375(3) & \text { C49-C50 } & 1.396(2) \\ \text { C50-C51 } & 1.387(3) & \text { C50-C53 } & 1.489(3) \\ \text { C51-C52 } & 1.383(3) & \text { C53-C54 } & 1.378(2) \\ \text { C53-C62 } & 1.431(3) & \text { C54-C55 } & 1.415(3) \\ \text { C54-C63 } & 1.497(3) & \text { C55-C56 } & 1.379(3) \\ \text { C55-C65 } & 1.502(2) & \text { C56-C57 } & 1.433(3) \\ \text { C56-C67 } & 1.494(3) & \text { C57-C62 } & 1.419(3) \\ \text { C57-C58 } & 1.420(3) & \text { C58-C59 } & 1.364(3) \\ \text { C59-C60 } & 1.392(4) & \text { C60-C61 } & 1.361(3) \\ \text { C61-C62 } & 1.417(3) & \text { C67-C68 } & 1.384(3) \\ \text { C67-C72 } & 1.387(3) & \text { C68-C69 } & 1.372(3) \\ \text { C69-C70 } & 1.394(3) & \text { C70-C71 } & 1.383(3) \\ \text { C70-C73 } & 1.479(3) & \text { C71-C72 } & 1.378(3) \\ \text { C73-C78 } & 1.392(3) & \text { C73-C74 } & 1.394(2) \\ \text { C74-C75 } & 1.369(2) & \text { C75-C76 } & 1.393(2) \\ \text { C76-C77 } & 1.389(2) & \text { C77-C78 } & 1.379(3)\end{array}$


Table 6. Bond angles $\left(^{\circ}\right)$ for $\mathbf{1 4}\left(\mathrm{C}_{78} \mathrm{H}_{54} \mathrm{O}_{12}\right)$.

\begin{tabular}{|c|c|c|c|}
\hline C13-O2-C14 & $115.33(15)$ & C11-O4-C12 & $116.53(14)$ \\
\hline C37-O6-C38 & $115.42(18)$ & C39-O8-C40 & $116.15(14)$ \\
\hline C63-O10-C64 & $111.48(18)$ & C65-O12-C66 & $114.36(14)$ \\
\hline C2-C1-C10 & $118.74(16)$ & C2-C1-C76 & $116.49(16)$ \\
\hline C10-C1-C76 & $123.29(17)$ & $\mathrm{C} 1-\mathrm{C} 2-\mathrm{C} 3$ & $121.02(16)$ \\
\hline C1-C2-C11 & $119.59(15)$ & C3-C2-C11 & $118.47(18)$ \\
\hline C4-C3-C2 & 121.27(19) & C4-C3-C13 & $121.63(16)$ \\
\hline C2-C3-C13 & $116.44(15)$ & C3-C4-C5 & $118.48(16)$ \\
\hline C3-C4-C15 & 117.59(19) & C5-C4-C15 & $122.33(16)$ \\
\hline C10-C5-C6 & $118.7(2)$ & C10-C5-C4 & $119.90(16)$ \\
\hline C6-C5-C4 & 121.41(17) & C7-C6-C5 & $120.16(19)$ \\
\hline C6-C7-C8 & 120.93(19) & C9-C8-C7 & $120.2(2)$ \\
\hline C8-C9-C10 & 121.11(19) & C5-C10-C1 & 119.64(19) \\
\hline C5-C10-C9 & $118.90(16)$ & C1-C10-C9 & $121.46(16)$ \\
\hline O3-C11-O4 & $124.10(18)$ & $\mathrm{O} 3-\mathrm{C} 11-\mathrm{C} 2$ & $125.74(17)$ \\
\hline $\mathrm{O} 4-\mathrm{C} 11-\mathrm{C} 2$ & $110.13(14)$ & $\mathrm{O} 1-\mathrm{C} 13-\mathrm{O} 2$ & $124.2(2)$ \\
\hline $\mathrm{O} 1-\mathrm{C} 13-\mathrm{C} 3$ & $125.1(2)$ & $\mathrm{O} 2-\mathrm{C} 13-\mathrm{C} 3$ & $110.65(15)$ \\
\hline C20-C15-C16 & $117.3(2)$ & C20-C15-C4 & $123.36(17)$ \\
\hline C16-C15-C4 & $117.28(16)$ & C17-C16-C15 & $121.07(19)$ \\
\hline C16-C17-C18 & $121.55(18)$ & C17-C18-C19 & $116.5(2)$ \\
\hline C17-C18-C21 & $117.52(17)$ & C19-C18-C21 & $123.8(2)$ \\
\hline C20-C19-C18 & 121.1(2) & C19-C20-C15 & $120.87(18)$ \\
\hline C22-C21-C26 & $116.5(2)$ & C22-C21-C18 & $122.9(2)$ \\
\hline C26-C21-C18 & 118.34(17) & $\mathrm{C} 23-\mathrm{C} 22-\mathrm{C} 21$ & $120.85(19)$ \\
\hline C24-C23-C22 & $121.08(17)$ & $\mathrm{C} 23-\mathrm{C} 24-\mathrm{C} 25$ & $117.47(19)$ \\
\hline $\mathrm{C} 23-\mathrm{C} 24-\mathrm{C} 27$ & $124.18(15)$ & $\mathrm{C} 25-\mathrm{C} 24-\mathrm{C} 27$ & $116.63(16)$ \\
\hline C26-C25-C24 & $120.35(19)$ & C25-C26-C21 & $121.64(17)$ \\
\hline C28-C27-C36 & 118.61(16) & C28-C27-C24 & $117.34(18)$ \\
\hline C36-C27-C24 & $122.58(15)$ & C27-C28-C29 & $121.10(18)$ \\
\hline C27-C28-C37 & $120.78(16)$ & C29-C28-C37 & $117.00(14)$ \\
\hline С30-C29-C28 & $120.94(15)$ & C30-C29-C39 & $121.82(15)$ \\
\hline С28-C29-C39 & $116.73(17)$ & C29-C30-C31 & $119.10(15)$ \\
\hline C29-C30-C41 & $117.44(15)$ & C31-C30-C41 & $122.71(17)$ \\
\hline
\end{tabular}




$\begin{array}{llll}\text { C32-C31-C30 } & 122.21(16) & \text { C32-C31-C36 } & 118.14(15) \\ \text { C30-C31-C36 } & 119.65(18) & \text { C33-C32-C31 } & 121.02(18) \\ \text { C32-C33-C34 } & 120.6(2) & \text { C35-C34-C33 } & 120.30(18) \\ \text { C34-C35-C36 } & 121.15(19) & \text { C35-C36-C27 } & 121.46(17) \\ \text { C35-C36-C31 } & 118.73(19) & \text { C27-C36-C31 } & 119.81(15) \\ \text { O5-C37-O6 } & 124.7(2) & \text { O5-C37-C28 } & 122.78(17) \\ \text { O6-C37-C28 } & 112.53(16) & \text { O7-C39-O8 } & 124.38(16) \\ \text { O7-C39-C29 } & 125.18(16) & \text { O8-C39-C29 } & 110.42(13) \\ \text { C42-C41-C46 } & 117.78(15) & \text { C42-C41-C30 } & 123.09(15) \\ \text { C46-C41-C30 } & 117.28(15) & \text { C43-C42-C41 } & 120.92(16) \\ \text { C42-C43-C44 } & 120.85(17) & \text { C45-C44-C43 } & 116.78(15) \\ \text { C45-C44-C47 } & 119.03(16) & \text { C43-C44-C47 } & 122.04(17) \\ \text { C46-C45-C44 } & 121.61(16) & \text { C45-C46-C41 } & 120.14(16) \\ \text { C48-C47-C52 } & 116.86(18) & \text { C48-C47-C44 } & 117.06(16) \\ \text { C52-C47-C44 } & 124.35(19) & \text { C49-C48-C47 } & 121.60(17) \\ \text { C48-C49-C50 } & 120.7(2) & \text { C51-C50-C49 } & 117.62(19) \\ \text { C51-C50-C53 } & 124.18(16) & \text { C49-C50-C53 } & 116.30(19) \\ \text { C52-C51-C50 } & 120.88(17) & \text { C51-C52-C47 } & 121.2(2) \\ \text { C54-C53-C62 } & 118.53(19) & \text { C54-C53-C50 } & 118.64(16) \\ \text { C62-C53-C50 } & 121.41(16) & \text { C53-C54-C55 } & 121.02(18) \\ \text { C53-C54-C63 } & 122.74(19) & \text { C55-C54-C63 } & 116.01(16) \\ \text { C56-C55-C54 } & 121.28(16) & \text { C56-C55-C65 } & 121.79(19) \\ \text { C54-C55-C65 } & 115.60(16) & \text { C55-C56-C57 } & 117.8(2) \\ \text { C55-C56-C67 } & 118.96(16) & \text { C57-C56-C67 } & 120.74(18) \\ \text { C62-C57-C58 } & 117.77(18) & \text { C62-C57-C56 } & 120.09(18) \\ \text { C58-C57-C56 } & 122.1(2) & \text { C59-C58-C57 } & 121.5(2) \\ \text { C58-C59-C60 } & 120.1(2) & \text { C61-C60-C59 } & 120.8(2) \\ \text { C60-C61-C62 } & 120.6(2) & \text { C61-C62-C57 } & 119.26(18) \\ \text { C61-C62-C53 } & 121.2(2) & \text { C57-C62-C53 } & 119.50(16) \\ \text { O9-C63-O10 } & 124.5(2) & \text { O9-C63-C54 } & 122.03(17) \\ \text { O10-C63-C54 } & 113.45(17) & \text { O11-C65-O12 } & 124.16(16) \\ \text { O11-C65-C55 } & 123.75(16) & \text { O12-C65-C55 } & 112.04(14) \\ \text { C68-C67-C72 } & 116.4(2) & \text { C68-C67-C56 } & 115.88(18) \\ \text { C72-C67-C56 } & 125.83(19) & \text { C69-C68-C67 } & 121.5(2) \\ \text { C71-C70-C73 } & 123.15(17) & \text { C69-C70-C73 } & 120.57(16)\end{array}$




$\begin{array}{llll}\text { C72-C71-C70 } & 122.37(19) & \text { C71-C72-C67 } & 120.71(18) \\ \text { C78-C73-C74 } & 116.35(17) & \text { C78-C73-C70 } & 122.59(15) \\ \text { C74-C73-C70 } & 120.17(16) & \text { C75-C74-C73 } & 121.58(17) \\ \text { C74-C75-C76 } & 121.14(16) & \text { C77-C76-C75 } & 117.25(15) \\ \text { C77-C76-C1 } & 124.17(16) & \text { C75-C76-C1 } & 115.72(15) \\ \text { C78-C77-C76 } & 120.67(17) & \text { C77-C78-C73 } & 121.73(16)\end{array}$


Table 7. Anisotropic atomic displacement parameters $\left(\AA^{2}\right)$ for $\mathbf{1 4}$ $\left(\mathrm{C}_{78} \mathrm{H}_{54} \mathrm{O}_{12} \cdot 2 \mathrm{C}_{6} \mathrm{H}_{12} \cdot \mathrm{CH}_{2} \mathrm{Cl}_{2}\right)$. The anisotropic atomic displacement factor

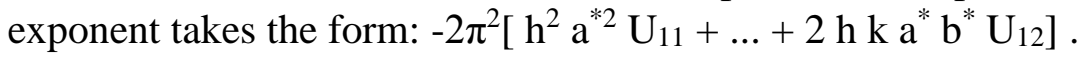

\begin{tabular}{|c|c|c|c|c|c|c|}
\hline & & & & & & \\
\hline $\mathrm{O} 1$ & $0.0420(7)$ & $0.0873(9)$ & $0.1565(11)$ & $-0.0676(7)$ & $-0.0432(7)$ & $0.0161(7)$ \\
\hline $\mathrm{O} 2$ & $0.0524(7)$ & $0.0596(6)$ & 0519(6) & $-0.0248(4)$ & $-0.0189(5)$ & $-0.0079(5)$ \\
\hline $\mathrm{O} 3$ & .0413 & $0.1095(11)$ & 0.073 & $-0.0283(8)$ & $-0.0271(6)$ & \\
\hline $\mathrm{O} 4$ & $0.0539(7)$ & $0.0575(6)$ & $0.0509(6)$ & $-0.0232(5)$ & $-0.0201(5)$ & $-0.0036(5)$ \\
\hline O5 & $0.0958(9)$ & $0.0617(7)$ & $0.0969(8)$ & $-0.0375(5)$ & $-0.0342(6)$ & $-0.0221(6)$ \\
\hline O6 & $0.0440(6)$ & $0.0848(8)$ & 0.0633 & $-0.0284(5)$ & $-0.0237(5)$ & -0.011 \\
\hline $\mathrm{O} 7$ & $0.0514(7)$ & $0.0431(6)$ & $0.0800(8)$ & $-0.0123(6)$ & $-0.0188(6)$ & $-0.0113(5)$ \\
\hline $\mathrm{O} 8$ & $0.0344(6)$ & $0.0472(6)$ & 0.0624 & $-0.0188(5)$ & $-0.0104(5)$ & $-0.0056(5)$ \\
\hline O9 & $0.0398(7)$ & $0.1016(10)$ & $0.0954(9)$ & $-0.0460(7)$ & $-0.0199(6)$ & $-0.0011(7)$ \\
\hline $\mathrm{O} 10$ & $0.0581(8)$ & $0.0714(7)$ & $0.0565(7)$ & $-0.0242(6)$ & $0.0065(6)$ & $-0.0276(6)$ \\
\hline $\mathrm{O} 11$ & $0.0560(8)$ & $0.0537(7)$ & 0.0504 & $-0.0140(5)$ & $-0.0050(6)$ & $-0.0049(6)$ \\
\hline $\mathrm{O} 12$ & 0.048 & & & -0.015 & (6) & 0.011 \\
\hline $\mathrm{C} 1$ & $0.0419(9)$ & $0.0485(8)$ & $0.0612(8)$ & $-0.0340(6)$ & $-0.0245(6)$ & $0.0123(7)$ \\
\hline $\mathrm{C} 2$ & $0.0348(8)$ & $0.0496(8)$ & $0.0523(8)$ & $-0.0267(6)$ & $-0.0172(6)$ & $0.0077(7)$ \\
\hline C 3 & $0.0314(8)$ & $0.0526(8)$ & $0.0479(8)$ & $-0.0282(6)$ & $-0.0124(6)$ & $0.0081(7)$ \\
\hline $\mathrm{C} 4$ & $0.0337(8)$ & $0.0564(9)$ & $0.0459(8)$ & $-0.0272(6)$ & $-0.0089(7)$ & $0.0061(7)$ \\
\hline $\mathrm{C} 5$ & $0.0420(9)$ & & & & $(6)$ & \\
\hline C6 & $0.0563(11)$ & $0.0830(11)$ & 0.0525 & $-0.0421(7)$ & $-0.0199(8)$ & $0.0142(10)$ \\
\hline $\mathrm{C} 7$ & $0.0643(11)$ & $0.1103(14)$ & $0.0577(8)$ & $-0.0547(7)$ & $-0.0330(7)$ & $0.0233(11)$ \\
\hline $\mathrm{C} 8$ & $0.0684(12)$ & $0.0967(12)$ & $0.0809(9)$ & $-0.0597(7)$ & $-0.0394(8)$ & $0.0136(10)$ \\
\hline $\mathrm{C} 9$ & $0.0572(10)$ & $0.0705(9)$ & $0.0786(9)$ & $-0.0506(6)$ & $-0.0384(7)$ & $0.0141(8)$ \\
\hline $\mathrm{C} 10$ & $0.0454(9)$ & $0.0555(8)$ & $0.0587(8)$ & $-0.0374(6)$ & $-0.0224(7)$ & $0.0117(7)$ \\
\hline C11 & $0.0311(8)$ & $0.0579(9)$ & 0.0575 & $-0.0242(7)$ & $-0.0154(6)$ & $-0.0058(7)$ \\
\hline $\mathrm{C} 12$ & $0.0694(11)$ & $0.0780(12)$ & $0.0515(9)$ & $-0.0160(8)$ & $-0.0240(8)$ & $-0.0232(9)$ \\
\hline $\mathrm{C} 13$ & $0.0429(9)$ & $0.0473(9)$ & $0.0567(9)$ & $-0.0186(7)$ & $-0.0205(7)$ & $0.0010(7)$ \\
\hline C14 & $0.0934(13)$ & $0.0599(11)$ & $0.0789(11)$ & $-0.0228(8)$ & $-0.0478(8)$ & $-0.0127(9)$ \\
\hline C15 & $0.0435(9)$ & $0.0621(10)$ & $0.0373(8)$ & $-0.0212(7)$ & $-0.0112(7)$ & $0.0064(8)$ \\
\hline C16 & $0.0447(9)$ & $0.0574(10)$ & $0.0449(8)$ & $-0.0186(7)$ & $-0.0193(7)$ & $0.0062(8)$ \\
\hline C17 & $0.0444(9)$ & $0.0553(10)$ & $0.0487(9)$ & $-0.0168(7)$ & $-0.0188(7)$ & $0.0103(8)$ \\
\hline C18 & $0.0459(10)$ & $0.0504(10)$ & $0.0407(9)$ & $-0.0108(7)$ & $-0.0084(8)$ & $0.0044(9)$ \\
\hline
\end{tabular}


$\begin{array}{llllll}\mathrm{U}_{11} & \mathrm{U}_{22} & \mathrm{U}_{33} & \mathrm{U}_{23} & \mathrm{U}_{13} & \mathrm{U}_{12}\end{array}$

$\begin{array}{lllllll}\text { C19 } & 0.0392(10) & 0.0691(12) & 0.0480(10) & -0.0173(8) & 0.0017(8) & -0.0063(9)\end{array}$

$\begin{array}{lllllll}\mathrm{C} 20 & 0.0469(11) & 0.0626(11) & 0.0475(9) & -0.0251(8) & -0.0017(8) & 0.0062(9)\end{array}$

$\begin{array}{lllllll}\mathrm{C} 21 & 0.0386(9) & 0.0470(10) & 0.0449(9) & -0.0072(8) & -0.0032(8) & -0.0019(8)\end{array}$

$\begin{array}{lllllll}\mathrm{C} 22 & 0.0471(10) & 0.0458(10) & 0.0466(9) & -0.0041(8) & -0.0153(8) & 0.0004(8)\end{array}$

$\begin{array}{lllllll}\mathrm{C} 23 & 0.0433(9) & 0.0379(9) & 0.0560(10) & -0.0073(8) & -0.0150(8) & 0.0010(8)\end{array}$

$\begin{array}{lllllll}\mathrm{C} 24 & 0.0341(8) & 0.0343(8) & 0.0565(9) & -0.0108(7) & -0.0105(7) & -0.0077(7)\end{array}$

$\begin{array}{lllllll}\mathrm{C} 25 & 0.0311(8) & 0.0439(9) & 0.0616(10) & -0.0126(7) & -0.0147(7) & -0.0053(7)\end{array}$

$\begin{array}{llllllll}\mathrm{C} 26 & 0.0343(9) & 0.0441(9) & 0.0607(10) & -0.0129(8) & -0.0062(8) & -0.0043(8)\end{array}$

$\begin{array}{lllllll}\mathrm{C} 27 & 0.0351(8) & 0.0289(7) & 0.0550(9) & -0.0127(6) & -0.0178(6) & 0.0013(6)\end{array}$

$\begin{array}{lllllll}\mathrm{C} 28 & 0.0359(8) & 0.0313(7) & 0.0556(8) & -0.0166(6) & -0.0177(6) & -0.0022(6)\end{array}$

$\begin{array}{lllllll}\mathrm{C} 29 & 0.0343(8) & 0.0308(7) & 0.0528(8) & -0.0169(6) & -0.0148(6) & -0.0017(6)\end{array}$

$\begin{array}{lllllll}\mathrm{C} 30 & 0.0371(8) & 0.0332(7) & 0.0554(8) & -0.0207(6) & -0.0194(6) & 0.0018(6)\end{array}$

$\begin{array}{lllllll}\mathrm{C} 31 & 0.0351(7) & 0.0272(7) & 0.0663(9) & -0.0162(6) & -0.0284(6) & 0.0051(6)\end{array}$

$\begin{array}{lllllll}\mathrm{C} 32 & 0.0465(8) & 0.0357(8) & 0.0737(9) & -0.0190(6) & -0.0356(6) & 0.0028(7)\end{array}$

$\begin{array}{lllllll}\mathrm{C} 33 & 0.0553(9) & 0.0451(9) & 0.0921(11) & -0.0190(7) & -0.0441(7) & -0.0064(7)\end{array}$

$\begin{array}{lllllll}\text { C34 } & 0.0418(8) & 0.0398(9) & 0.0956(12) & -0.0101(8) & -0.0341(7) & -0.0092(7)\end{array}$

$\begin{array}{llllllll}\mathrm{C} 35 & 0.0370(8) & 0.0344(8) & 0.0713(11) & -0.0104(7) & -0.0201(7) & -0.0044(7)\end{array}$

$\begin{array}{lllllll}\mathrm{C} 36 & 0.0318(8) & 0.0288(7) & 0.0640(9) & -0.0139(6) & -0.0207(6) & 0.0014(6)\end{array}$

$\begin{array}{lllllll}\mathrm{C} 37 & 0.0461(9) & 0.0542(9) & 0.0539(9) & -0.0203(7) & -0.0120(7) & -0.0187(7)\end{array}$

$\begin{array}{llllllll}\mathrm{C} 38 & 0.0747(12) & 0.1607(19) & 0.0832(12) & -0.0435(11) & -0.0347(8) & -0.0431(11)\end{array}$

$\begin{array}{lllllll}\text { C39 } & 0.0421(9) & 0.0363(7) & 0.0488(8) & -0.0192(6) & -0.0112(7) & -0.0049(7)\end{array}$

$\begin{array}{lllllll}\mathrm{C} 40 & 0.0369(10) & 0.0757(13) & 0.0870(14) & -0.0222(10) & -0.0062(9) & -0.0180(9)\end{array}$

$\begin{array}{lllllll}\mathrm{C} 41 & 0.0471(9) & 0.0373(8) & 0.0546(8) & -0.0203(6) & -0.0229(6) & 0.0005(7)\end{array}$

$\begin{array}{lllllll}\mathrm{C} 42 & 0.0522(10) & 0.0409(8) & 0.0562(9) & -0.0238(6) & -0.0214(7) & 0.0104(8)\end{array}$

$\begin{array}{lllllll}\mathrm{C} 43 & 0.0511(10) & 0.0506(9) & 0.0498(9) & -0.0239(7) & -0.0149(7) & 0.0061(8)\end{array}$

$\begin{array}{lllllll}\mathrm{C} 44 & 0.0520(10) & 0.0445(8) & 0.0470(8) & -0.0207(6) & -0.0204(7) & 0.0080(8)\end{array}$

$\begin{array}{lllllll}\mathrm{C} 45 & 0.0478(9) & 0.0452(9) & 0.0581(9) & -0.0200(7) & -0.0248(7) & 0.0056(8)\end{array}$

$\begin{array}{lllllll}\mathrm{C} 46 & 0.0390(8) & 0.0421(8) & 0.0593(9) & -0.0143(7) & -0.0201(7) & -0.0027(7)\end{array}$

$\begin{array}{lllllll}\mathrm{C} 47 & 0.0516(10) & 0.0457(9) & 0.0398(8) & -0.0189(6) & -0.0106(7) & 0.0043(8)\end{array}$

$\begin{array}{lllllll}\text { C48 } & 0.0416(10) & 0.0479(10) & 0.0540(10) & -0.0141(8) & -0.0137(8) & 0.0081(8)\end{array}$

$\begin{array}{lllllll}\text { C49 } & 0.0400(9) & 0.0490(10) & 0.0520(10) & -0.0130(8) & -0.0093(8) & -0.0012(8)\end{array}$

$\begin{array}{lllllll}\mathrm{C} 50 & 0.0356(9) & 0.0457(9) & 0.0362(8) & -0.0094(7) & -0.0018(7) & -0.0004(8)\end{array}$

$\begin{array}{lllllll}\mathrm{C} 51 & 0.0554(10) & 0.0474(10) & 0.0371(8) & -0.0092(7) & -0.0150(7) & 0.0046(9)\end{array}$

$\begin{array}{lllllll}\mathrm{C} 52 & 0.0558(10) & 0.0551(9) & 0.0380(8) & -0.0195(7) & -0.0156(7) & 0.0006(8)\end{array}$

$\begin{array}{lllllll}\mathrm{C} 53 & 0.0386(9) & 0.0395(9) & 0.0373(8) & -0.0027(7) & -0.0082(7) & -0.0013(7)\end{array}$ 


\begin{tabular}{|c|c|c|c|c|c|c|}
\hline & $\mathrm{U}_{11}$ & $\mathrm{U}_{22}$ & $\mathrm{U}_{33}$ & $\mathrm{U}_{23}$ & $\mathrm{U}_{13}$ & $\mathrm{U}_{12}$ \\
\hline C54 & $0.0350(8)$ & $0.0425(9)$ & $0.0392(8)$ & $-0.0062(7)$ & $-0.0083(7)$ & $-0.0040(7)$ \\
\hline C55 & $0.0323(8)$ & $0.0373(8)$ & $0.0471(9)$ & $-0.0079(7)$ & $-0.0124(7)$ & $0.0008(7)$ \\
\hline C56 & $0.0402(9)$ & $0.0274(8)$ & $0.0577(10)$ & $-0.0024(7)$ & $-0.0165(7)$ & $0.0007(7)$ \\
\hline $\mathrm{C} 57$ & $0.0395(9)$ & $0.0322(9)$ & $0.0548(10)$ & $0.0034(8)$ & $-0.0146(8)$ & $-0.0015(7)$ \\
\hline C58 & $0.0448(10)$ & $0.0472(11)$ & $0.0720(13)$ & $0.0022(10)$ & $-0.0159(9)$ & $-0.0090(9)$ \\
\hline C59 & $0.0414(11)$ & $0.0584(13)$ & $0.0841(15)$ & $0.0080(12)$ & $-0.0119(10)$ & $-0.0119(10)$ \\
\hline C60 & $0.0371(11)$ & $0.0612(13)$ & $0.0594(13)$ & $0.0100(11)$ & $0.0058(10)$ & $-0.0026(10)$ \\
\hline C61 & $0.0418(10)$ & $0.0483(10)$ & $0.0440(10)$ & $0.0021(9)$ & 0.0003(9) & $0.0019(9)$ \\
\hline C62 & $0.0343(9)$ & $0.0366(9)$ & $0.0436(9)$ & $0.0042(8)$ & $-0.0084(7)$ & $0.0014(8)$ \\
\hline C63 & $0.0500(10)$ & $0.0736(10)$ & $0.0544(9)$ & $-0.0350(7)$ & $0.0016(8)$ & $83(8)$ \\
\hline C64 & $0.0840(17)$ & $0.1195(16)$ & $0.133(2)$ & $-0.0665(13)$ & $0.0311(16)$ & $-0.0556(13)$ \\
\hline C65 & $0.0393(8)$ & $0.0444(9)$ & $0.0508(9)$ & $-0.0157(7)$ & $-0.0164(7)$ & $-0.0022(7)$ \\
\hline C66 & $0.0555(13)$ & $0.0763(13)$ & $0.0817(14)$ & $-0.0353(10)$ & $-0.0111(11)$ & $0.0219(11)$ \\
\hline C67 & $0.0423(9)$ & $0.0364(8)$ & $0.0733(11)$ & $-0.0106(8)$ & $-0.0204(8)$ & $58(7)$ \\
\hline C68 & $0.0452(10)$ & $0.0633(11)$ & $0.0767(12)$ & $-0.0254(9)$ & $-0.0240(8)$ & $0.0088(9)$ \\
\hline C69 & $0.0438(10)$ & $0.0686(12)$ & $0.0813(12)$ & & & $37(9)$ \\
\hline $\mathrm{C} 70$ & $0.0401(8)$ & $0.0381(8)$ & $0.0832(10)$ & $-0.0201(7)$ & $-0.0286(7)$ & $-0.0065(6)$ \\
\hline C71 & $0.0608(11)$ & $0.0321(8)$ & $0.0899(12)$ & $-0.0227(7)$ & $-0.0339(8)$ & $0.0039(8)$ \\
\hline $\mathrm{C} 72$ & $0.0621(11)$ & $0.0341(9)$ & $0.0815(12)$ & $-0.0158(8)$ & $-0.0320(9)$ & $0.0037(8)$ \\
\hline $\mathrm{C} 73$ & $0.0372(8)$ & $0.0333(7)$ & $0.0852(10)$ & $-0.0236(6)$ & $-0.0304(6)$ & $-0.0029(6)$ \\
\hline C74 & $0.0352(8)$ & $0.0420(8)$ & $0.0816(11)$ & $-0.0224(7)$ & $-0.0224(7)$ & $-0.0011(7)$ \\
\hline $\mathrm{C} 75$ & $0.0418(8)$ & $0.0408(8)$ & $0.0776(10)$ & $-0.0233(7)$ & $-0.0330(7)$ & $0.0036(7)$ \\
\hline C76 & $0.0444(8)$ & $0.0448(8)$ & $0.0720(8)$ & $-0.0323(6)$ & $-0.0307(6)$ & $0.0023(6)$ \\
\hline $\mathrm{C} 77$ & $0.0555(10)$ & $0.0469(8)$ & $0.0870(10)$ & $-0.0395(7)$ & $-0.0336(8)$ & $0.0120(8)$ \\
\hline C78 & $0.0564(10)$ & $0.0364(8)$ & $0.0875(10)$ & $-0.0287(7)$ & $-0.0382(7)$ & $0.0067(7)$ \\
\hline C79 & $0.365(5)$ & $0.219(6)$ & $.111(3)$ & $0.063(3)$ & $-0.164(3)$ & $-0.187(4)$ \\
\hline $\mathrm{C} 80$ & $0.092(4)$ & $0.139(4)$ & $0.107(3)$ & $-0.081(2)$ & $-0.019(3)$ & $0.005(3)$ \\
\hline $\mathrm{C} 81$ & $0.088(3)$ & $24(8)$ & $0.103(4)$ & $-0.022(4)$ & $-0.041(3)$ & $-0.045(4)$ \\
\hline $\mathrm{C} 82$ & $0.461(12)$ & $0.266(8)$ & $0.064(4)$ & $-0.023(4)$ & $-0.047(5)$ & $-0.214(6)$ \\
\hline $\mathrm{C} 83$ & $0.175(4)$ & $0.175(5)$ & $0.077(3)$ & $0.000(3)$ & $-0.055(2)$ & $-0.107(3)$ \\
\hline $\mathrm{C} 84$ & $0.153(4)$ & $0.170(6)$ & $0.237(5)$ & $-0.080(4)$ & $-0.135(3)$ & $0.011(4)$ \\
\hline $79^{\prime}$ & $0.233(7)$ & $0.192(10)$ & $0.686(16)$ & $-0.106(9)$ & $-0.303(6)$ & $0.000(6)$ \\
\hline C80' & $0.276(8)$ & $0.214(8)$ & $0.109(4)$ & $-0.050(5)$ & $-0.116(4)$ & $0.135(6)$ \\
\hline C81' & $0.103(5)$ & $0.227(5)$ & $0.212(4)$ & $-0.175(3)$ & $-0.022(4)$ & $-0.004(4)$ \\
\hline C82' & $0.220(5)$ & $0.245(6)$ & $0.098(3)$ & $0.013(4)$ & $-0.085(3)$ & $-0.147(4)$ \\
\hline
\end{tabular}




$\begin{array}{ccccccc} & \mathrm{U}_{11} & \mathrm{U}_{22} & \mathrm{U}_{33} & \mathrm{U}_{23} & \mathrm{U}_{13} & \mathrm{U}_{12} \\ \mathrm{C} 83^{\prime} & 0.237(9) & 0.221(9) & 0.232(12) & -0.030(8) & -0.001(9) & -0.140(6) \\ \mathrm{C} 84^{\prime} & 0.166(6) & 0.170(5) & 0.138(4) & -0.092(3) & -0.065(3) & -0.010(4) \\ \mathrm{C} 85 & 0.057(2) & 0.081(3) & 0.060(2) & -0.0316(17) & -0.0272(16) & 0.009(2) \\ \mathrm{C} 86 & 0.136(4) & 0.060(3) & 0.202(6) & -0.036(3) & -0.071(4) & -0.018(3) \\ \text { C87 } & 0.097(3) & 0.104(3) & 0.069(2) & -0.046(2) & -0.037(2) & 0.035(3) \\ \text { C88 } & 0.188(5) & 0.206(6) & 0.279(5) & -0.160(3) & -0.168(3) & 0.064(4) \\ \text { C89 } & 0.221(3) & 0.049(2) & 0.146(3) & 0.0016(19) & -0.1412(19) & -0.039(2) \\ \text { C90 } & 0.068(4) & 0.093(3) & 0.273(7) & -0.101(3) & 0.045(4) & 0.001(3) \\ \text { C85' } & 0.077(4) & 0.081(4) & 0.147(6) & 0.004(4) & 0.006(4) & 0.020(3) \\ \text { C86' } & 0.083(4) & 0.165(6) & 0.055(3) & -0.020(3) & -0.005(3) & 0.021(4) \\ \text { C87' } & 0.058(3) & 0.085(3) & 0.129(4) & -0.061(2) & 0.002(3) & 0.016(2) \\ \text { C88' } & 0.093(5) & 0.462(13) & 0.168(6) & 0.212(6) & 0.055(5) & 0.114(6) \\ \text { C89' } & 0.095(4) & 0.166(5) & 0.061(3) & -0.043(3) & -0.017(3) & 0.043(4) \\ \text { C90' } & 0.042(2) & 0.124(3) & 0.087(2) & -0.0690(17) & -0.0130(17) & -0.004(2) \\ \text { C91 } & 0.094(3) & 0.095(2) & 0.179(4) & -0.070(2) & -0.030(3) & 0.016(2) \\ \text { C11 } & 0.0765(6) & 0.0929(6) & 0.1375(8) & -0.0406(5) & -0.0219(5) & -0.0137(5) \\ \text { C12 } & 0.1312(8) & 0.1206(6) & 0.1140(6) & -0.0731(4) & 0.0004(6) & -0.0427(5) \\ \text { C91' } & 0.036(4) & 0.318(7) & 0.239(6) & -0.243(4) & -0.006(4) & 0.003(5) \\ \text { C11' } & 0.290(4) & 0.180(2) & 0.209(3) & -0.107(2) & 0.090(3) & -0.140(2) \\ \text { C12' } & 0.0604(13) & 0.350(4) & 0.209(2) & -0.1729(18) & -0.0428(12) & -0.0067(17)\end{array}$


Table 8. Hydrogen atom coordinates and isotropic atomic displacement parameters $\left(\AA^{2}\right)$ for $14\left(\mathrm{C}_{78} \mathrm{H}_{54} \mathrm{O}_{12} \cdot 2 \mathrm{C}_{6} \mathrm{H}_{12} \cdot \mathrm{CH}_{2} \mathrm{Cl}_{2}\right)$.

$\begin{array}{ccccc} & \mathrm{x} / \mathrm{a} & \mathrm{y} / \mathrm{b} & \mathrm{z} / \mathrm{c} & \mathrm{U}(\mathrm{eq}) \\ \mathrm{H} 6 & 0.7610 & 0.5798 & 1.4255 & 0.072 \\ \mathrm{H} 7 & 0.8758 & 0.6343 & 1.4567 & 0.085 \\ \mathrm{H} 8 & 0.9399 & 0.7704 & 1.3748 & 0.086 \\ \text { H9 } & 0.8885 & 0.8535 & 1.2623 & 0.071 \\ \text { H12A } & 0.6512 & 0.8446 & 0.9721 & 0.096 \\ \text { H12B } & 0.7668 & 0.8642 & 0.9407 & 0.096 \\ \text { H12C } & 0.6826 & 0.9329 & 0.9732 & 0.096 \\ \text { H14A } & 0.5011 & 0.5689 & 1.1841 & 0.106 \\ \text { H14B } & 0.5998 & 0.5433 & 1.1241 & 0.106 \\ \text { H14C } & 0.5383 & 0.6408 & 1.0987 & 0.106 \\ \text { H16 } & 0.8161 & 0.5109 & 1.3126 & 0.059 \\ \text { H17 } & 0.8016 & 0.3754 & 1.3069 & 0.061 \\ \text { H19 } & 0.4992 & 0.4238 & 1.3676 & 0.067 \\ \text { H20 } & 0.5142 & 0.5610 & 1.3718 & 0.066 \\ \text { H22 } & 0.7581 & 0.2274 & 1.3264 & 0.06 \\ \text { H23 } & 0.7941 & 0.1700 & 1.2266 & 0.059 \\ \text { H25 } & 0.5625 & 0.3339 & 1.1455 & 0.056 \\ \text { H26 } & 0.5241 & 0.3888 & 1.2466 & 0.059 \\ \text { H32 } & 0.6693 & 0.2496 & 0.8732 & 0.058 \\ \text { H33 } & 0.5397 & 0.1613 & 0.9461 & 0.071 \\ \text { H34 } & 0.4970 & 0.1132 & 1.0825 & 0.07 \\ \text { H35 } & 0.5802 & 0.1577 & 1.1460 & 0.058 \\ \text { H38A } & 1.0335 & 0.3471 & 1.1130 & 0.146 \\ \text { H38B } & 1.0542 & 0.2449 & 1.1673 & 0.146 \\ \text { H38C } & 0.9575 & 0.3069 & 1.1964 & 0.146 \\ \text { H40A } & 1.1196 & 0.4124 & 0.8446 & 0.103 \\ \text { H40B } & 1.1661 & 0.3482 & 0.9166 & 0.103 \\ \text { H40C } & 1.0943 & 0.4376 & 0.9214 & 0.103 \\ \text { H42 } & 0.9481 & 0.3074 & 0.7945 & 0.058 \\ \text { H4 } & 0.9900 & 0.4281 & 0.6802 & 0.06 \\ \text { H1 } & 0.7078 & 0.5496 & 0.7280 & 0.058\end{array}$




\begin{tabular}{|c|c|c|c|c|}
\hline & $\mathrm{x} / \mathrm{a}$ & $\mathrm{y} / \mathrm{b}$ & $\mathrm{z} / \mathrm{c}$ & $\mathrm{U}(\mathrm{eq})$ \\
\hline H46 & 0.6662 & 0.4291 & 0.8427 & 0.056 \\
\hline H48 & 1.0194 & 0.5729 & 0.6837 & 0.061 \\
\hline H49 & 1.0479 & 0.7205 & 0.6465 & 0.06 \\
\hline H51 & 0.8109 & 0.8031 & 0.5498 & 0.059 \\
\hline H52 & 0.7820 & 0.6549 & 0.5875 & 0.059 \\
\hline $\mathrm{H} 58$ & 1.1149 & 1.0499 & 0.6182 & 0.073 \\
\hline H59 & 1.2517 & 1.0131 & 0.5282 & 0.086 \\
\hline H60 & 1.2416 & 0.9119 & 0.4725 & 0.081 \\
\hline H61 & 1.0958 & 0.8464 & 0.5074 & 0.065 \\
\hline H64A & 0.5867 & 0.7918 & 0.7693 & 0.168 \\
\hline H64B & 0.6374 & 0.6904 & 0.7991 & 0.168 \\
\hline $\mathrm{H} 64 \mathrm{C}$ & 0.6280 & 0.7370 & 0.7107 & 0.168 \\
\hline H66A & 0.5609 & 1.0428 & 0.7834 & 0.113 \\
\hline H66B & 0.5844 & 1.1450 & 0.7509 & 0.113 \\
\hline H66C & 0.6267 & 1.0728 & 0.8225 & 0.113 \\
\hline H68 & 1.0708 & 0.9317 & 0.7535 & 0.074 \\
\hline H69 & 1.0616 & 0.9233 & 0.8777 & 0.077 \\
\hline H71 & 0.8284 & 1.1145 & 0.8661 & 0.07 \\
\hline $\mathrm{H} 72$ & 0.8379 & 1.1243 & 0.7411 & 0.07 \\
\hline H74 & 1.0153 & 0.8732 & 0.9962 & 0.062 \\
\hline H75 & 0.9458 & 0.8006 & 1.1266 & 0.059 \\
\hline H77 & 0.7183 & 0.9999 & 1.1234 & 0.068 \\
\hline H78 & 0.7921 & 1.0751 & 0.9937 & 0.066 \\
\hline H79A & 0.5314 & 0.9661 & 0.4428 & 0.264 \\
\hline H79B & 0.6430 & 0.9793 & 0.3863 & 0.264 \\
\hline H80A & 0.6155 & 0.8742 & 0.3316 & 0.126 \\
\hline H80B & 0.5405 & 0.9665 & 0.3129 & 0.126 \\
\hline H81A & 0.4347 & 0.8481 & 0.3790 & 0.169 \\
\hline H81B & 0.4230 & 0.8984 & 0.4386 & 0.169 \\
\hline H82A & 0.4475 & 0.7334 & 0.4994 & 0.3 \\
\hline H82B & 0.5610 & 0.7365 & 0.4445 & 0.3 \\
\hline H83A & 0.5496 & 0.7538 & 0.5702 & 0.163 \\
\hline H83B & 0.4830 & 0.8488 & 0.5403 & 0.163 \\
\hline H84A & 0.6759 & 0.8091 & 0.4403 & 0.201 \\
\hline H84B & 0.6708 & 0.8586 & 0.4996 & 0.201 \\
\hline
\end{tabular}




\begin{tabular}{|c|c|c|c|c|}
\hline & $\mathrm{x} / \mathrm{a}$ & $\mathrm{y} / \mathrm{b}$ & $\mathrm{z} / \mathrm{c}$ & $\mathrm{U}(\mathrm{eq})$ \\
\hline $\mathrm{H} 79 \mathrm{C}$ & 0.6248 & 0.9883 & 0.4006 & 0.412 \\
\hline H79D & 0.6758 & 0.8944 & 0.3888 & 0.412 \\
\hline H80C & 0.5379 & 0.9593 & 0.3242 & 0.254 \\
\hline H80D & 0.4616 & 0.9515 & 0.4080 & 0.254 \\
\hline $\mathrm{H} 81 \mathrm{C}$ & 0.5863 & 0.7937 & 0.3817 & 0.187 \\
\hline H81D & 0.4722 & 0.8182 & 0.3715 & 0.187 \\
\hline H82C & 0.4262 & 0.8382 & 0.5028 & 0.216 \\
\hline H82D & 0.4466 & 0.7361 & 0.5057 & 0.216 \\
\hline H83C & 0.6272 & 0.7427 & 0.5007 & 0.289 \\
\hline H83D & 0.5479 & 0.7444 & 0.5822 & 0.289 \\
\hline $\mathrm{H} 84 \mathrm{C}$ & 0.6275 & 0.8805 & 0.5268 & 0.169 \\
\hline H84D & 0.5125 & 0.9064 & 0.5220 & 0.169 \\
\hline H85A & 0.3876 & 0.6494 & 0.4377 & 0.077 \\
\hline H85B & 0.2935 & 0.6175 & 0.5111 & 0.077 \\
\hline H86A & 0.2587 & 0.7730 & 0.4388 & 0.153 \\
\hline H86B & 0.2773 & 0.7509 & 0.3611 & 0.153 \\
\hline H87A & 0.1145 & 0.6898 & 0.4970 & 0.106 \\
\hline H87B & 0.1007 & 0.7717 & 0.4183 & 0.106 \\
\hline H88A & 0.1694 & 0.6706 & 0.3472 & 0.225 \\
\hline H88B & 0.0658 & 0.6411 & 0.4109 & 0.225 \\
\hline H89A & 0.1655 & 0.5434 & 0.5024 & 0.148 \\
\hline H89B & 0.1814 & 0.5121 & 0.4298 & 0.148 \\
\hline H90A & 0.3306 & 0.5876 & 0.3661 & 0.187 \\
\hline H90B & 0.3406 & 0.5103 & 0.4479 & 0.187 \\
\hline H85C & 0.1527 & 0.7684 & 0.3970 & 0.155 \\
\hline H85D & 0.1090 & 0.7345 & 0.3459 & 0.155 \\
\hline $\mathrm{H} 86 \mathrm{C}$ & 0.2726 & 0.6370 & 0.3286 & 0.139 \\
\hline H86D & 0.2840 & 0.7430 & 0.2840 & 0.139 \\
\hline $\mathrm{H} 87 \mathrm{C}$ & 0.2977 & 0.7419 & 0.4123 & 0.112 \\
\hline H87D & 0.3957 & 0.6916 & 0.3679 & 0.112 \\
\hline $\mathrm{H} 88 \mathrm{C}$ & 0.3430 & 0.5719 & 0.4873 & 0.467 \\
\hline H88D & 0.2851 & 0.5579 & 0.4321 & 0.467 \\
\hline H89C & 0.1713 & 0.5954 & 0.5551 & 0.14 \\
\hline H89D & 0.1924 & 0.6975 & 0.4984 & 0.14 \\
\hline H90C & 0.1478 & 0.5790 & 0.4355 & 0.091 \\
\hline
\end{tabular}




$\begin{array}{lcccc} & \mathrm{x} / \mathrm{a} & \mathrm{y} / \mathrm{b} & \mathrm{z} / \mathrm{c} & \mathrm{U}(\mathrm{eq}) \\ \text { H90D } & 0.0553 & 0.6375 & 0.4759 & 0.091 \\ \text { H91A } & 0.2236 & 0.4455 & 0.0994 & 0.145 \\ \text { H91B } & 0.2348 & 0.4720 & 0.0082 & 0.145 \\ \text { H91C } & 0.2350 & 0.4372 & 0.1135 & 0.194 \\ \text { H91D } & 0.2454 & 0.4936 & 0.0216 & 0.194\end{array}$

\section{References Cited}

S1. APEX2 is a Bruker AXS crystallographic software package for single crystal data collection, reduction and preparation.

S2. Sheldrick, G. M., SHELXL-2013, Crystallographic software package, Bruker AXS, Inc., Madison, Wisconsin, USA.

S3. $\mathrm{R}_{1}=\sum\left(\| \mathrm{F}_{\mathrm{o}}|-| \mathrm{F}_{\mathrm{c}} \mid\right) / \sum\left|\mathrm{F}_{\mathrm{o}}\right|, \mathrm{wR}_{2}=\left[\sum\left[\mathrm{w}\left(\mathrm{F}_{\mathrm{o}}^{2}-\mathrm{F}_{\mathrm{c}}^{2}\right)^{2}\right] / \sum\left[\mathrm{w}\left(\mathrm{F}_{\mathrm{o}}^{2}\right)^{2}\right]\right]^{1 / 2}, \mathrm{R}_{\text {int. }}=\sum \mid \mathrm{F}_{\mathrm{o}}^{2}-$ $\left.\mathrm{F}_{\mathrm{o}}^{2}($ mean $)\right|^{2} / \sum\left[\mathrm{F}_{\mathrm{o}}^{2}\right]$, and GOF $=\left[\sum\left[\mathrm{w}\left(\mathrm{F}_{\mathrm{o}}^{2}-\mathrm{F}_{\mathrm{c}}^{2}\right)^{2}\right] /(\mathrm{n}-\mathrm{p})\right]^{1 / 2}$, where $\mathrm{n}$ is the number of reflections and $\mathrm{p}$ is the total number of parameters which were varied during the last refinement cycle.

S4. International Tables for X-ray Crystallography (1974). Vol. IV, p. 55. Birmingham: Kynoch Press. (Present distributor, D. Reidel, Dordrecht.). 


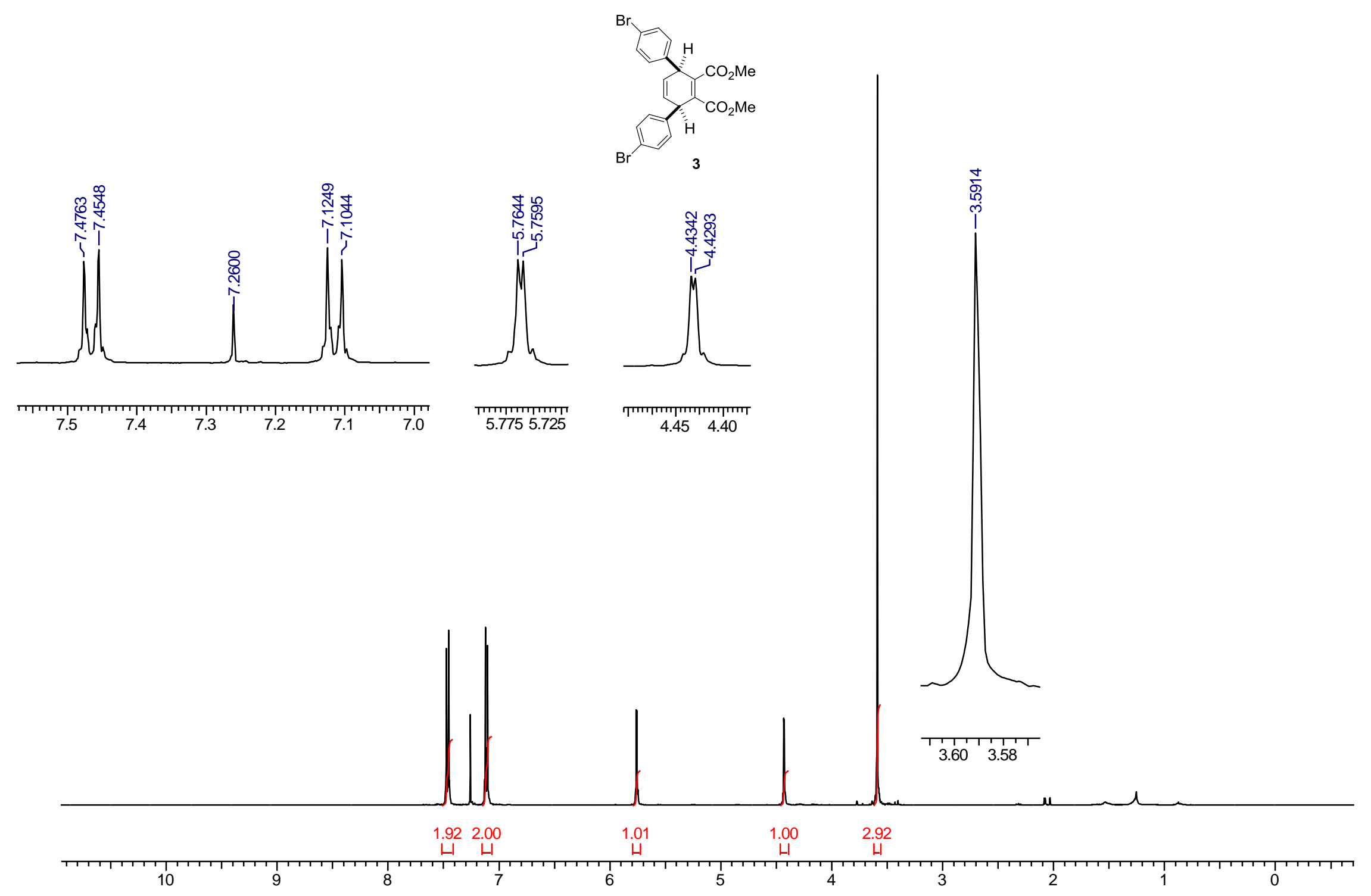




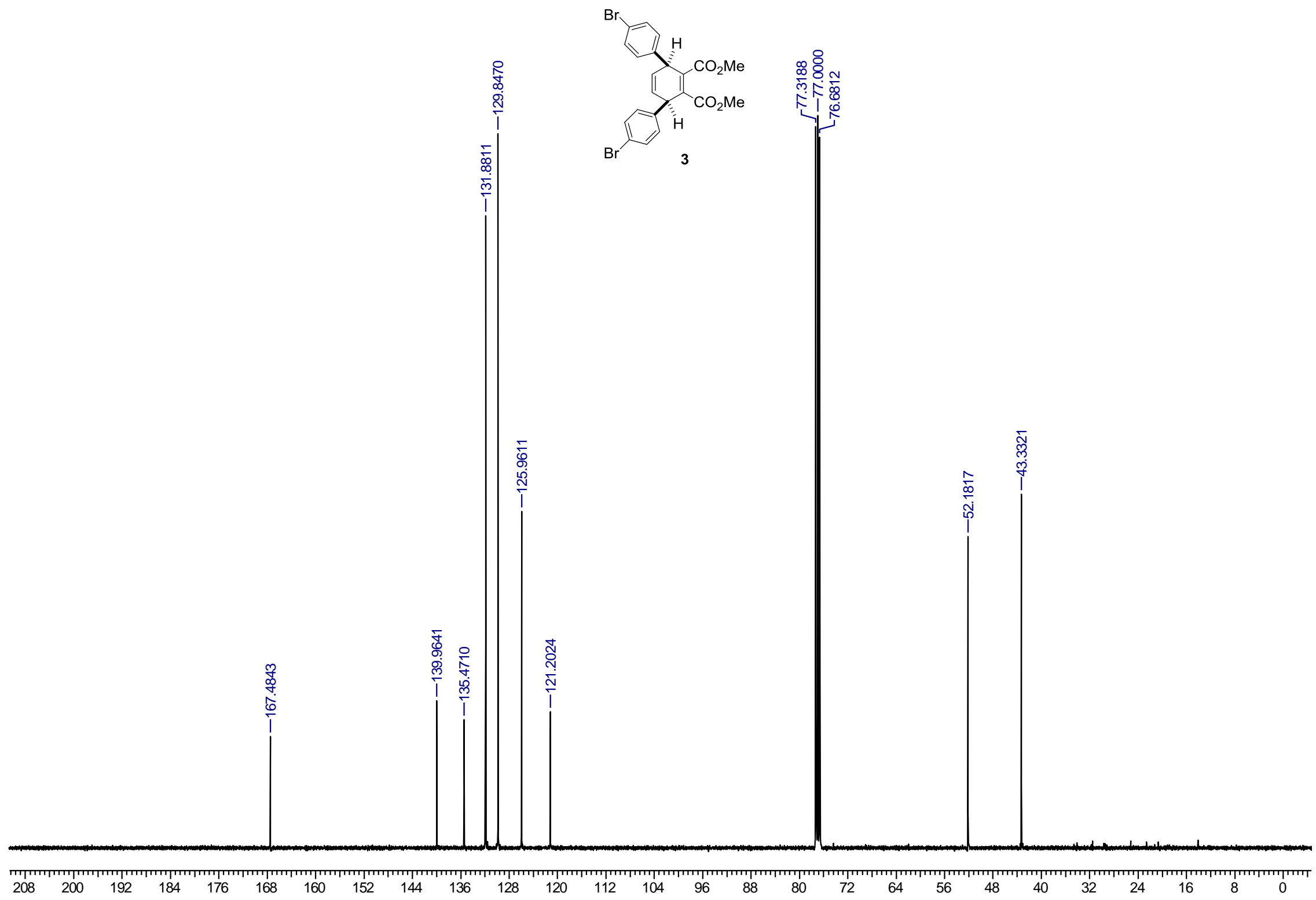

S47 


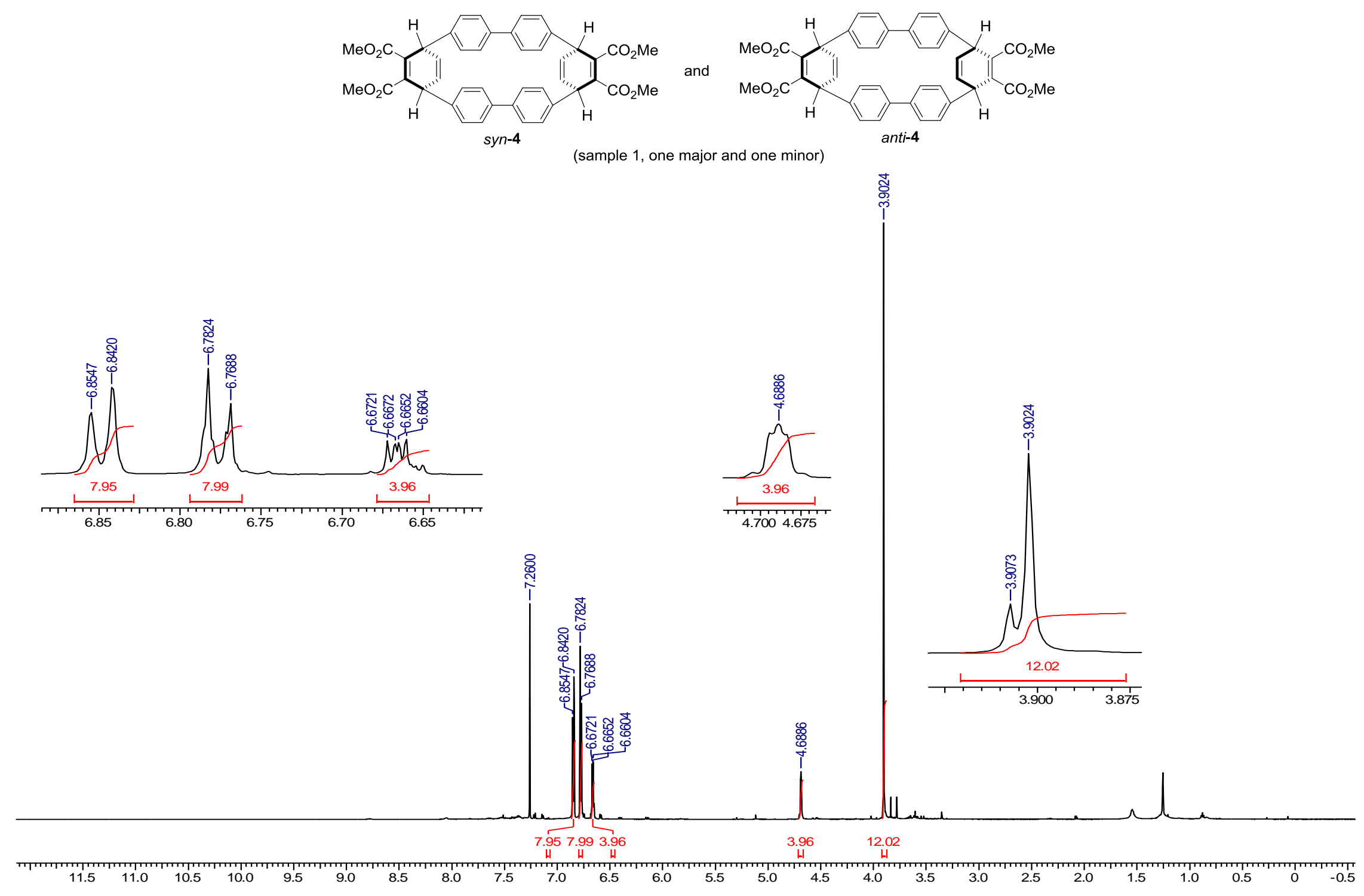




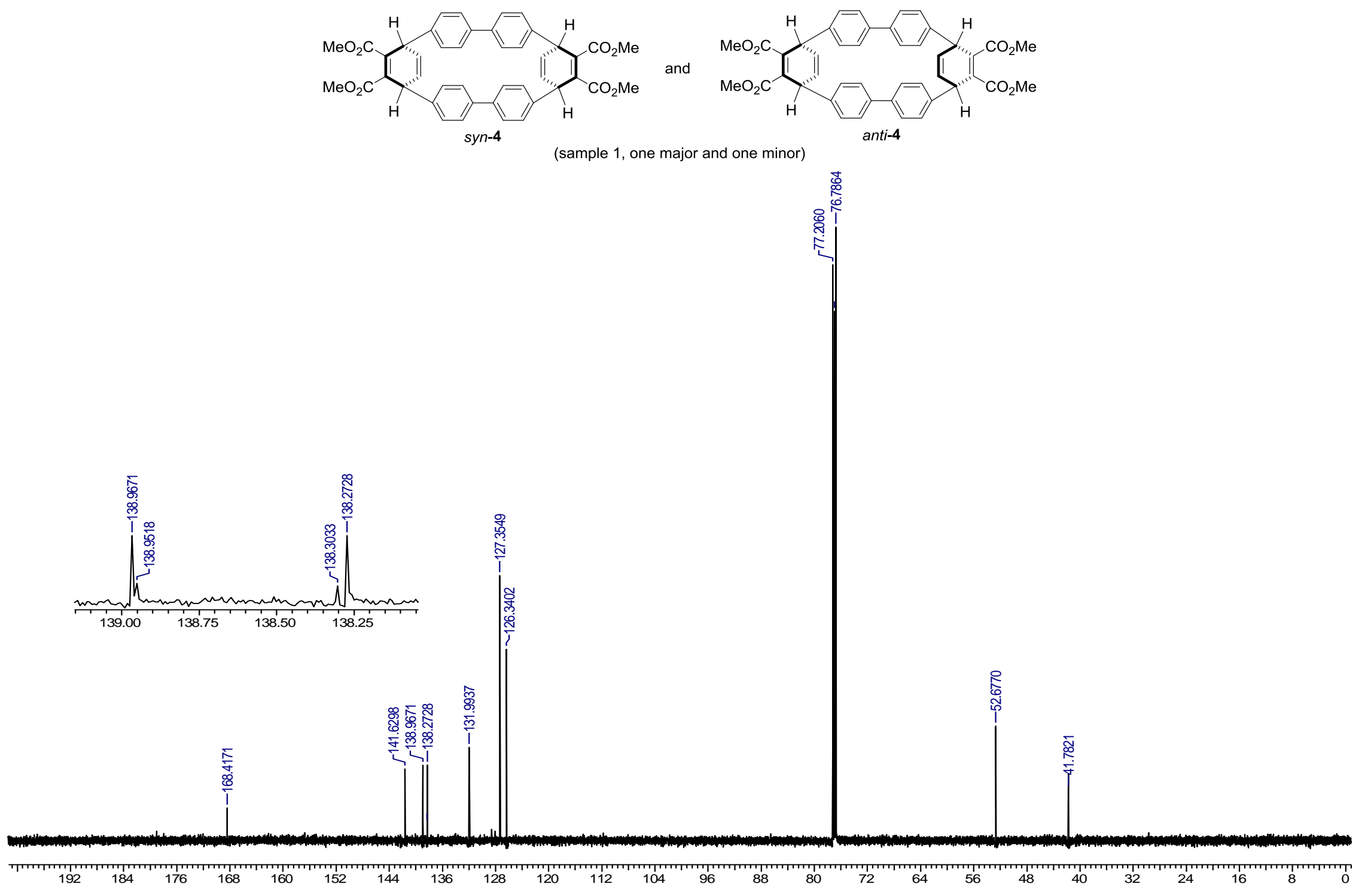




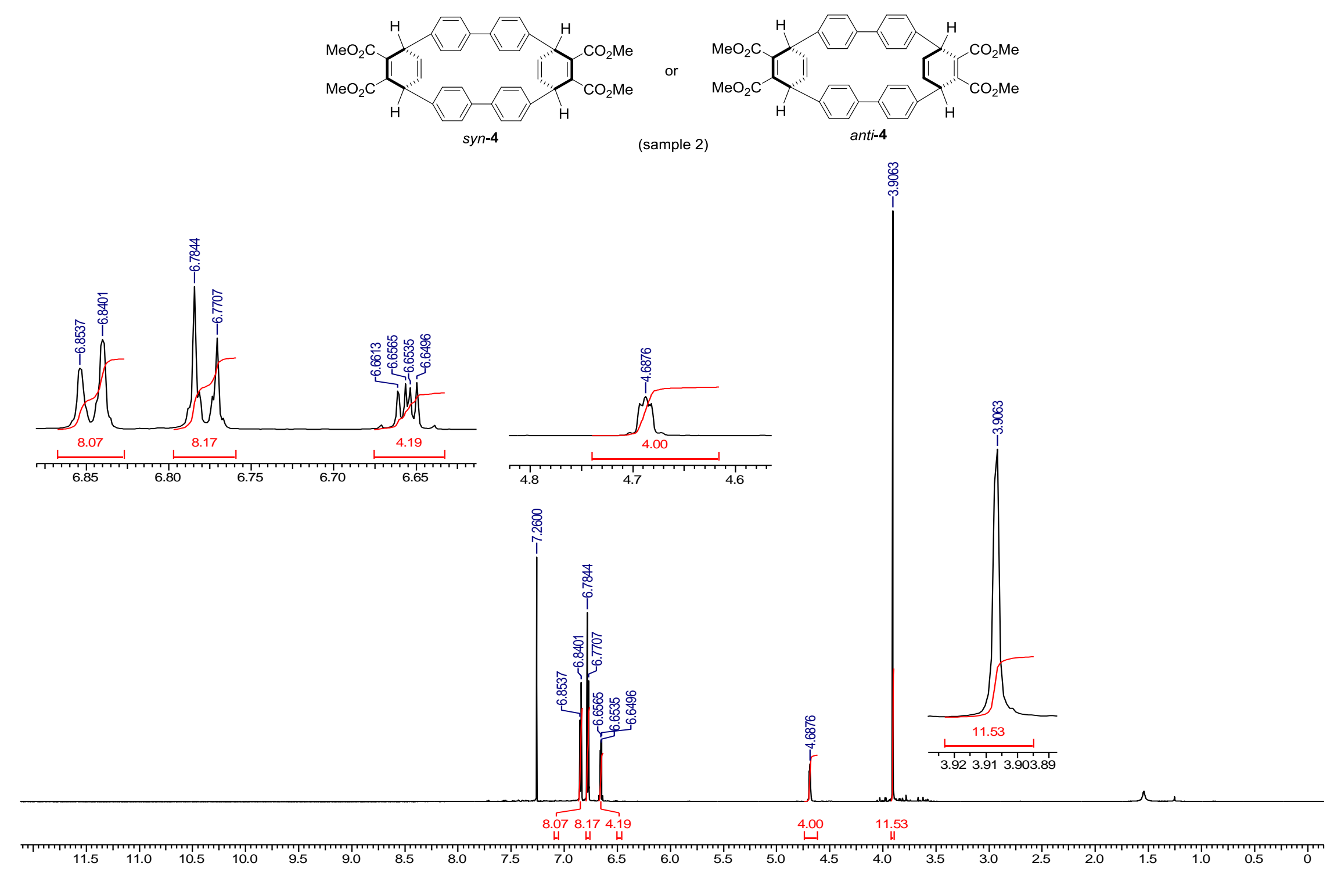



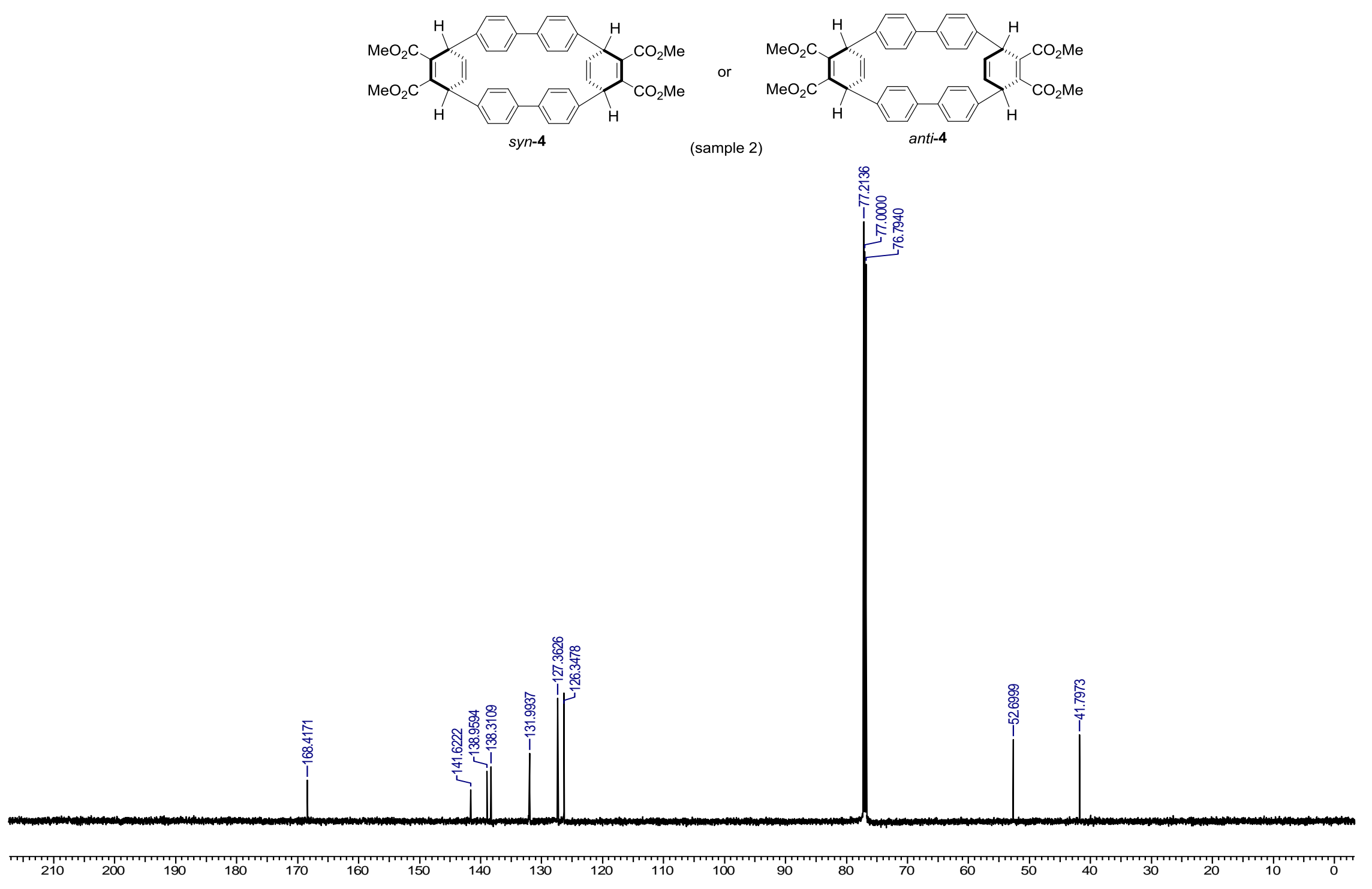


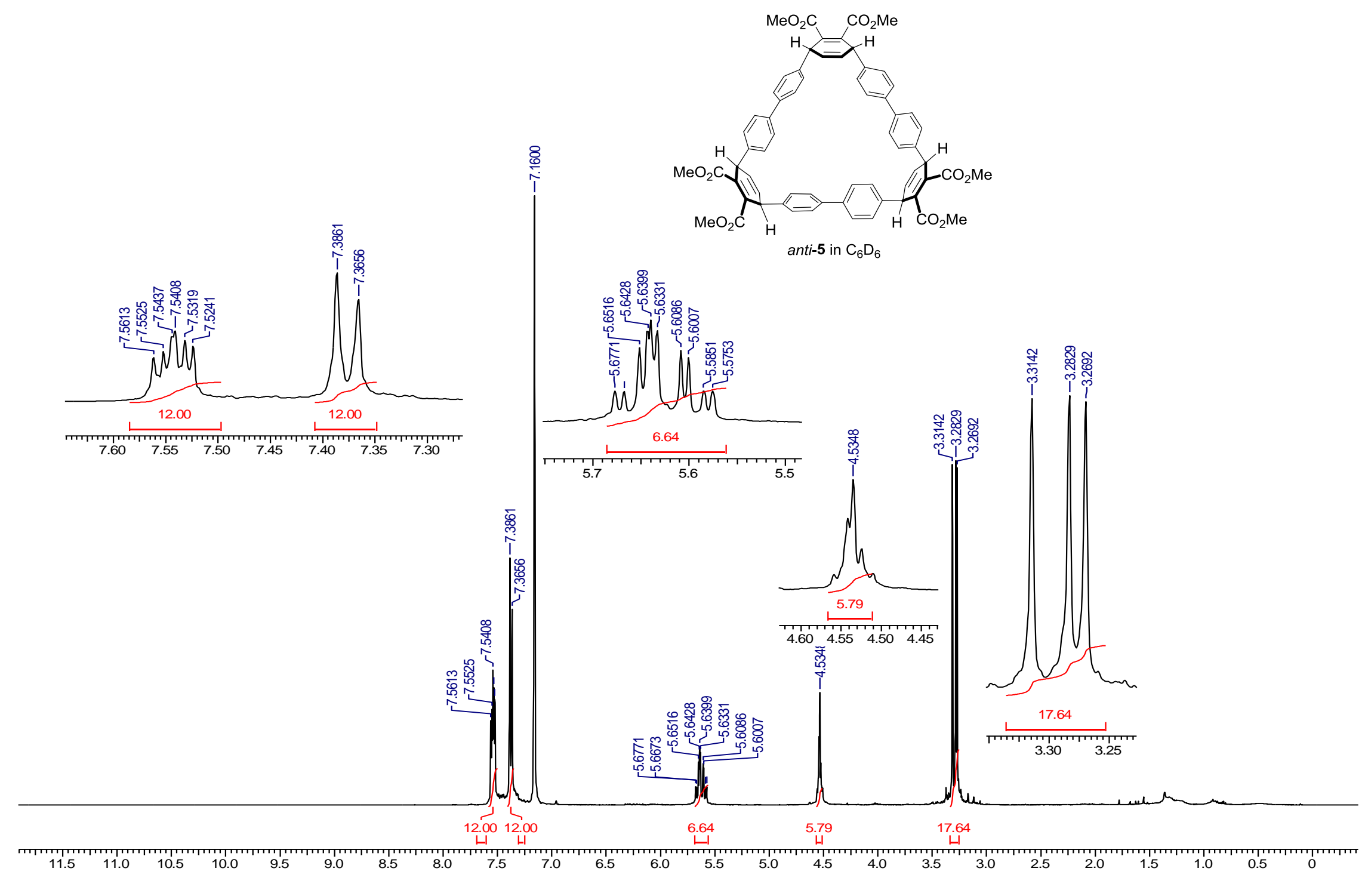




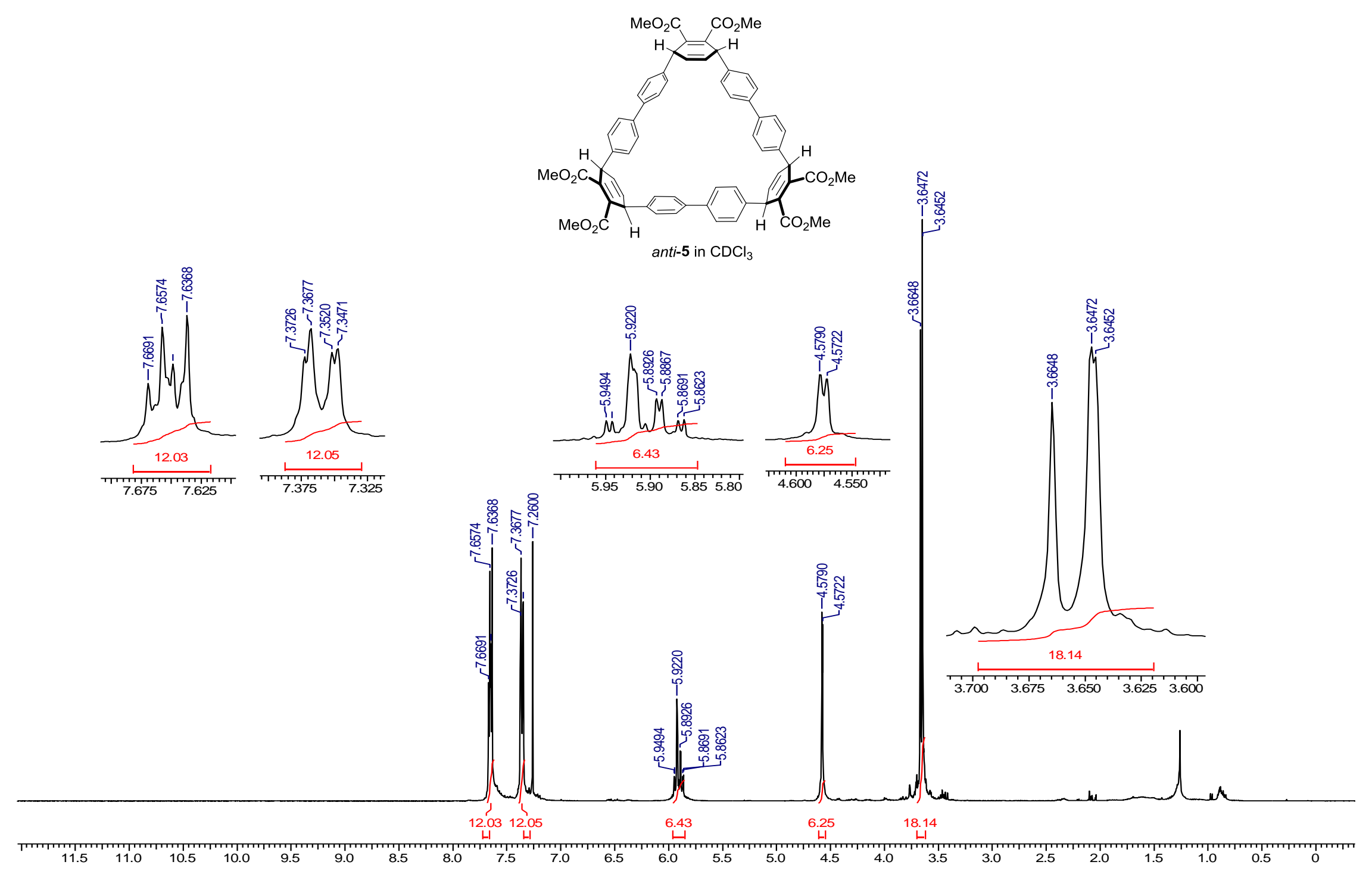




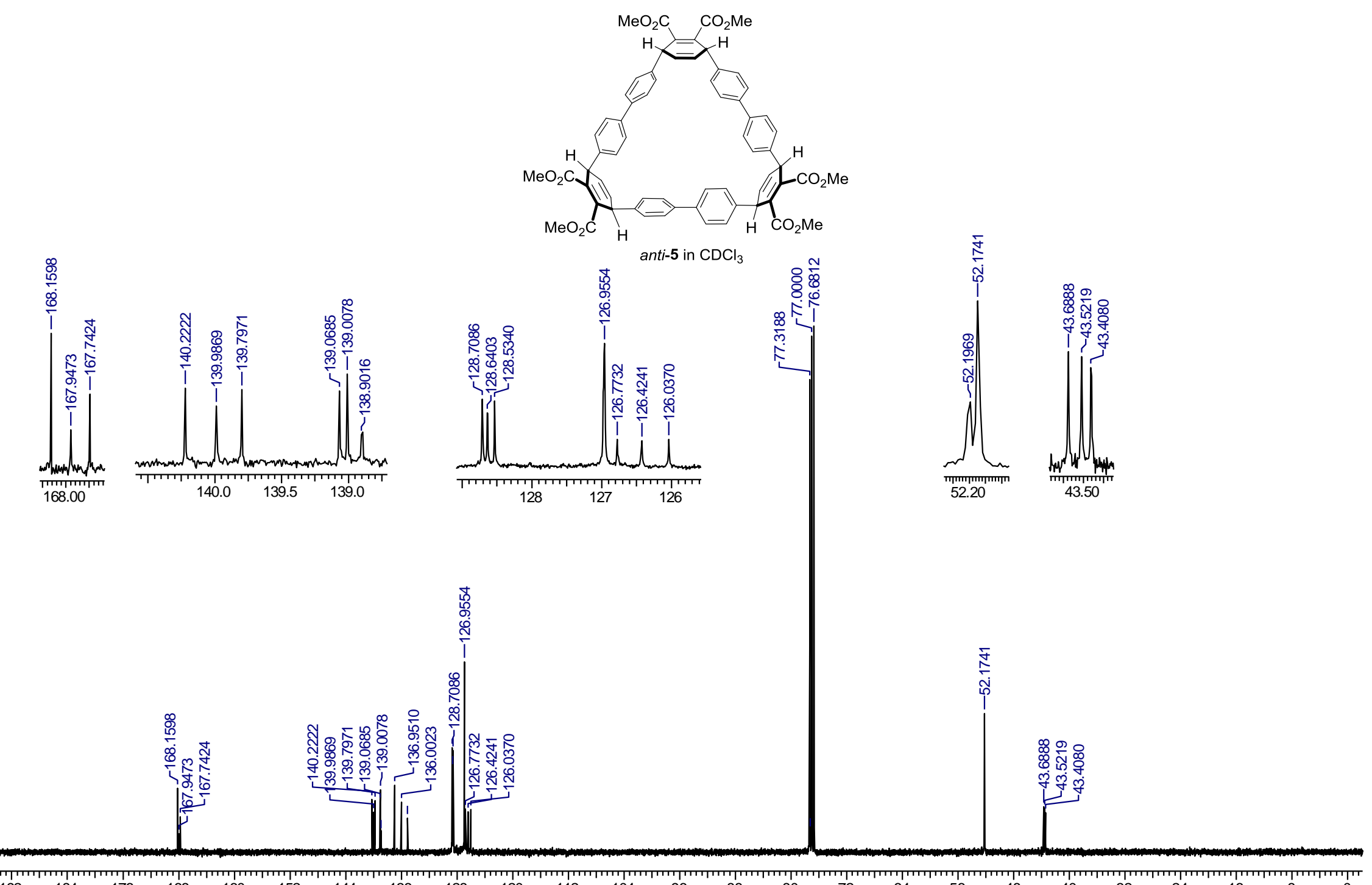

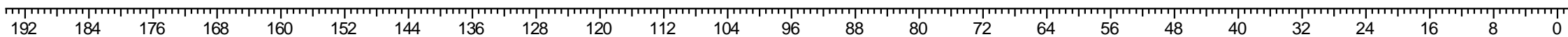




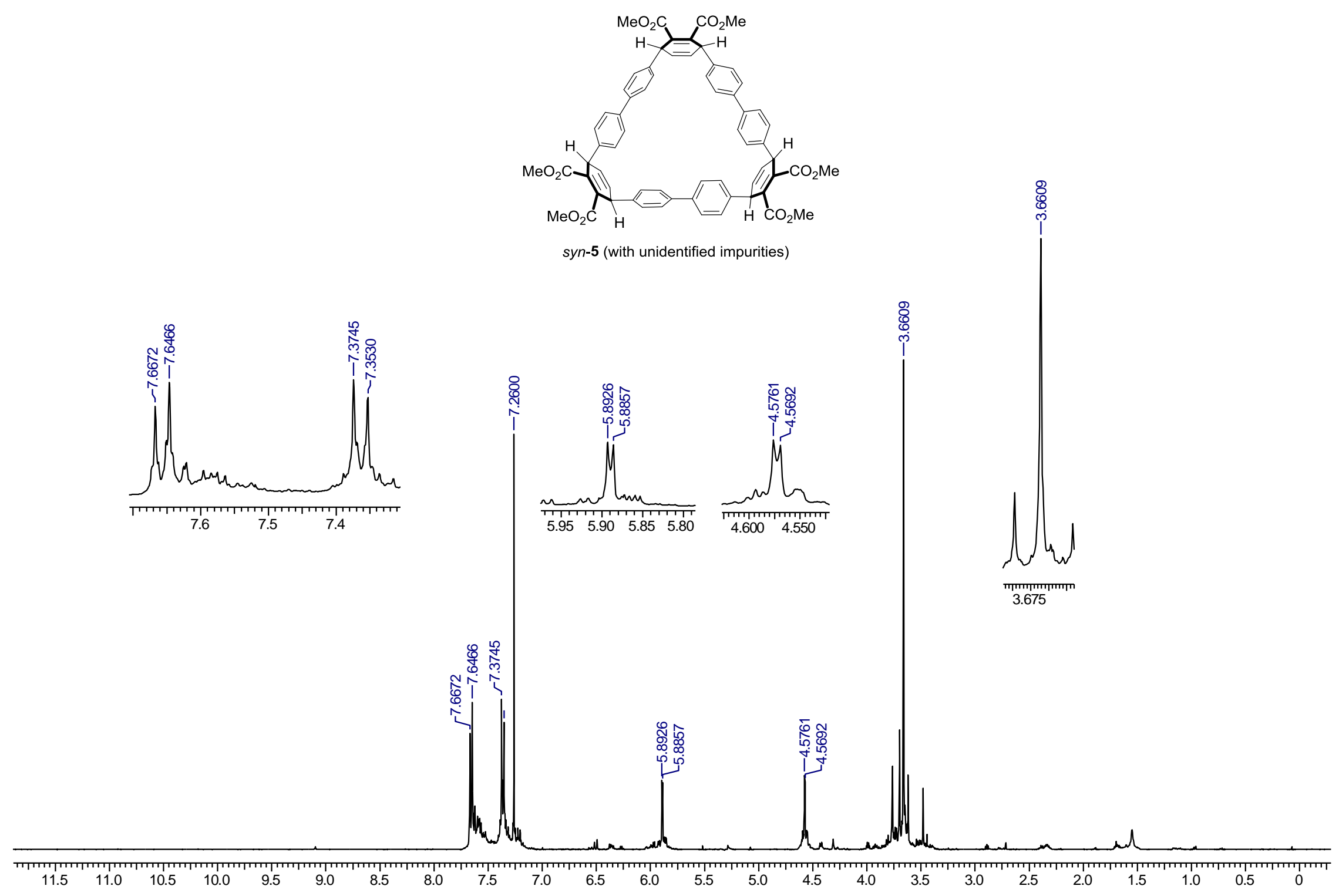




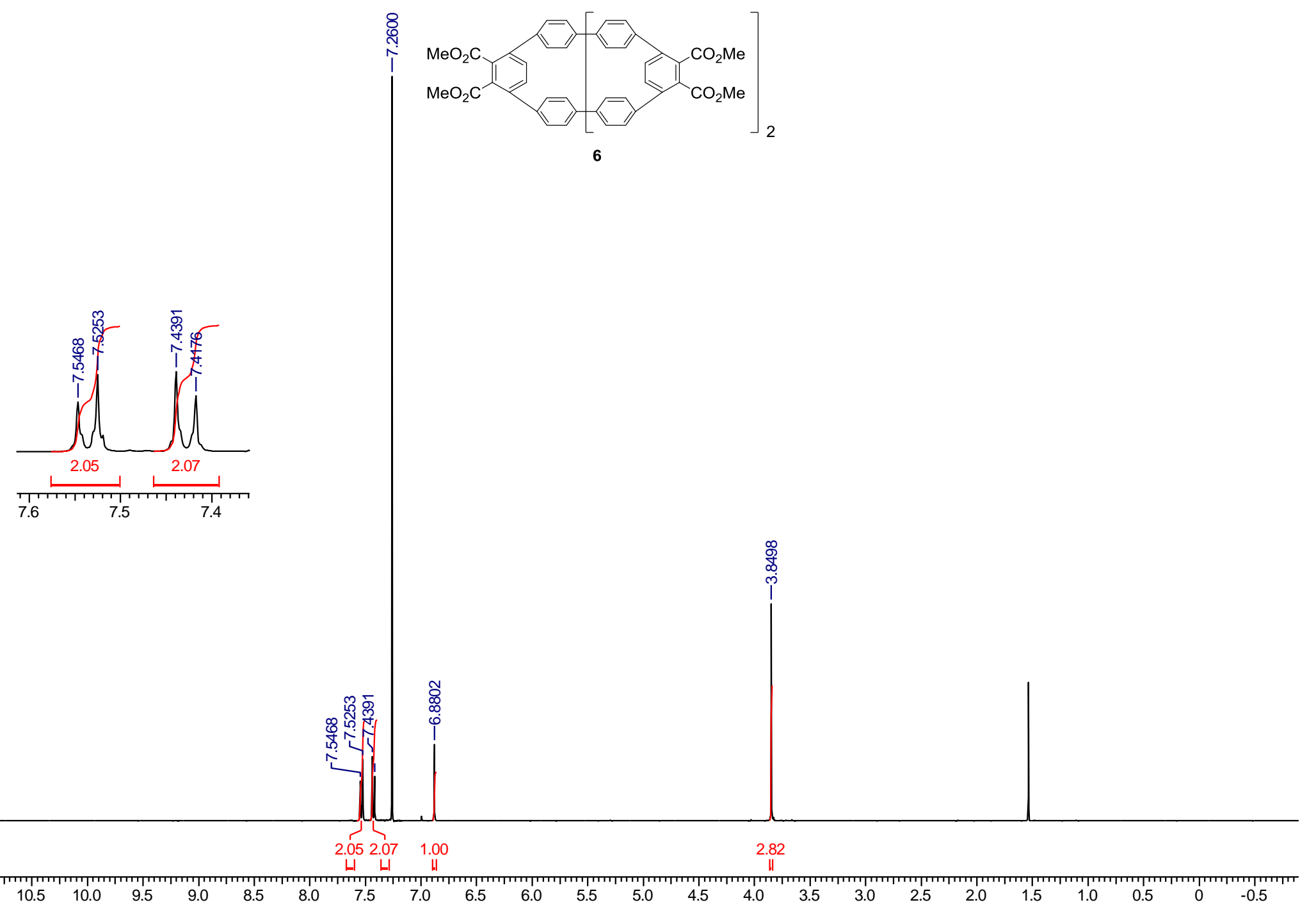



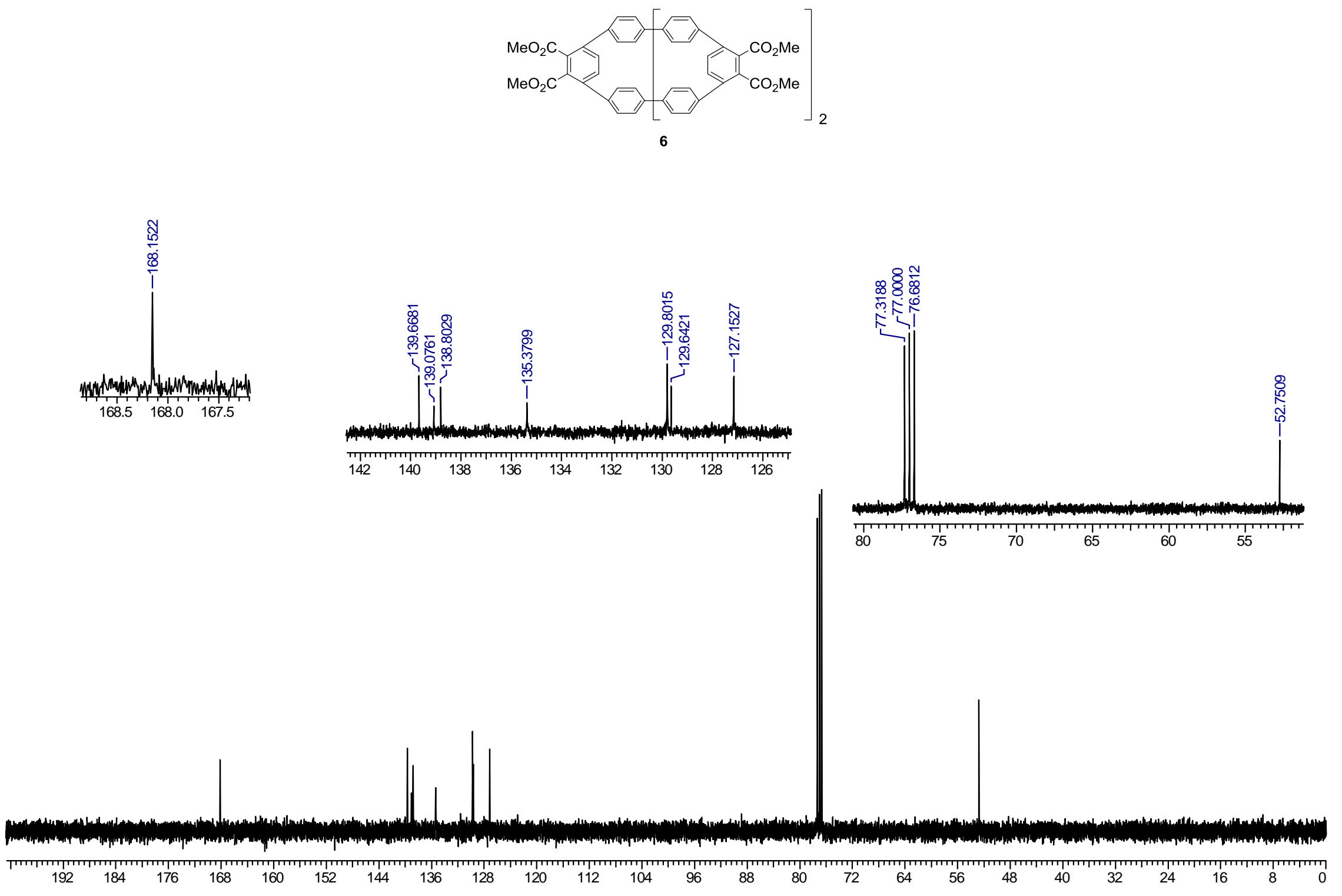


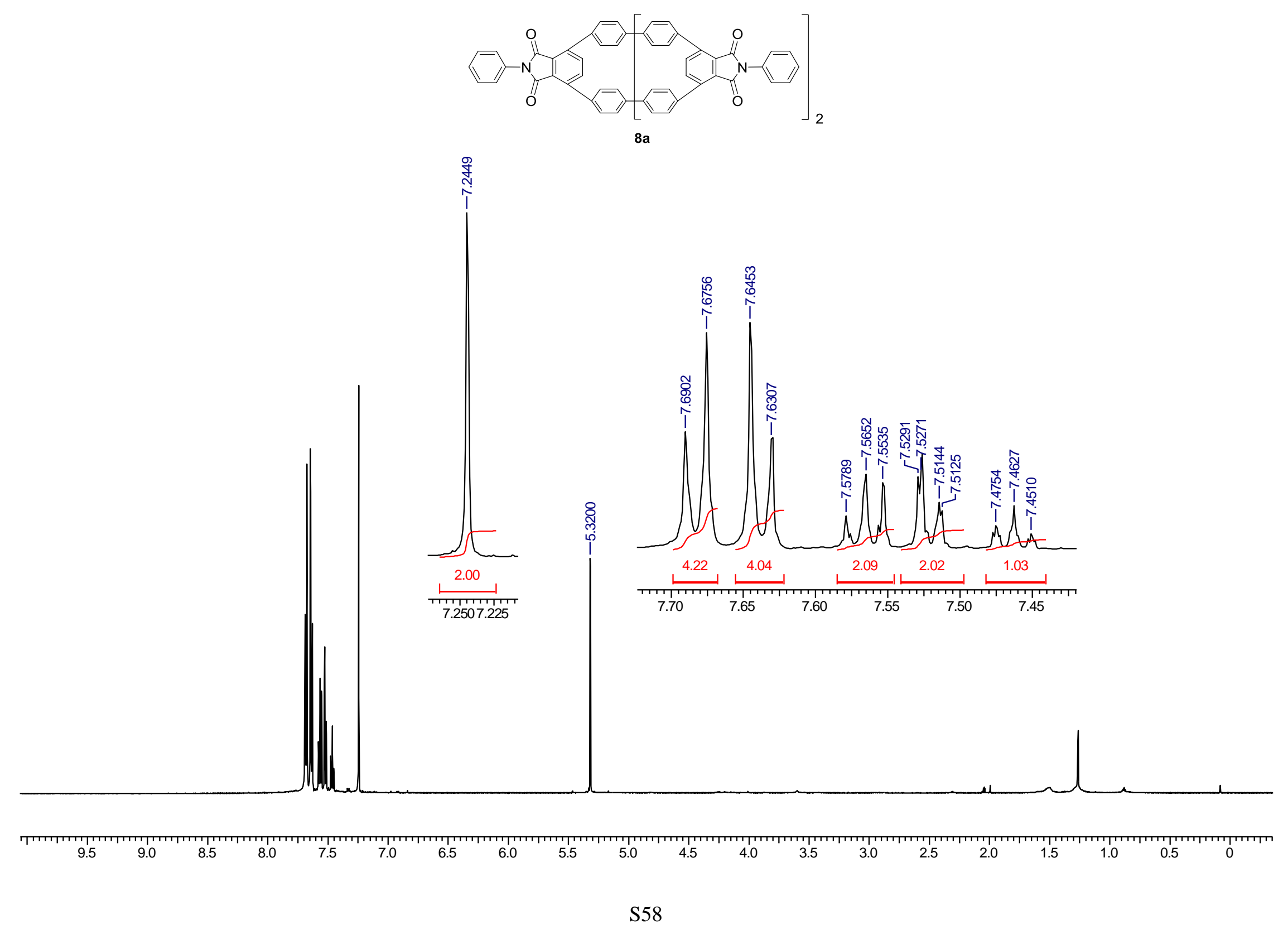



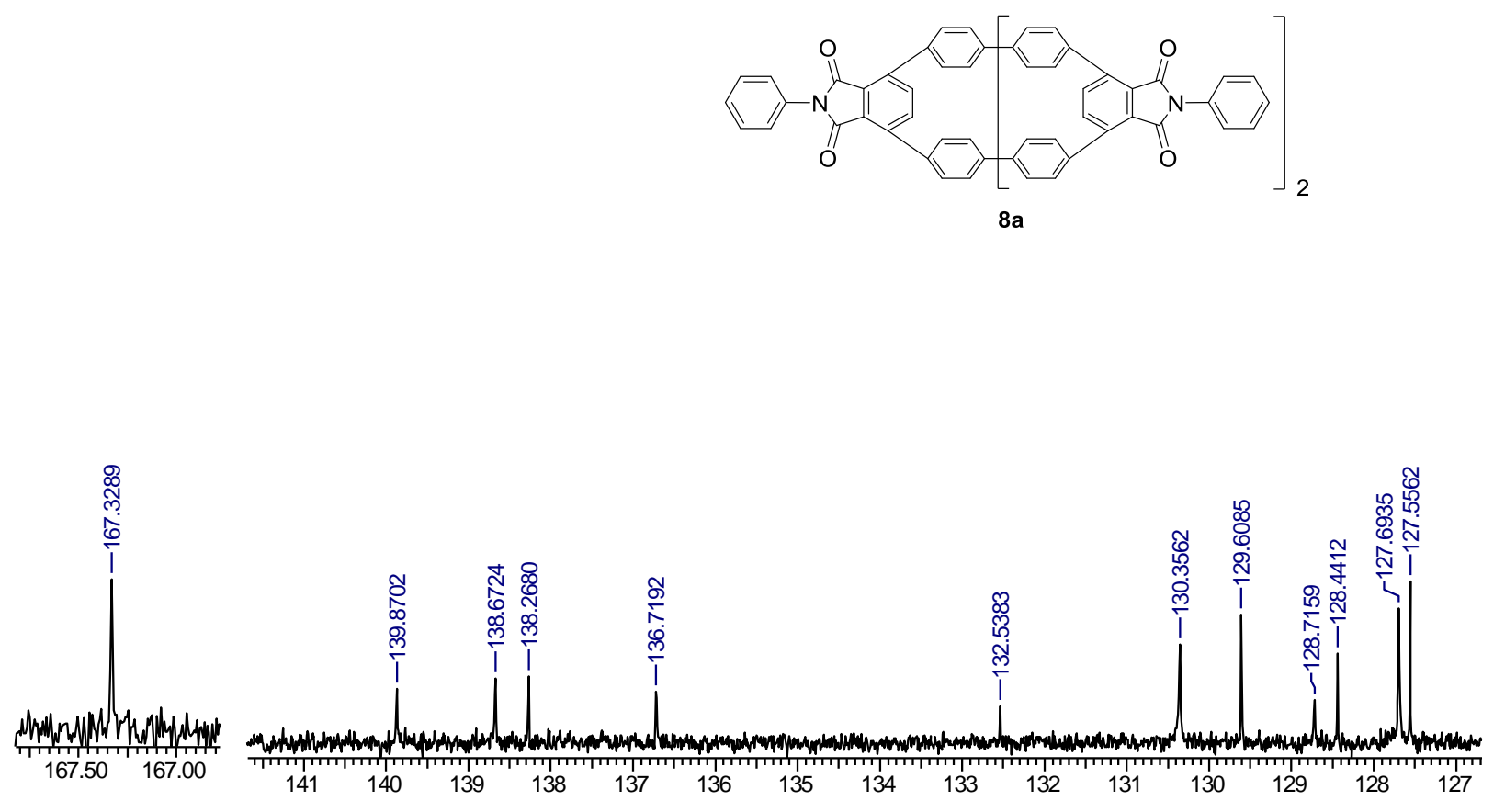


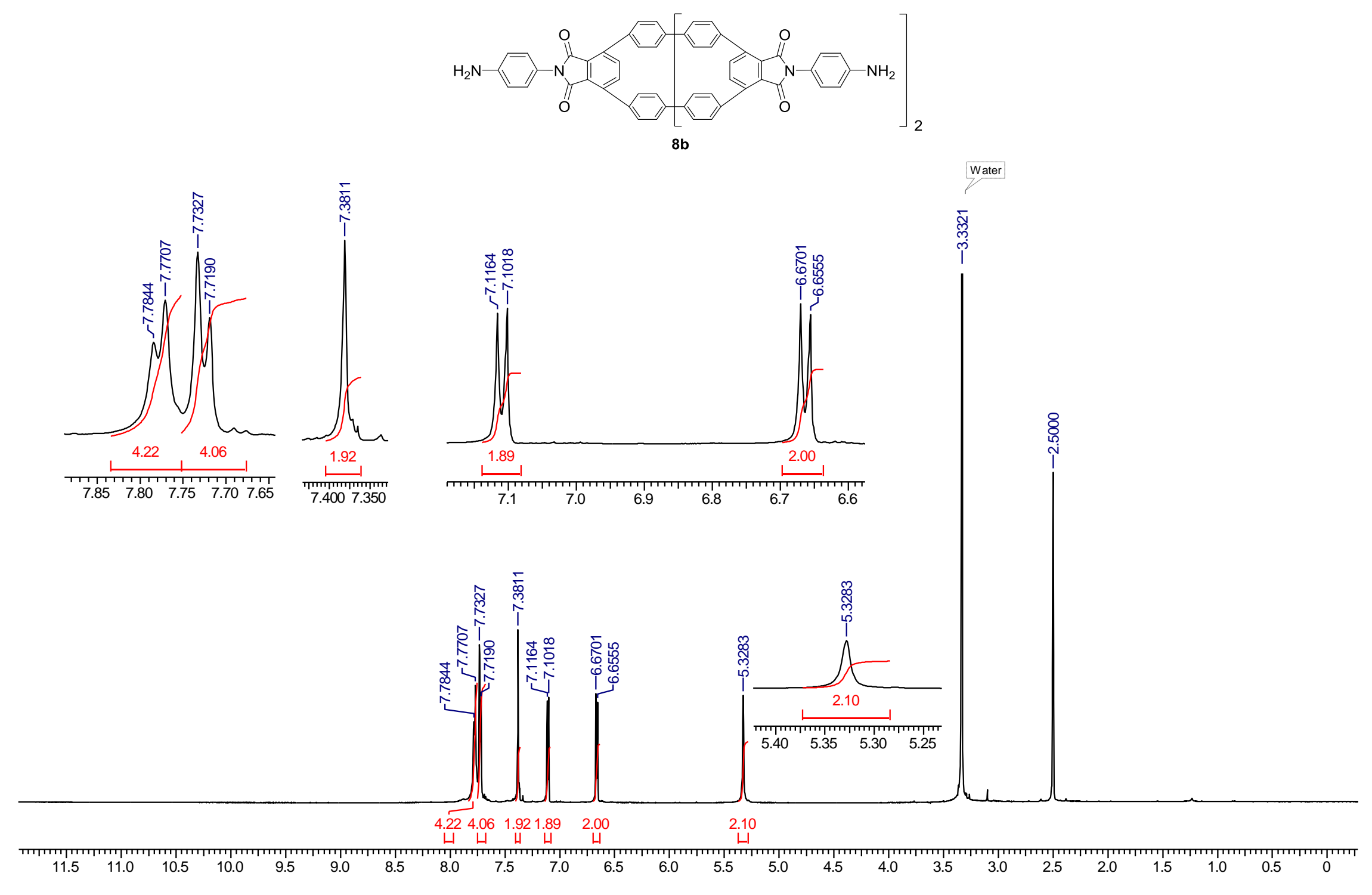



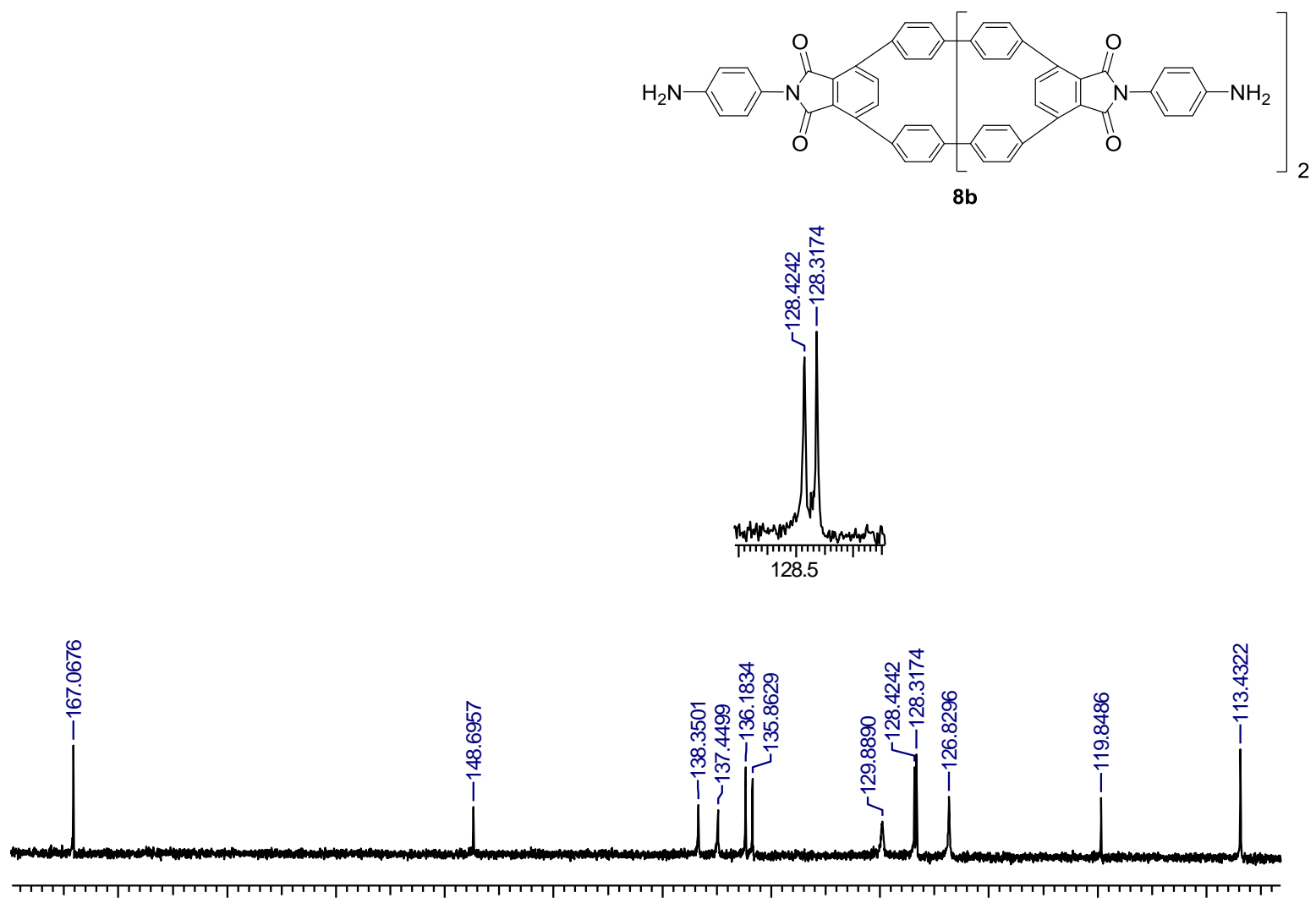

165

160

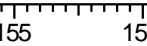

$45 \quad 140$

135

$130 \quad 125$

$120 \quad 115$

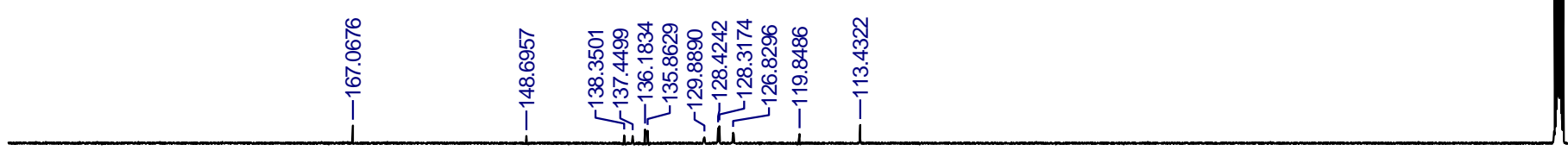

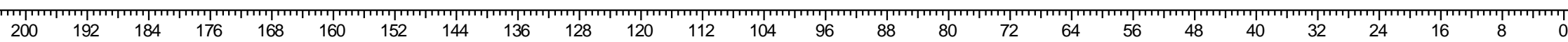




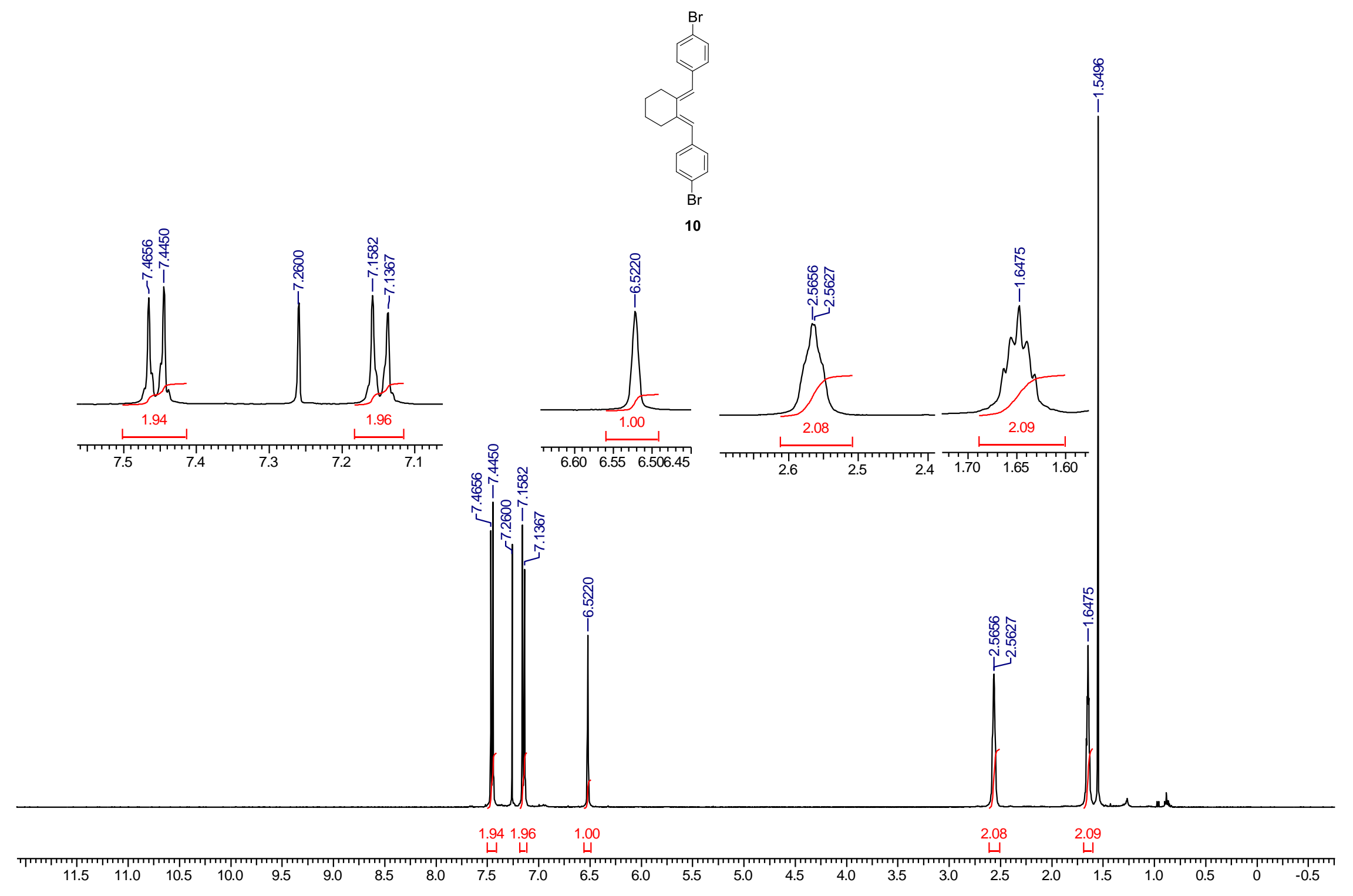




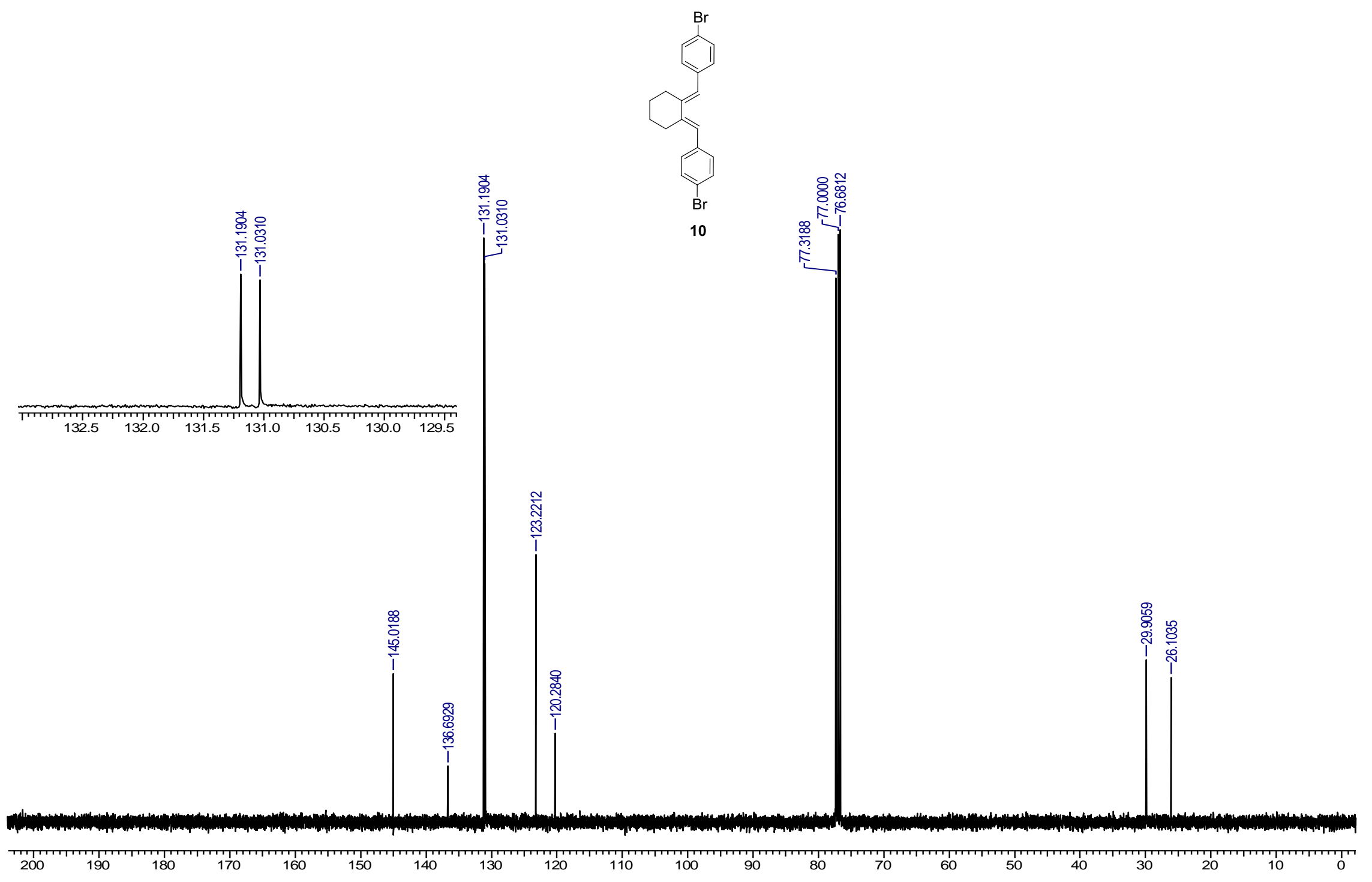



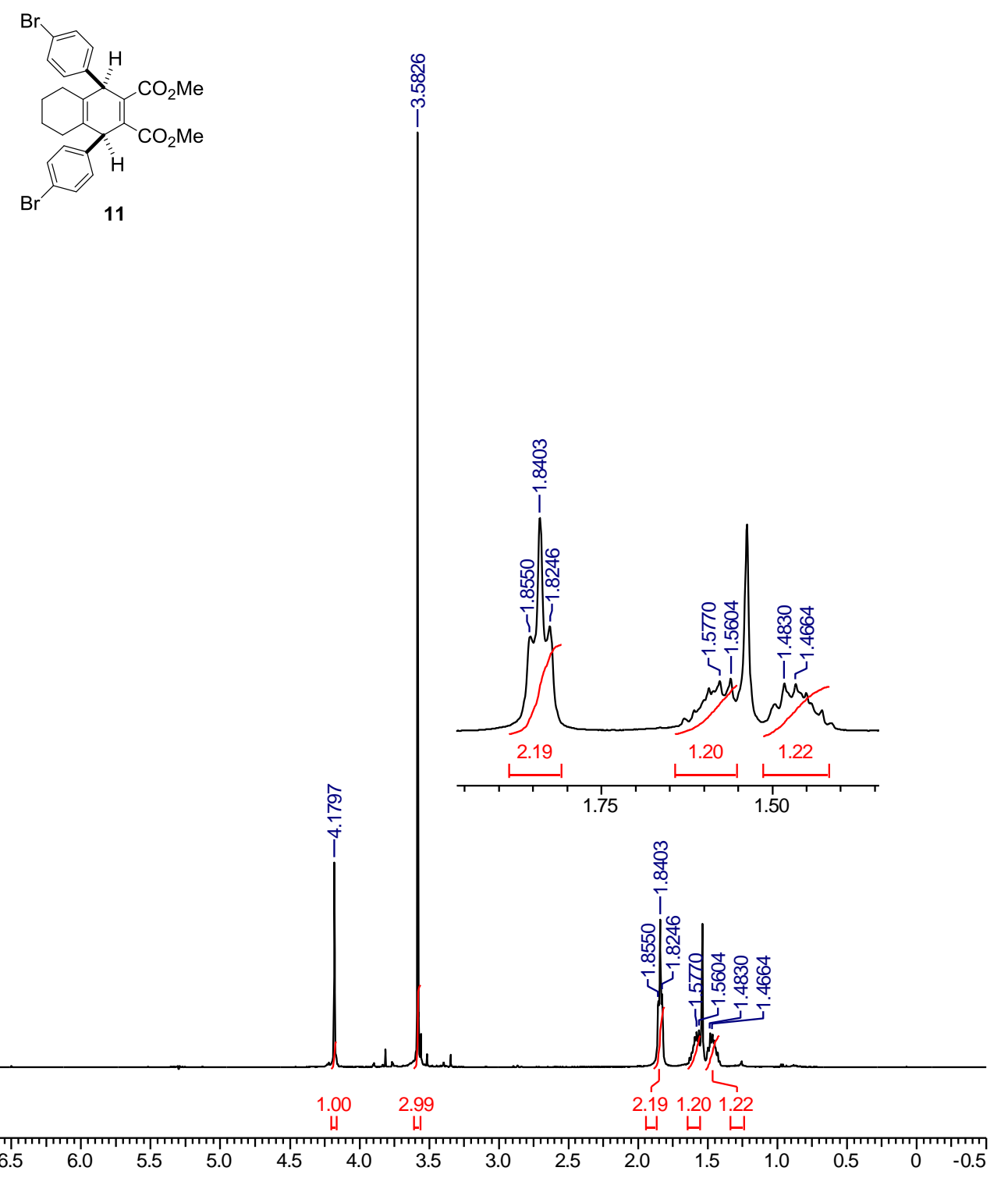

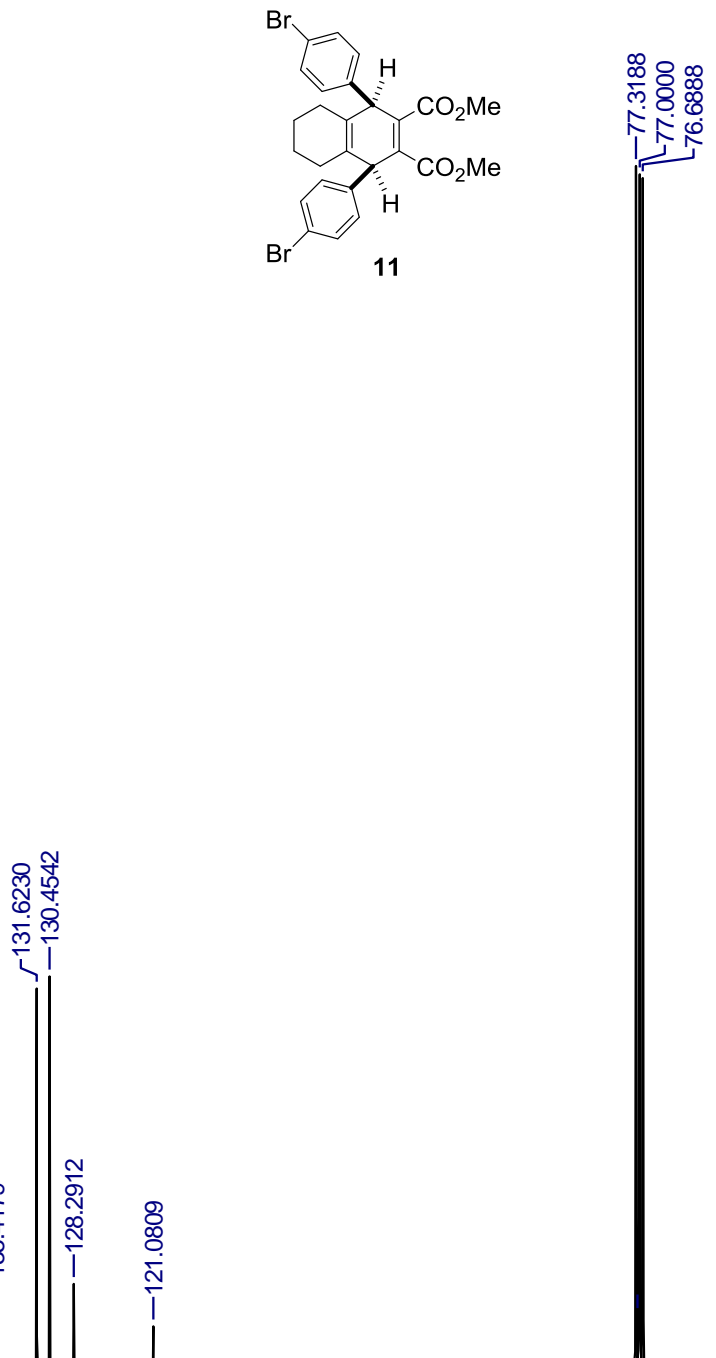

: 


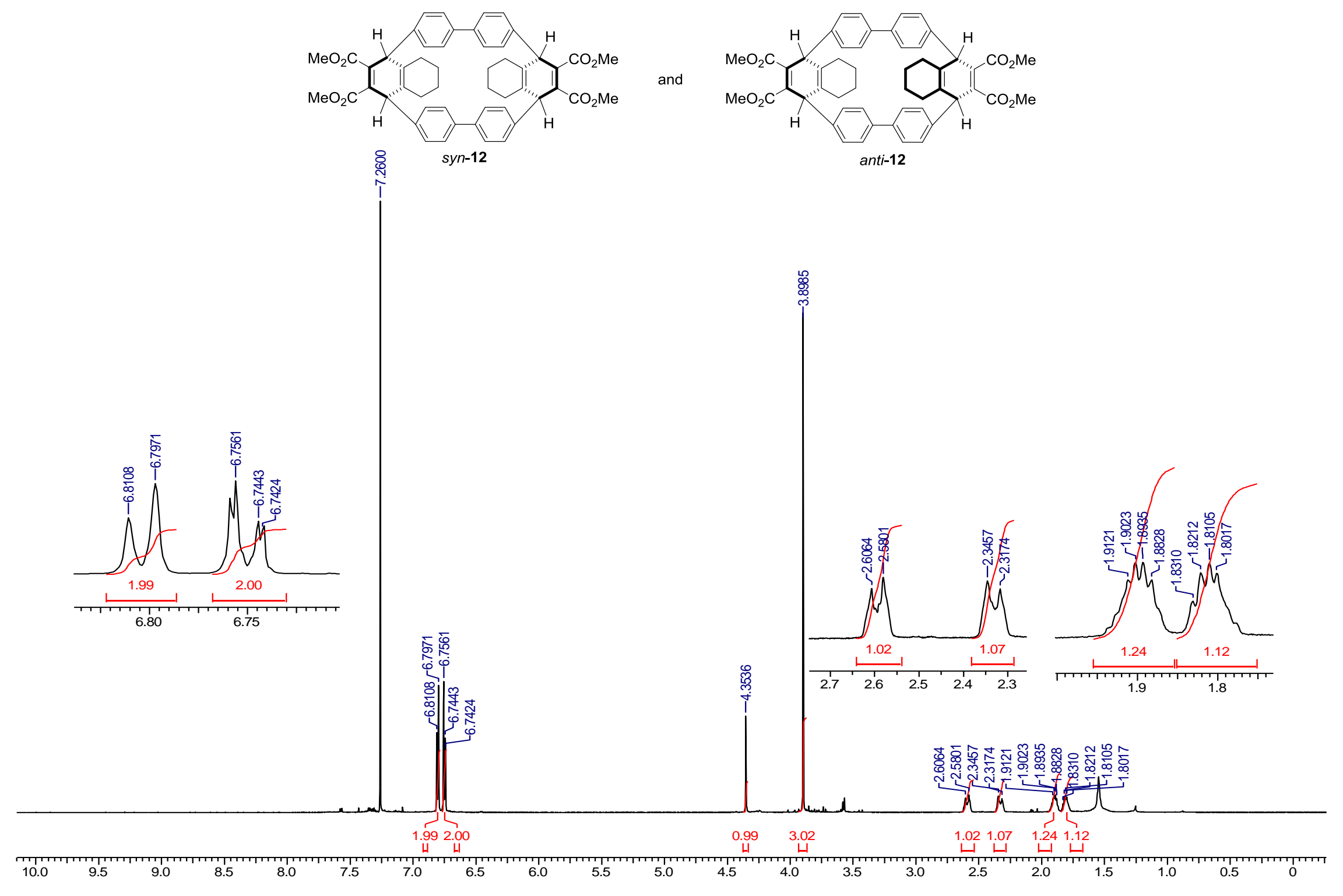




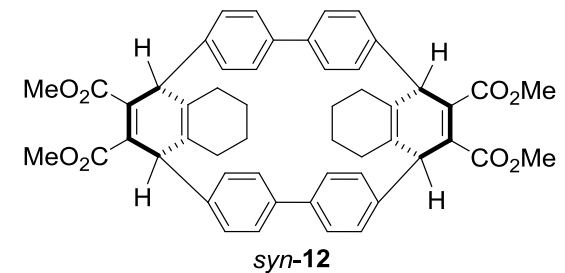

and
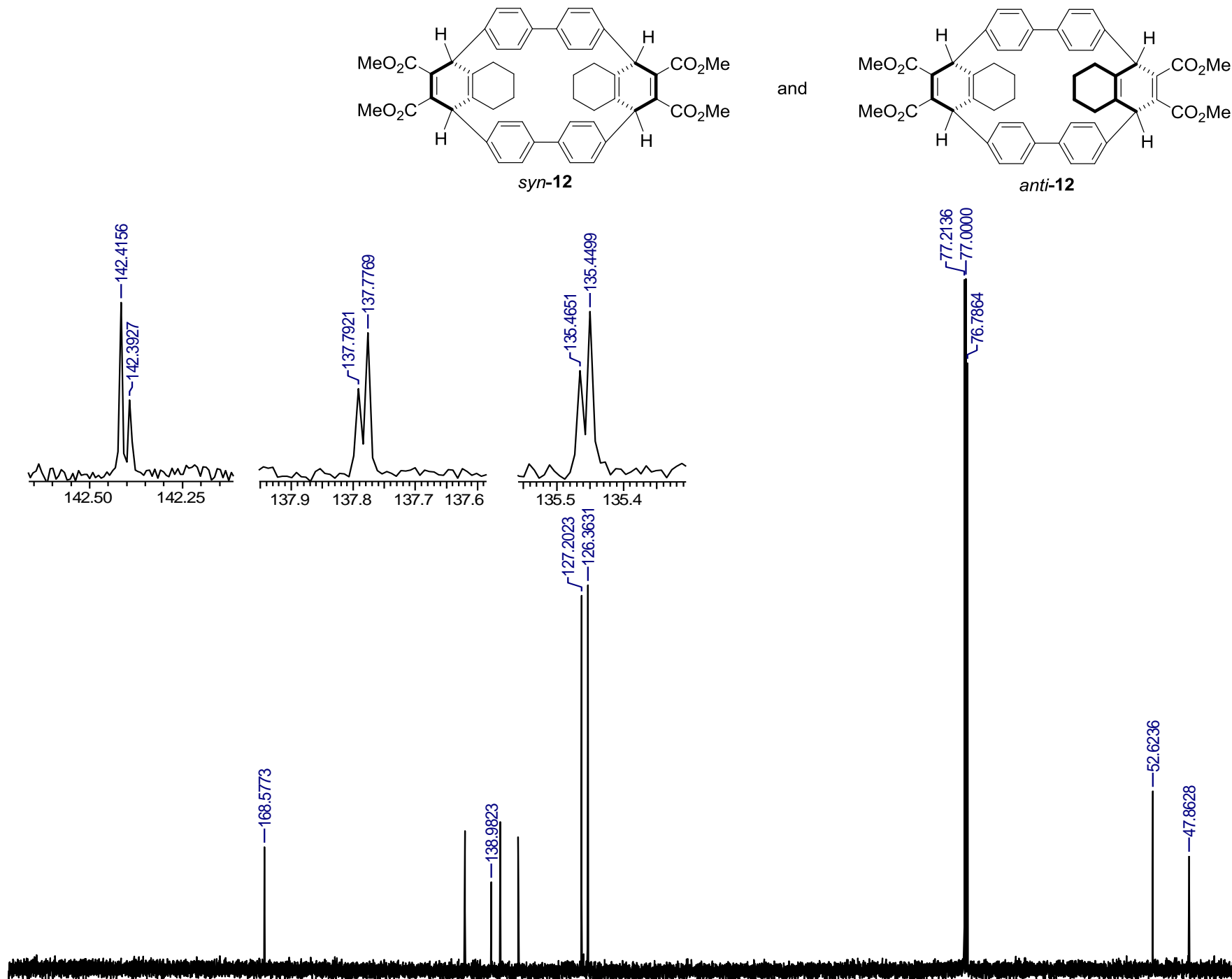

$192 \quad 18$

$184 \quad 176$

168160

1521

41361

$28 \quad 120$

$112 \quad 10$

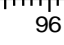

88

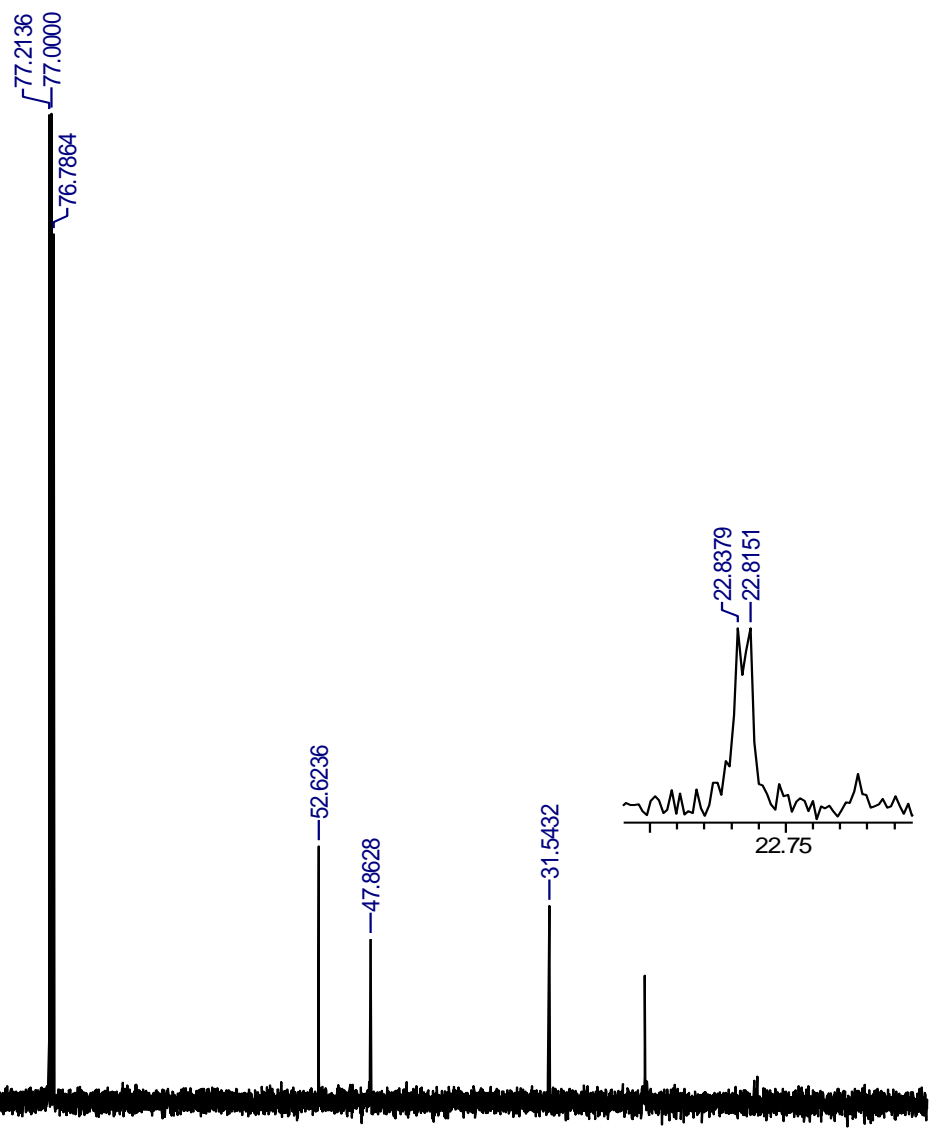

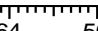

48

32

एगा1ाm 


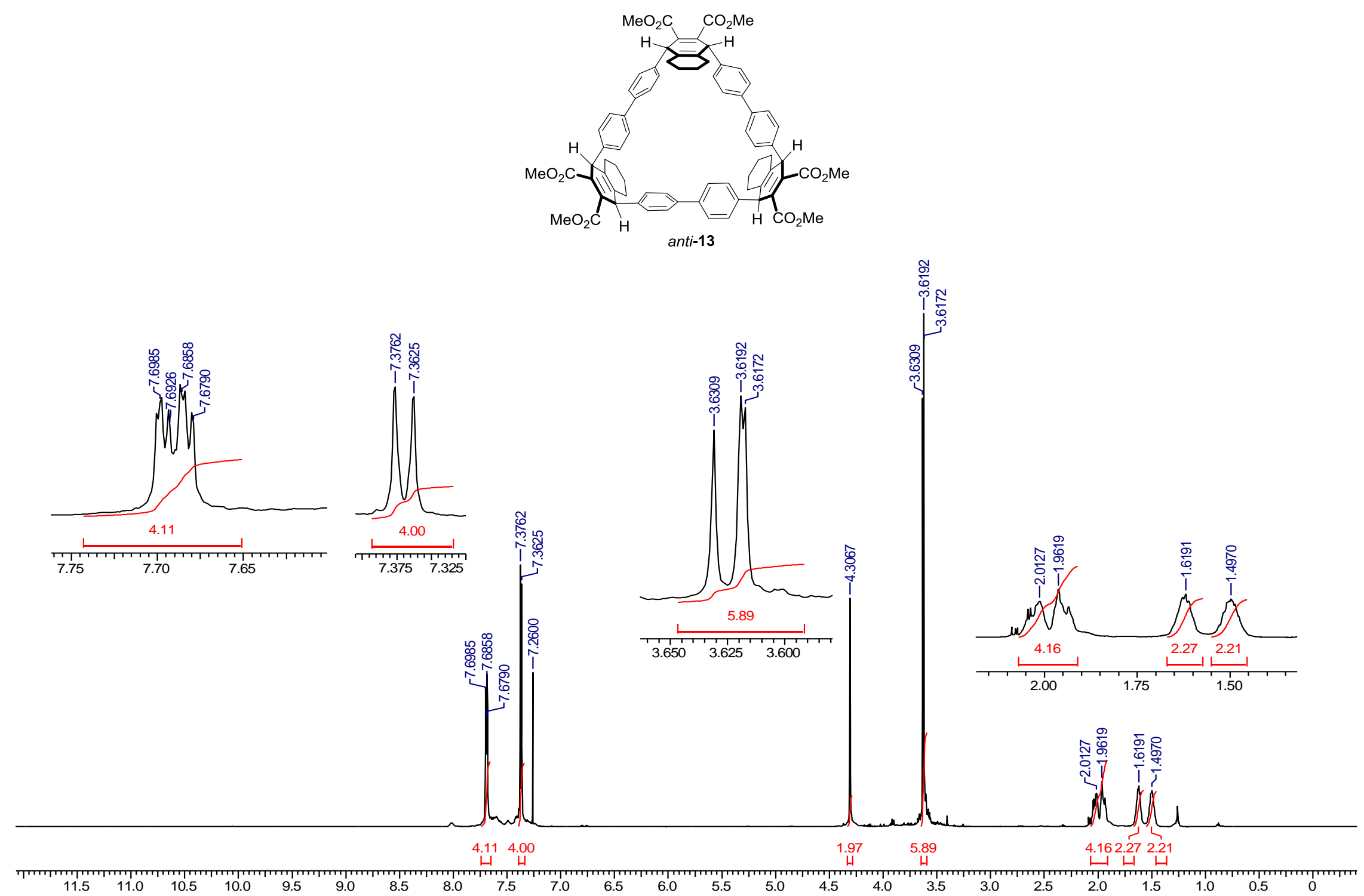




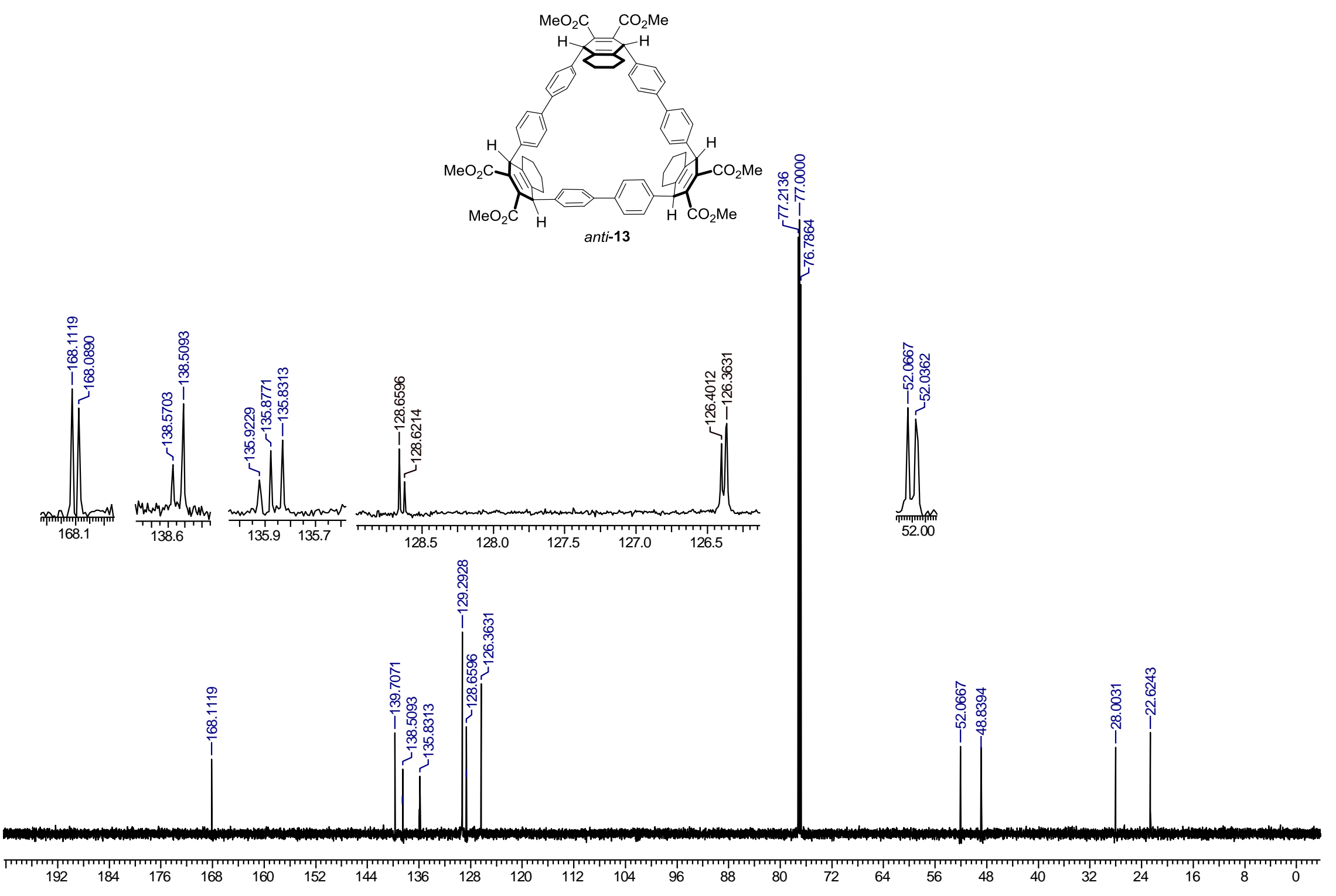




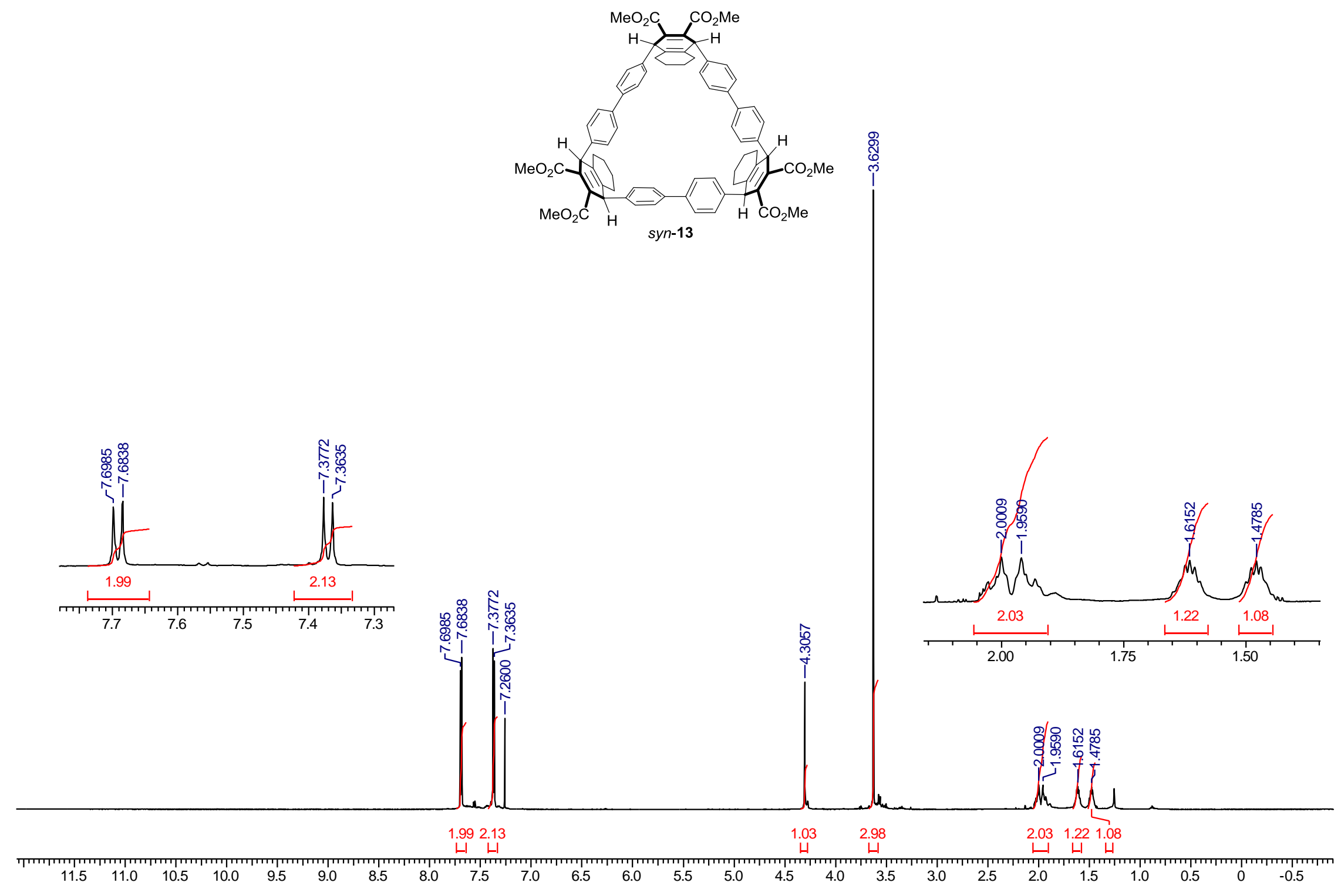



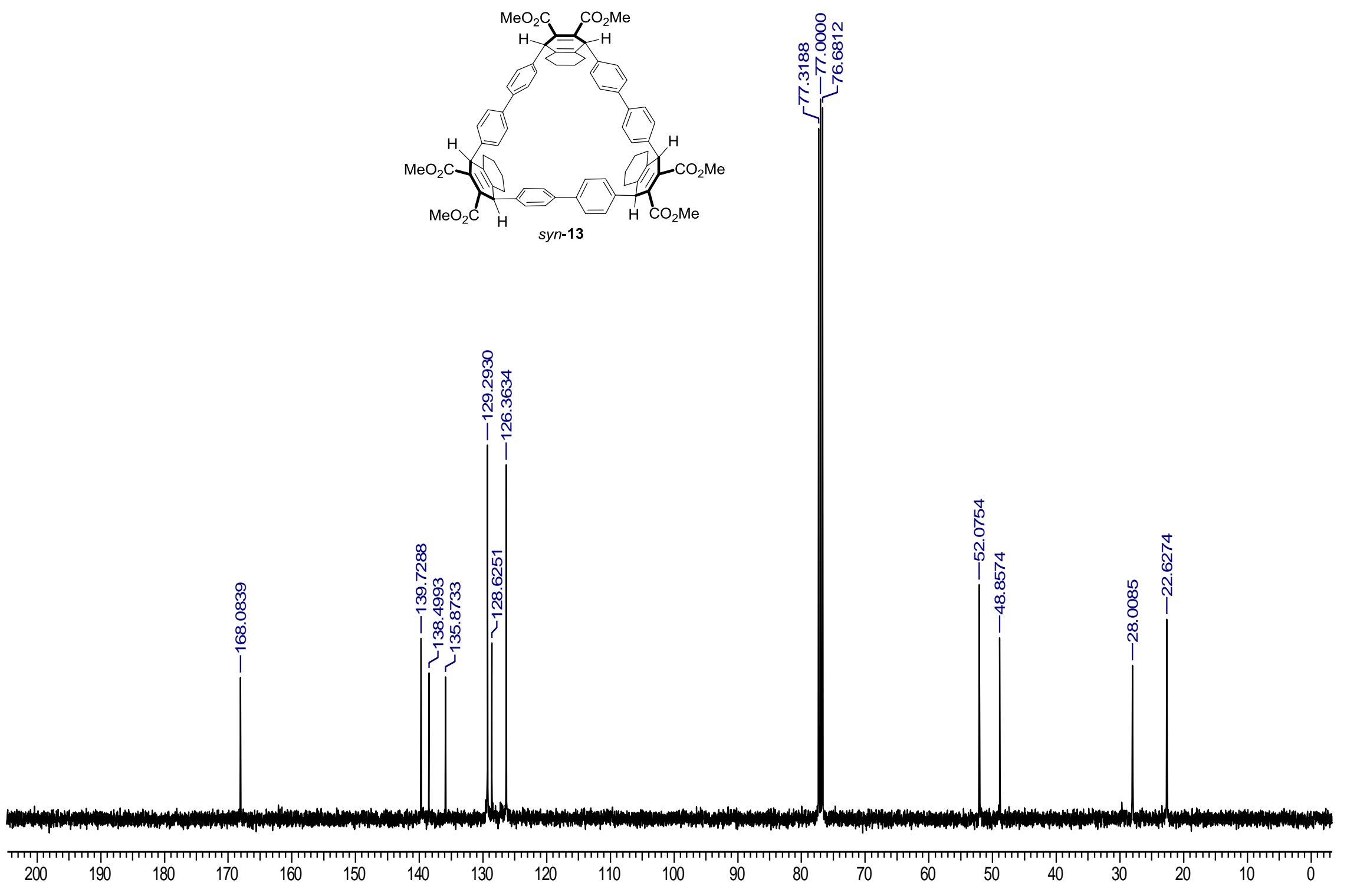


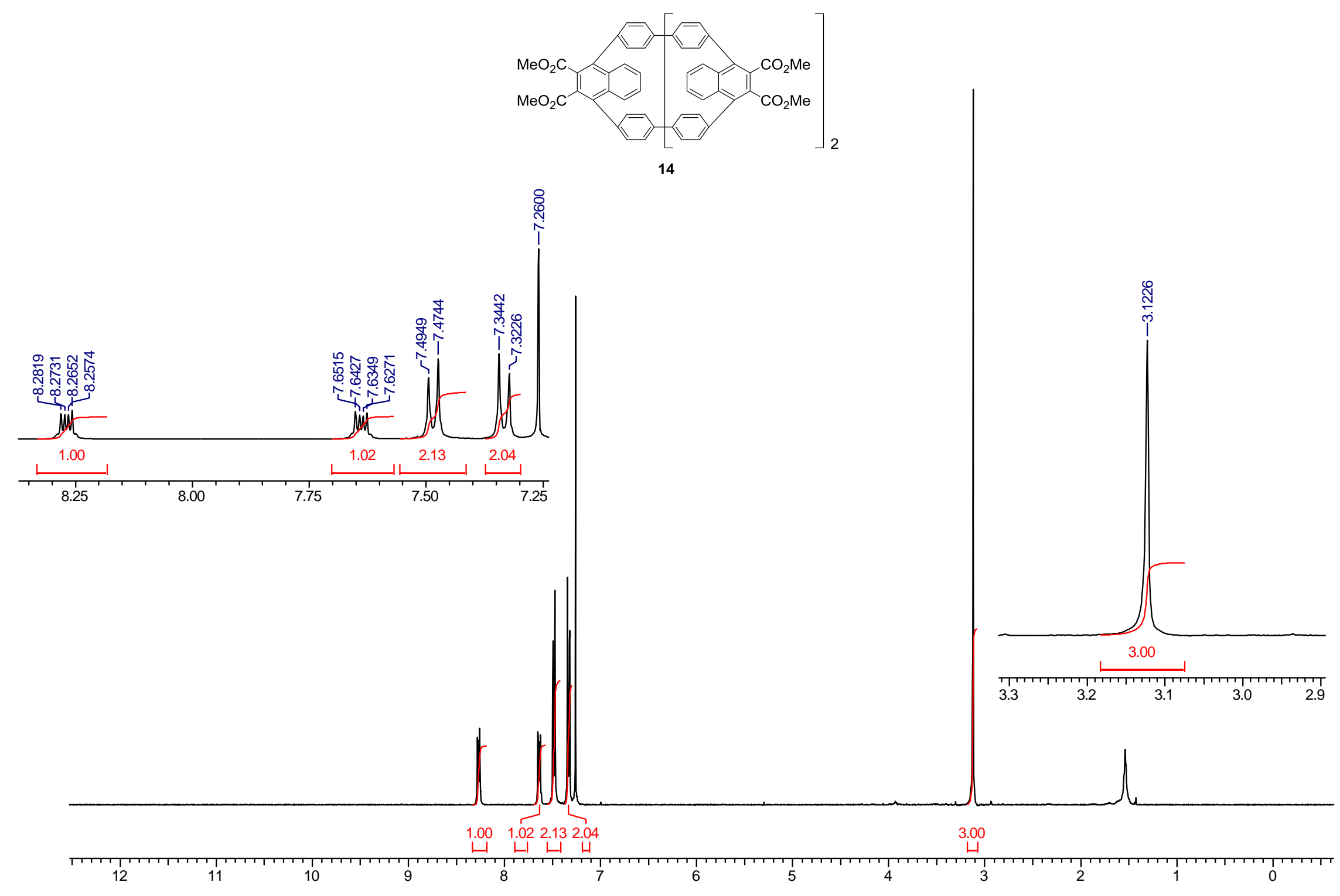




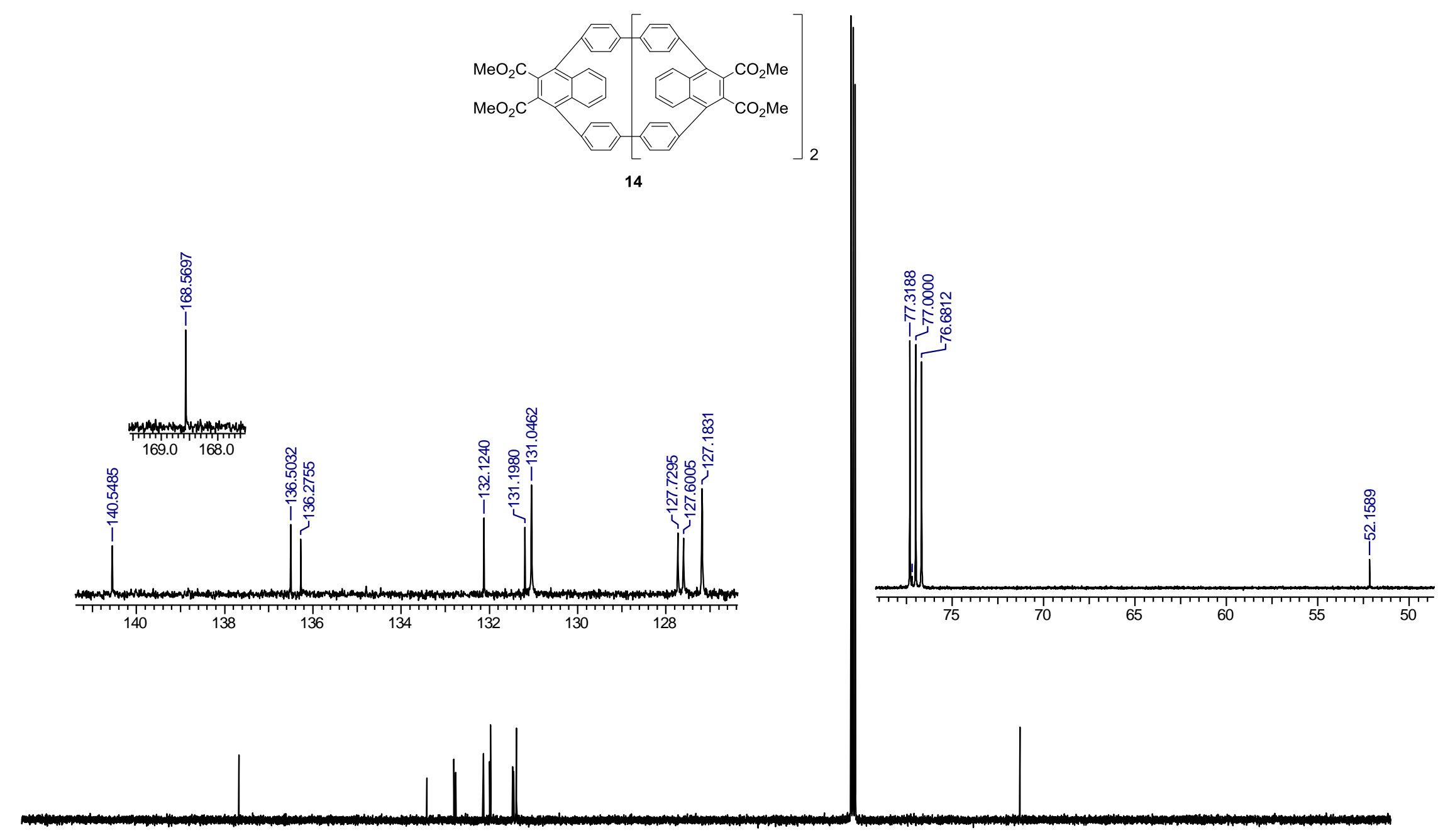

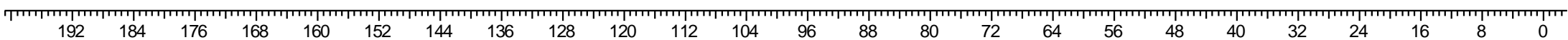

$32 \quad 24$ 


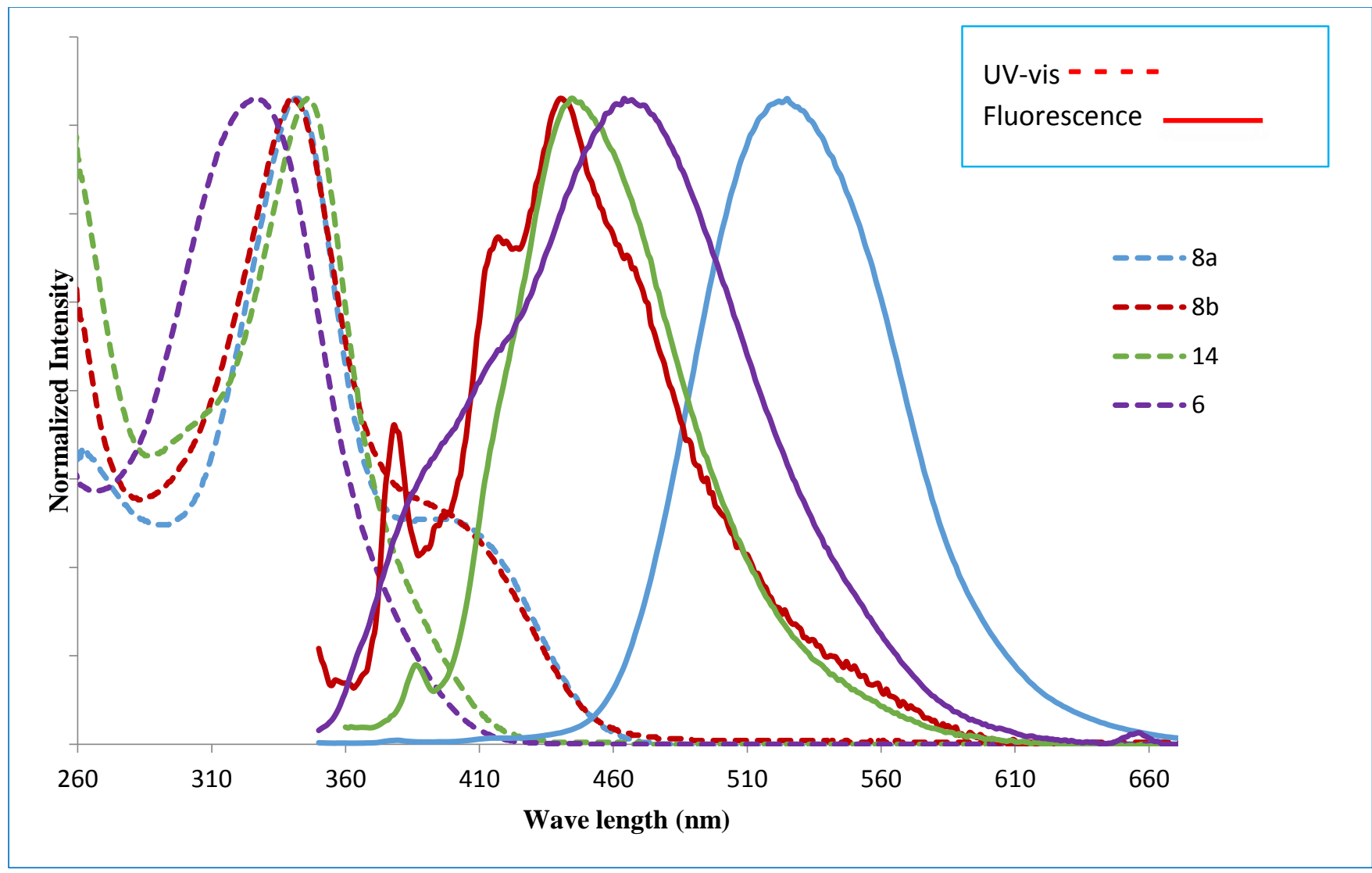

UV-vis absorption (broken line) and fluorescence spectra (solid line) of the functionalized [9]CPPs $\mathbf{6}, \mathbf{8 a}, \mathbf{8 b}$, and $\mathbf{1 4}$.

1) UV-vis absorption spectrum of 6 at the concentration of $1.4 \times 10^{-5} \mathrm{M}$ in DMSO. At the absorption maximum $\lambda_{\text {abs }}=327 \mathrm{~nm}, \varepsilon=6.7 \times 10^{4} \mathrm{~cm}^{-1} \mathrm{M}^{-1}$. Fluorescence spectrum of $\mathbf{6}$ at the concentration of $1.4 \times 10^{-5} \mathrm{M}$ in DMSO upon excitation at $327 \mathrm{~nm}$. Fluorescence $\operatorname{maximum} \lambda_{\mathrm{em}}=464 \mathrm{~nm}$.

2) UV-vis absorption spectrum of $\mathbf{8 a}$ at the concentration of $1.8 \times 10^{-5} \mathrm{M}$ in DMSO. At the absorption maximum $\lambda_{\mathrm{abs}}=342 \mathrm{~nm}, \varepsilon=2.0 \times 10^{4} \mathrm{~cm}^{-1} \mathrm{M}^{-1}$. Fluorescence spectrum of $\mathbf{8 a}$ at the concentration of $1.8 \times 10^{-5} \mathrm{M}$ in DMSO upon excitation at $342 \mathrm{~nm}$. Fluorescence $\operatorname{maximum} \lambda_{\mathrm{em}}=525 \mathrm{~nm}$. 
3) UV-vis absorption spectrum of $\mathbf{8 b}$ at the concentration of $8.6 \times 10^{-6} \mathrm{M}$ in DMSO. At the absorption maximum $\lambda_{\mathrm{abs}}=340 \mathrm{~nm}, \varepsilon=4.2 \times 10^{4} \mathrm{~cm}^{-1} \mathrm{M}^{-1}$. Fluorescence spectrum of $\mathbf{8 b}$ at the concentration of $8.6 \times 10^{-6} \mathrm{M}$ in DMSO upon excitation at $340 \mathrm{~nm}$. Fluorescence $\operatorname{maximum} \lambda_{\mathrm{em}}=440 \mathrm{~nm}$.

4) UV-vis absorption spectrum of $\mathbf{1 4}$ at the concentration of $1.7 \times 10^{-5} \mathrm{M}$ in DMSO. At the absorption maximum $\lambda_{\mathrm{abs}}=346 \mathrm{~nm}, \varepsilon=1.9 \times 10^{4} \mathrm{~cm}^{-1} \mathrm{M}^{-1}$. Fluorescence spectrum of 14 at the concentration of $1.7 \times 10^{-5} \mathrm{M}$ in DMSO upon excitation at $346 \mathrm{~nm}$. Fluorescence maximum $\lambda_{\mathrm{em}}=445 \mathrm{~nm}$. 